Portland State University

PDXScholar

Spring 5-26-2016

\title{
The Intermediate Value Theorem as a Starting Point for Inquiry-Oriented Advanced Calculus
}

Stephen Raymond Strand II

Portland State University

Follow this and additional works at: https://pdxscholar.library.pdx.edu/open_access_etds

Part of the Curriculum and Instruction Commons, and the Science and Mathematics Education Commons

Let us know how access to this document benefits you.

\section{Recommended Citation}

Strand, Stephen Raymond II, "The Intermediate Value Theorem as a Starting Point for Inquiry-Oriented Advanced Calculus" (2016). Dissertations and Theses. Paper 2914.

https://doi.org/10.15760/etd.2910

This Dissertation is brought to you for free and open access. It has been accepted for inclusion in Dissertations and Theses by an authorized administrator of PDXScholar. Please contact us if we can make this document more accessible: pdxscholar@pdx.edu. 
The Intermediate Value Theorem as a Starting Point for Inquiry-Oriented

Advanced Calculus

\title{
by
}

Stephen Raymond Strand II

A dissertation submitted in partial fulfillment of the requirements for the degree of

\author{
Doctor of Philosophy \\ in \\ Mathematics Education
}
Dissertation Committee:
Sean Larsen, Chair
Michael Oehrtman
Eva Thanheiser Joel Steele

Portland State University
2016 
(C) 2016 Stephen Raymond Strand II 


\begin{abstract}
Making the transition from calculus to advanced calculus/real analysis can be challenging for undergraduate students. Part of this challenge lies in the shift in the focus of student activity, from a focus on algorithms and computational techniques to activities focused around definitions, theorems, and proofs. The goal of Realistic Mathematics Education (RME) is to support students in making this transition by building on and formalizing their informal knowledge. There are a growing number of projects in this vein at the undergraduate level, in the areas of abstract algebra (TAAFU: Larsen, 2013; Larsen \& Lockwood, 2013), differential equations (IO-DE: Rasmussen \& Kwon, 2007), geometry (Zandieh \& Rasmussen, 2010), and linear algebra (IOLA: Wawro, et al., 2012). This project represents the first steps in a similar RME-based, inquiry-oriented instructional design project aimed at advanced calculus.

The results of this project are presented as three journal articles. In the first article I describe the development of a local instructional theory (LIT) for supporting the reinvention of formal conceptions of sequence convergence, the completeness property of the real numbers, and continuity of real functions. This LIT was inspired by Cauchy's proof of the Intermediate Value Theorem, and has been developed and refined using the instructional design heuristics of RME through the course of two teaching experiments. I found that a proof of the Intermediate Value Theorem was a powerful context for supporting the reinvention of a number of the core concepts of advanced calculus.
\end{abstract}


The second article reports on two students' reinventions of formal conceptions of sequence convergence and the completeness property of the real numbers in the context of developing a proof of the Intermediate Value Theorem (IVT). Over the course of ten, hour-long sessions I worked with two students in a clinical setting, as these students collaborated on a sequence of tasks designed to support them in producing a proof of the IVT. Along the way, these students conjectured and developed a proof of the Monotone Convergence Theorem. Through this development I found that student conceptions of completeness were based on the geometric representation of the real numbers as a number line, and that the development of formal conceptions of sequence convergence and completeness were inextricably intertwined and supported one another in powerful ways.

The third and final article takes the findings from the two aforementioned papers and translates them for use in an advanced calculus classroom. Specifically, Cauchy's proof of the Intermediate Value Theorem is used as an inspiration and touchstone for developing some of the core concepts of advanced calculus/real analysis: namely, sequence convergence, the completeness property of the real numbers, and continuous functions. These are presented as a succession of student investigations, within the context of students developing their own formal proof of the Intermediate Value Theorem. 
For my father, Stephen Strand, who taught me to be humble and generous, and for my mother, Kristie Strand, who taught me about sacrificial love and serving others. 


\section{Acknowledgements}

The achievement of this doctorate represents tireless support from numerous individuals. First and foremost, my most sincere gratitude goes to my advisor, Sean Larsen. He supported me and challenged me, and somehow did not lose faith in me, through all the growing pains of turning me into a mathematics education researcher. Through countless hours of talking, debating, data analysis, and basketball, he shaped me into the educator, thinker, and researcher that I am today. None of this would have been remotely possible without him.

I would also like to thank the other members of my dissertation committee. I thank Mike Oehrtman, who was always willing to provide a listening ear, as well as thoughtful, constructive feedback. His belief and investment in me and in my research have been invaluable. I thank Eva Thanheiser for also helping to shape and mold me into the math education researcher I am today, and for being so engaged for the final terms of my project, despite enjoying a sabbatical in Germany. And I thank Joel Steele for being so generous and flexible with his time, and for engaging so fully with this process. I could not have asked for a more positive, supportive dissertation experience, and for that I thank them all.

I am also greatly indebted to my academic siblings, Estrella Johnson and Carolyn James. All the study sessions, the feedback on work, the social outings not only helped me to be successful in our program, but also kept me sane through the process. I count them both not only as colleagues but as dear 
friends.

Of course none of this would have been possible without the students who volunteered to participate in my teaching experiments. They sacrificed a great deal of personal time and mental energy, and anything they may have learned is completely dwarfed and overshadowed by all that they taught me. My thanks also go out to Zack Reed, who manned the camera, and who was always willing to listen and to talk about math and student thinking.

I am also eternally grateful to all my friends and family. They not only put up with me, but supported me through all the trials and tribulations of a PhD program. The bottomless love and cheer I felt from each and every one of them was what carried me through.

Finally, and most importantly, I thank my loving wife and partner, Krista. The countless weekend trips, so that I could have a quiet house to myself for writing; finding and organizing endless variations of daycare, school, and babysitters over the years; being my best friend through all the ups and downs of the last seven years; for all of these, she has all my gratitude, and all my love. I count myself truly and richly blessed because of her. 


\section{Table of Contents}

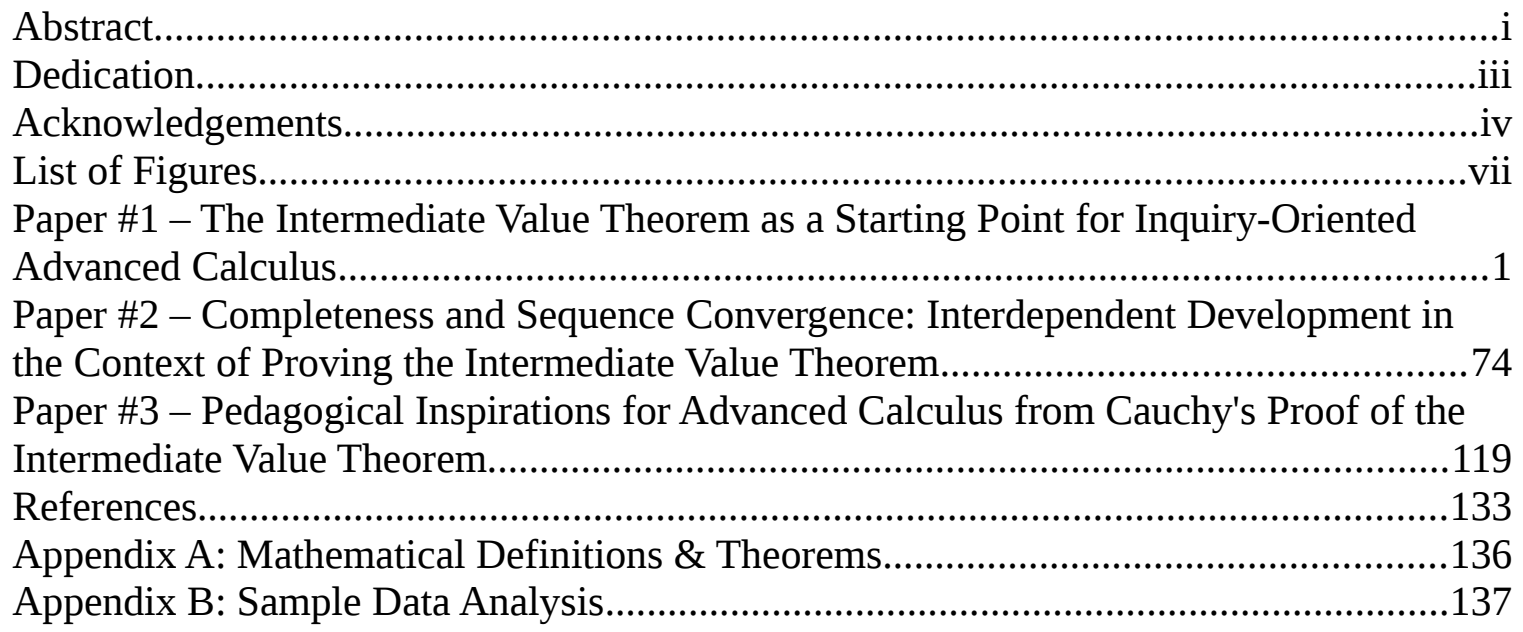




\section{List of Figures}

Figure 1: A map of the preliminary LIT...................................................................17

Figure 2: Brad and Matt's Bisection Algorithm...............................................................26

Figure 3: The first few iterations of Dylan and Jay's Decimal-Expansion Algorithm........30

Figure 4: In the highlighted expression near Matt's elbow, the root $r$ is always trapped inside this shrinking interval, between the endpoints an and bn, the distance between which also bounds the error in their approximation at the nth step.

Figure 5: The MCT, with Dylan and Jay's added characterization of $b$ as the least-upper bound

Figure 6: Jay's sketch of the damped sine curve, which he and Dylan used as their prototypical example of convergence.

Figure 7: Dylan and Jay's formal definition of sequence convergence..............................43

Figure 8: Dylan and Jay's criteria for a sequence to "decrease to zero"..............................44

Figure 9: Dylan's sketch arguing why the least-upper bound of the sequence must exist. 47

Figure 10: Dylan and Jay's first attempt at a formal definition of "continuous"................55

Figure 11: The first three iterations of Dylan and Jay's Decimal-Expansion algorithm....85 Figure 12: Dylan's justification for the convergence of their sequence of approximations.

Figure 13: Dylan and Jay's first attempt at defining sequence convergence......................92

Figure 14: Dylan and Jay's first proof of a modified MCT..................................................96

Figure 15: The students' argument reproduced for legibility..............................................96

Figure 16: Students' definition of a sequence "decreasing to zero"....................................98

Figure 17: Jay's first attempt at defining "maximum"......................................................100

Figure 18: Dylan and Jay's refined definition of the "maximum" of a sequence.............102

Figure 19: Statement of the students' conjectured MCT....................................................104

Figure 20: Jay's string of inequalities showing that the ei's were decreasing...................105

Figure 21: Jay's string of inequalities reproduced for legibility.........................................105

Figure 22: A damped oscillating sequence....................................................................108

Figure 23: The sketch supporting Dylan's argument that they could assume the existence

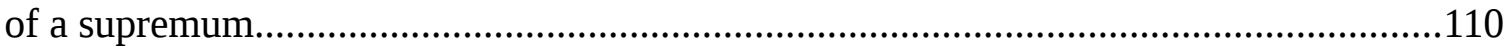

Figure 24: The Intermediate Value Theorem for continuous functions...........................121

Figure 25: A student's brief statement of their algorithm using the Bisection Method.. .124

Figure 26: A few sample iterations of students' Decimal Expansion algorithm for

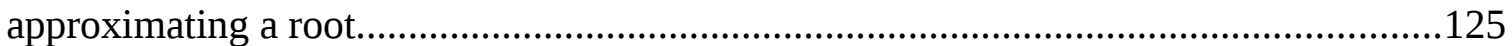

Figure 27: Students discussing how to prove their conjecture (which is essentially the MCT)

Figure 28: Students recall the limit characterization of continuity from calculus............130 


\section{Paper \#1 - The Intermediate Value Theorem as a Starting Point for Inquiry- Oriented Advanced Calculus}

Abstract: In recent years there has been a growing number of projects aimed at utilizing the instructional design theory of Realistic Mathematics Education (RME) at the undergraduate level (e.g., TAAFU, IO-DE, IOLA). This project represents the first steps in such an instructional design effort aimed at advanced calculus. In this paper I describe the development of a local instructional theory (LIT) for supporting the reinvention of formal conceptions of sequence convergence, the completeness property of the real numbers, and continuity of real functions. This LIT was inspired by Cauchy's proof of the Intermediate Value Theorem, and has been developed and refined using the instructional design heuristics of RME through the course of two teaching experiments. I found that a proof of the Intermediate Value Theorem was a powerful context for supporting the reinvention of a number of the core concepts of advanced calculus.

\section{Introduction}

Making the transition from calculus to advanced calculus/real analysis can be challenging for undergraduate students. Part of this challenge lies in the shift in the focus of student activity, from a focus on algorithms and computational techniques to activities focused around definitions, theorems, and proofs. The goal of Realistic Mathematics Education (RME) is to support students in making this transition by building on and formalizing their informal knowledge. There are 
a growing number of projects in this vein at the undergraduate level, in the areas of abstract algebra (TAAFU: Larsen, 2013; Larsen \& Lockwood, 2013), differential equations (IO-DE: Rasmussen \& Kwon, 2007), geometry (Zandieh \& Rasmussen, 2010), and linear algebra (IOLA: Wawro, et al., 2012). This project represents the first steps in a similar RME-based, inquiry-oriented instructional design project aimed at advanced calculus.

To begin this instructional design project, what I needed to find was a starting point that was experientially real for the students; that is, a context in which students could reason intuitively, using the intuition, skills, and knowledge developed during the calculus sequence. Further, ideally this context would be rich in some of the core concepts of advanced calculus. I will demonstrate how the proof of the Intermediate Value Theorem is just such a context.

In his Cours d'Analysis, Cauchy presented one of the first formal proofs of the Intermediate Value Theorem (IVT) for continuous functions (Grabiner, 1981). This is remarkable, at least in part, because prior to this proof many mathematicians had either taken the IVT as a definition of continuity, or as so obvious as not to require proof. Not only was the proof's very existence novel, in it Cauchy utilized a novel proof technique: turning a process of approximation of a root into an argument for the existence of a root.

While the theorem itself is intuitive with a basic understanding of functions and real numbers, the proof requires a fairly sophisticated understanding of convergence, continuity and functions, as well as the completeness of the real 
numbers ${ }^{1}$. These features taken together lead me to hypothesize that, for postcalculus students, the development of a proof of the Intermediate Value Theorem could serve as a useful context from which to develop more formal understandings of sequence convergence, continuity, and completeness.

Using the instructional design heuristics of RME and a design research approach, this project represents the initial stages in the development of a local instructional theory and instructional sequence, using the Intermediate Value Theorem as a starting point and touchstone for advanced calculus. Gravemeijer described design research as being, "formed by classroom teaching experiments that center on the development of instructional sequences and the local instructional theories that underpin them" (2004, p. 108). In design research, theory and instructional practice are developed in tandem, in a reciprocal fashion (Cobb, et. al., 2003). Through the lens of RME (Gravemeijer, 1998), a local instructional theory should provide a theoretical explanation for how students might be supported in reinventing the desired mathematics. Such a theory can be thought of as an idealized instructional sequence, where the design heuristics of RME are used to explain student activity and development. Furthermore, such a theory should provide the framework for an instructional sequence. As Larsen explains, "The primary purpose of a local instructional theory is to support the design of an instructional sequence that is appropriate for a given instructional context" (2013).

1 Even Cauchy did not explicitly acknowledge the completeness of the real numbers in many of his early proofs (Grabiner, 1981; Lützen, 2003). 
Below I propose a local instructional theory for the development of the concepts of convergence of sequences, the completeness of the real numbers, and continuity, using the proof of the IVT as a starting point and touchstone. The questions that guided the development of this local instructional theory (as well as the analysis of student activity) are adapted from Gravemeijer (1998):

1. What student strategies anticipate formal mathematical topics in advanced calculus?

2. What tasks or problems elicit these strategies?

3. How can these strategies be leveraged to support the development of formal mathematical topics in advanced calculus?

After discussing the relevant literature, as well as the theoretical tools I used in this design research, I will describe the development of this LIT. This description will begin with a preliminary LIT, that I developed before the teaching experiments and that I used to design the instructional sequence for those teaching experiments. I will then present data from the teaching experiments, illustrating how that data informed the refinement of the LIT. Finally, I will present the Proposed LIT.

\section{Literature Review}

Central to developing a local instructional theory (LIT) is an understanding not only of student thinking about the core concepts, but also how that thinking might grow and develop in formality. In this section I will describe the current state of knowledge of student thinking on the core concepts for this LIT: namely, 
limits/convergence, completeness, and continuity.

Student understanding of limit has received a great deal of attention from the mathematics education research community. Much of this research has focused on investigating the struggles students face in working with limits and the tools they use to deal with those struggles (Bezuidenhout, 2001; Cornu, 1991; Davis \& Vinner, 1986; Moru, 2009; Oehrtman, 2009; Sierpińksa, 1987; Szydlik, 2000; Tall \& Schwarzenberger, 1978). The other main area of focus has been investigating the process of students formalizing their understanding of limit (Cottrill, et al., 1996; Oehrtman, Swinyard, \& Martin, 2014; Swinyard \& Larsen, 2012; Williams, 1991); that is, coming to understand and work with limits in a way that is consistent with the standard formal definition(s).

Through the calculus sequence students' primary activity with limits involves finding limits. Swinyard \& Larsen (2012) observed that a formal definition of limit is not useful in this context; rather, the formal definition is useful for verifying limit candidates. This observation suggested that, not only does student thinking about limit progress in formality, the activity has a fundamentally different nature at the formal level. Motivating this shift in character, while still building on intuitive knowledge gained in the calculus sequence, heavily influenced the development of the local instructional theory and task sequence for this design experiment. Further, this work contributes to our understanding of how student thinking with regards to limits progresses in formality, especially in the context of developing proofs with limits. 
Because there is no current research dealing directly with student thinking about completeness, I turned to a historical work for insight into how the mathematics community's thinking about completeness developed. In what is possibly the first explicit treatment of the topic of completeness, Dedekind (1901) decries the fact that the mathematics community of the day seemed to take the completeness property of the real numbers as an obvious consequence of the geometric representation of the real numbers as a number line. This was unsatisfactory because so many important results in real analysis (including the IVT) depended on this property; Dedekind argued that such an important concept needed an algebraic justification. In that work Dedekind laid out an algebraic construction of the real numbers from the rational numbers, in order to prove, without appealing to geometry, that the real numbers really were complete in the sense that real analysis needed them to be. Dedekind's work suggests that students will likely take the completeness of the real numbers as a natural consequence of their representation as a number line, just as mathematicians did historically. This study will contribute to our understanding of how student thinking about completeness might develop.

Tall \& Vinner (1981) identified some common informal conceptions of continuity in calculus students. When shown graphs of several different functions, students were asked to determine whether each function was continuous. Student justifications fell into three general categories: the graph was all in one piece, the function was given by a single formula, or there were no sudden 
changes in gradient (the graph was smooth). The first justification was the most common, and was the least problematic (for functions defined on an interval this is essentially correct). However, Tall \& Vinner's study demonstrates one potential source of difficulty identified by Jayakody \& Zazkis: there is no standard definition of a "continuous function". Further, even limit characterizations presented in calculus textbooks can lead to conceptual conflict, as some books suggest a function is discontinuous if it is undefined (e.g., $1 / x$ at $x=0$ ). This is inconsistent with the standard formal characterization of continuity, which only applies to points within a function's domain. This research suggests that students will likely come out of the calculus sequence thinking of continuity in terms of an unbroken curve or in terms of the limit characterization (which comes with its own difficulties), depending on the context. Unfortunately, little is known about how student thinking about continuity might develop. This study, and future studies in this instructional design project, will shed light on the development of student understanding of continuity.

I will now describe the theoretical constructs that supported the design and implementation of this design research project, as well as the development and refinement of the LIT.

\section{Theoretical Framework}

The local instructional theory presented in this paper was developed using 
the instructional design heuristics of RME and refined through the implementation and analysis of two teaching experiments. Specifically, I drew on the design heuristics of guided reinvention, emergent models, and didactic phenomenology. I will provide a brief explanation of each of these heuristics below.

\section{Guided Reinvention}

On a macro level, the heuristic of guided reinvention motivated my overall instructional goal of having the students develop their own formal definitions of convergence, rather than working to make sense of the standard formal definitions. In RME, the goal is not that everything be strictly reinvented by the students, but rather that, "formal mathematics would be experienced as an extension of [students'] own authentic experience" (Gravemeijer \& Doorman, 1999). That is, instructional activities should be designed so that the formal mathematics emerges from students' informal mathematical activities, so that students feel a sense of ownership over the mathematics developed. While guided reinvention provides a macro-level structure for instructional design, other RME heuristics are more useful at filling in this structure. For actual task generation, sequencing, and refinement, I relied largely on the design heuristics of didactic phenomenology and emergent models. 


\section{Didactic Phenomenology}

As with all RME design heuristics, didactic phenomenology has been shaped and continues to be shaped by its use in research. Usiskin (1985) described phenomenology as the connections between mathematical structures and the complex reality which they were created to model. Thus didactic phenomenology refers to the consequences of these connections for instruction. Freudenthal (1983) argued that understanding the phenomenology of a mathematical topic was vital to both teaching and understanding that topic. Put another way, in order to engage students with a mathematical topic, it is essential to investigate and take lessons from the historical development of said topic. What problems did this topic solve, and how did it solve them? How can these problems be made accessible to students?

Of course, undergraduate students do not have the same knowledge, skills, or concerns as historical mathematicians. Didactic phenomenology can still be useful even when historical problems are not accessible to students. Gravemeijer and Terwel abstracted these principles, suggesting that, "situations should be selected in such a way that they can be organized by the mathematical objects which the students are supposed to construct" (2000, p. 787). This is the essence of didactic phenomenology: if we want students to develop certain tools, we need to provide them with problem contexts whose solution requires those 
tools, or in which those tools have the power to help the students solve meaningful problems. In this way didactic phenomenology can be a powerful tool for instructional design.

This suggests that, in order to support students in reinventing formal definitions of sequence convergence and continuity, students should be presented with contexts and tasks in which they would able to reason intuitively, and in which those formal definitions would have power to organize and solve problems for them. Inspired by the works of Cauchy and Bolzano, I conjectured that approximating the roots of a polynomial using the Intermediate Value Theorem (IVT), and then constructing a formal proof of the theorem ${ }^{2}$, would be just such a context.

\section{Emergent Models}

The heuristic of emergent models provides one way to describe the process by which formal mathematics might emerge from informal student activity. The use of "models" in RME is not restricted to physical drawings or tools. In describing a local instructional theory for the development of the quotient group concept, Larsen conjectured that, "the quotient group concept could emerge as a model-of students' informal mathematical activity as they searched for parity in the group D8 (the symmetries of a square)" (Larsen \& Lockwood,

2 Technically, if we restrict ourselves to establishing the existence of roots of continuous functions, then we are only proving a special case of the IVT (sometimes referred to as Bolzano's Theorem). But the proof is easily adapted to the general case by a simple vertical shift. 
2013). Thus "model" in this sense can also refer to a concept or structure that the teacher or researcher recognizes as a model of the students' mathematical activity, but of which the students themselves may not be aware. Continuing with Larsen's example, once the students had begun to reflect on their activity with parity and other group-like partitions of groups, conjecturing and verifying common properties, the concept of quotient group became a model for their reasoning in this new mathematical reality; a model-of informal mathematical activity had become a model-for more formal mathematical reasoning. "This shift from model of to model for concurs with a shift in the students' thinking, from thinking about the modeled context situation to a focus on mathematical relations" (Gravemeijer, 1999, p. 162).

Another way to characterize the development of an emergent model is with levels of activity (Gravemeijer, 1999). Initial student activity begins at the situational level, and is primarily composed of organizing a given problem context. In RME this is called horizontal mathematizing. At this level the model emerges as a model-of student thinking. Activity transitions to the referential level when students refer back to their previous activity, reflecting on and organizing that activity. This focus on mathematical relations, the reflective practice of organizing their own activity, is referred to as vertical mathematizing (Gravemeijer, 1999). This then becomes a new, more formal level for horizontal mathematizing. In RME-based instruction, this progressive mathematization is the primary mechanism by which students develop more formal mathematics and 
create new mathematical realities for themselves. The model transitions to a model-for more formal reasoning as student activity transitions to the general level. At this level students may no longer need to refer back to the original context, and are able to use the model to reason in a new context.

While the principle of guided reinvention motivates the over-arching goals of this local instructional theory, the heuristics of emergent models and didactic phenomenology were more instrumental in actual task design, sequencing, and refinement. Didactic phenomenology suggested that I find contexts in which the students could reason intuitively and which required the tools I wished them to develop. Further, this heuristic suggested that I look to the origins of the formal definition of limit for inspiration. These led me to choose the context of approximating roots with the IVT, and adapting those strategies to a proof of said theorem. The heuristic of emergent models suggested I find a model (or models) that could characterize student activity and support students in progressive mathematization. At least two useful models emerged from student activity in the context of approximating roots with the IVT, and in turn became powerful models for more formal mathematical reasoning in the construction of a proof of the IVT. In these ways the design heuristics of RME guided the development of this LIT.

\section{Pedagogical Content Tools}

While the design theory of RME offers heuristics for instructional design, it 
does not explicitly offer tools for teachers implementing such instructional sequences. Rasmussen \& Marrongelle (2006) described some specific activities in which teachers can engage in the classroom that support student learning in ways that are consistent with RME. Of particular interest for this study will be the transformational record. The authors give two criteria for a teacher move to be a transformational record: "(1) some form of notation (typically informal or unconventional notation) was either used by a student in whole-class discussion or introduced by the teacher to record or notate student reasoning and (2) this notational record was then used by students in achieving subsequent mathematical goals" (p. 394).

With these constructs, I will now describe my preliminary attempt at a local instructional theory, which focused on the concepts of limits and continuity.

\section{Preliminary Local Instructional Theory}

In this section I will present an overview of my early efforts at a local instructional theory for the development of some of the core concepts of advanced calculus. The concept of limit has served as the theoretical foundation for the calculus and its applications ever since the work of Cauchy, Bolzano, and others in the early and mid 19th century (Grabiner, 1981). It follows that a formal understanding of the limit concept is essential to any investigation of the theoretical underpinnings of the calculus, and for this reason much of my early 
effort was spent on this concept. With this project I sought to put the instructional design heuristics of RME to use in translating what we as a field knew with regard to student thinking about limit (and specifically about formalizing the limit concept) into an instructional sequence, the goal of which was to support students in developing formal definitions of different types of convergence (e.g., sequence convergence, and function limits at infinity and at a point, etc.). In accordance with didactic phenomenology, I sought contexts and problems whose solution would require such formal definitions. Inspired by Cauchy's proof and my own mathematical concept analysis, I hypothesized that a proof of the Intermediate Value Theorem, built on adapting approximation techniques, could be just such a problem. In trying to construct a formal proof, students would need formal definitions of sequence convergence and of continuity, and additionally they might need formal definitions related to the limit of a function at a point. More specifically, they would need to justify why their approximations would converge to a root of the polynomial (or more generally, the root of a continuous function), and why continuity was necessary for this process to work. In this way formal definitions would be born from a specific need, to be used for a specific purpose (constructing a formal proof).

The design heuristics of RME guided this local instructional theory on two levels. On a macro level, the heuristic of guided reinvention motivated my instructional goal of having the students develop their own formal definitions, rather than working to make sense of standard formal definitions. The heuristic of 
didactic phenomenology suggested that in order to have students reinvent such formal definitions, I needed to find contexts and tasks in which the students were able to reason intuitively, and which required such formal definitions in order to be successful. Because of the centrality of the limit concept in advanced calculus, my early efforts focused primarily on that concept.

The heuristic of emergent models provided a possible path for this development in the following way: the development of the concept of limit within the mathematics community in the 18th and 19th centuries suggests that the algebra of approximations could emerge as a model-of student activity. This idea is explored in more depth below, but briefly: history suggests that building from students' intuitions about approximation and error-bounding could support students in formalizing their understanding of limit. Oehrtman's research into curriculum design for introductory calculus gives further support to this idea (2008).

The algebra of approximations played a pivotal role in the historical development of the limit concept (Grabiner, 1981). By "the algebra of approximations" I refer collectively to the mathematical tools of approximation: inequality and absolute-value expressions, along with the algebra of operating with these expressions ${ }^{3}$. Mathematicians (and in particular Lagrange) of the late 18th and early 19th centuries had made great strides in techniques of

3 It should be understood that geometric and graphical reasoning will be an important part of this model as well. However, as analysis proofs generally rely primarily on algebraic representations for formal levels of rigor, students' geometric and graphical reasoning will be leveraged to inform and support the development of algebraic tools and skills. 
approximation and error-bounding in applied contexts (Grabiner, 1981). One of Cauchy's great insights and contributions was to re-purpose these approximation techniques into techniques for proving existence. In this way these approximation techniques became a model-for constructing formal proofs of key calculus concepts, including the IVT (Grabiner, 1981). Thus the algebra of approximations could be viewed as an emergent model in the historical formalization of calculus. I hypothesized that, in guiding students to reinvent formal conceptions of limit and of continuity, the algebra of approximations could serve as an emergent model in student activity as well. Building on students' intuitions about approximations and error-bounding has met with some measure of success as a starting point and unifying theme for introductory calculus (Oehrtman, 2008), and my hope was that it would also support students in the more formal endeavors of advanced calculus.

In Figure 1, I have put together a map of the macro-scale of my local instructional theory. The boxes represent horizontal mathematizing on the part of the students. The vertical arrows indicate vertical mathematizing, where the students reflect on and organize their own mathematical activity, thereby creating new mathematical realities for themselves. The proof of the IVT very naturally involves three parts: an exploratory part, in which the IVT is conjectured, and tools for its proof (e.g., an approximation algorithm) are developed; a deductive part focused on the inputs of the function, in which one uses the approximation algorithm to construct a sequence which converges to a root-candidate (and 
justifies this convergence); and finally, a further deductive part coordinating the inputs and outputs of the function, in which one proves that the continuity of the function guarantees that this root-candidate really is a root. This preliminary LIT is built around this structure.

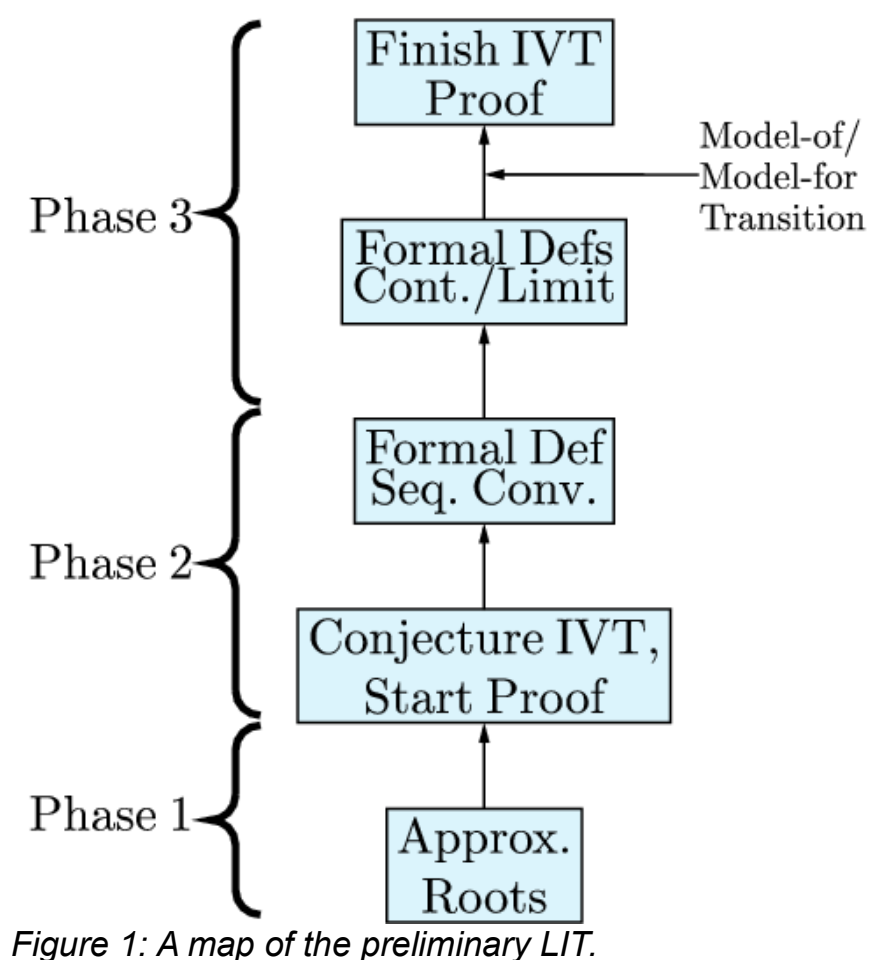

The first set of tasks (Phase 1, Figure 1) involves students approximating roots to a polynomial, using the idea of the IVT. The algorithm they construct will provide a sequence as the object of study in Phase 2. The over-arching task of Phases 2 and 3 is proving their strategy for finding the root will work for any continuous function that changes sign on an interval (the IVT). In order to 
complete this proof, it will be necessary to have formal definitions of sequence convergence (for the inputs), continuity (which will like be built on the limit of a function at a point). Thus Phase 2 involves reflecting on the activity of Phase 1 to construct a formal definition of sequence convergence. This will allow the students to justify that their algorithm gives them a root-candidate (under the assumption that the root exists). Then in Phase 3 students work to capture the ideas of continuity and limit with definitions, so that a satisfactory proof can be completed. It is Phase 3 in which the desired development of a formal understanding of the limit concept occurs, as evidenced by the construction of a formal definition of limit. This also results in a formal definition of continuity (through the limit characterization from calculus). The algebra of approximations (now manifested as a formal definition of limit/continuity) then becomes a modelfor more formal reasoning as students use it to complete the proof of the IVT.

In keeping with the principles of design research, this LIT has been refined through the course of two teaching experiments. Below I describe the structure of the two teaching experiments, as well as the manner in which I analyzed the data. In the Results section I will detail the activity of the students through the course of the two teaching experiments, highlighting how my analysis informed the refinement of the LIT. Finally, I will present my current LIT for the development of the concepts of sequence convergence, completeness, and continuity. 


\section{Methods}

As a part of the instructional design process, I ran two separate teaching experiments over the course of a year, a little more than six months apart. Each teaching experiment consisted of 10 , hour-long sessions with myself as teacher/researcher ${ }^{4}$ and a pair of students working at a chalkboard at the front of the room. These students were volunteers selected from courses that were direct prerequisites to advanced calculus/elementary real analysis courses (e.g. Linear Algebra, Discrete Mathematics, Abstract Algebra, and Introduction to Proof), and who had expressed an intention to take advanced calculus in the near future. All four participants had completed the calculus sequence, differential equations, and at least one proofs-based course, prior to participating in the teaching experiment. I will call the students from the first teaching experiment Brad and Matt, while the students from the second teaching experiment are here called Dylan and Jay.

My primary goal during ongoing analysis was to understand how students were thinking about the tasks in which they were engaging, as well as how they were thinking about the strategies they were employing. For this study I was particularly interested in how the concept of completeness was present in student thinking and justifications, and how that thinking might be leveraged and developed. To aid in this, I wrote session summaries and I kept a spreadsheet for each session, recording general student activities over the course of the session

4 For the first teaching experiment I also had a graduate student operating the video camera. 
and marking segments for later transcription.

During the implementation of the teaching experiments, there were anywhere from three days to an entire week between sessions. During that time I watched the videos of the previous session, creating written session summaries, and tried to identify student statements and strategies that begged for further investigation. For example, in the first session, while Brad and Matt were generating approximations to the root of a polynomial, they made statements about how "good" their approximations were. During that session I was focused on other things, and so did not probe this characterization. In the next session I made a point to ask them how they were measuring this "goodness", and they made clear that they were looking at how close the outputs of the function were to zero. This led to a discussion where we clarified that, at least for our problem, we were more interested in the accuracy of the inputs.

After the conclusion of each of the teaching experiments, I performed a retrospective analysis of the data as a whole. I watched all of the videos again, transcribing segments I had flagged during the ongoing analysis, looking for student strategies that anticipated the formal concepts of advanced calculus. While my analysis ultimately identified strategies pertaining to limits, completeness, and continuity, initially I was looking for any strategies pertaining to advanced calculus topics. After identifying those strategies, for each of one I sought to explain what elicited such student strategies. Finally, I followed these strategies through the data and using the design heuristics of RME I sought to 
explain how these strategies were leveraged to support the development of more formal ideas, or how they might be leveraged in future implementations of the LIT. See Appendix B for a sample spreadsheet of this analysis.

Now let us look at the results of these two teaching experiments, and how these results informed the refinement of the LIT.

\section{Results}

The data presented below, as well as the LIT presented later, are organized into three phases. This structure, originally inspired by Cauchy's proof of the IVT, has been modified from the preliminary LIT. This modification was based on two important insights gained from data analysis. First, the way the students engaged with and interpreted the tasks led me to re-frame the primary tasks in Phases 2 and 3. In the preliminary LIT, I had envisioned the proof of the IVT as encompassing Phases 2 and 3; Phase 2 focused on proving, using their algorithm, the existence of a root-candidate, while Phase 3 focused on proving, using continuity, that the root-candidate must be a root. Through the course of two teaching experiments it became clear that, in Phase 2, students were still assuming a root existed, and so did not envision their activity as proving existence. This led me to modify the nature of the tasks in the following way: 1) develop an algorithm that will approximate the root to any desired accuracy (i.e. find the root), and conjecture conditions under which your algorithm will be guaranteed to work; 2) assuming a root exists under your conditions, prove that 
your algorithm will find it, in general; 3) use your algorithm to prove that a root must exist under your conditions. In this new structure, the formal proof of the IVT occurs in Phase 3, and students more explicitly transition from using their algorithm for finding a root to using their algorithm to prove its existence.

The second motivation for modifying the phases, and more important for describing student development, was that through retrospective analysis I recognized that the students' approximation algorithm was an emergent model through the course of the task sequence. As they constructed an approximation algorithm to find the root of a polynomial, their reasoning and activity depended on both notions of convergence and completeness of the real numbers, even though the students themselves were not aware of these features. In this way their algorithm, with all of its theoretical underpinnings, served as a model-of their activity. By turning their attention and organizing efforts on the algorithm itself, students engaged in vertical mathematizing. This took the form of elaborating and making explicit the features of the problem, and of the algorithm itself, that allowed their approximation algorithm to work. By considering these justifications as conjectures and attempting to construct proofs of the same, students engaged in horizontal mathematizing at a new level of formality. The construction of these proofs supported the development of the model toward a model-for more formal reasoning. While not observed in the data due to time constraints, their approximation algorithm would be a key part of the proof of the IVT, and its use would evidence the transition to a model-for more formal reasoning on the part of 
the students. In this way the students' approximation algorithm could be seen as an emergent model through the course of the instructional sequence.

The design heuristic of emergent models offered further support for the utility of this modified three-phase structure in terms of the levels of activity. Student activity in Phase 1 begins in the situational realm, where they work to construct an algorithm to approximate the root of a specific function. In Phase 2 student activity occurs primarily in a referential capacity, as they investigate and generalize their algorithm. In Phase 3 student activity occurs at the general level, and the approximation algorithm transitions to a model-for more formal reasoning, as they use their new robust understanding of that algorithm to complete a proof of the IVT for continuous functions.

\section{Phase 1: Approximating the Root}

Student activity begins in the first phase at the situational level. There were two primary goals of this initial phase of the instructional sequence in the teaching experiments: 1) for students to develop an algorithm that would allow them to approximate the root of a continuous function to any desired degree of accuracy, and 2) to explicitly conjecture the sufficient conditions for such a root to exist. This student-generated algorithm would be one of the major objects of further investigation in the second phase, as students transitioned to the deductive phases of the instructional sequence. This transition to investigating 
the algorithm evidenced the transition to a referential level of activity on the part of the students.

With the opening task I asked students to determine whether or not a given polynomial had a root on a given interval. In both teaching experiments, the students essentially assumed the IVT (implicitly). This is evidenced by their argument that the polynomial must have a root in the interval because the signs of the outputs at each endpoint were different. Taking that as sufficient justification of the root's existence (for the moment), I next tasked the students with approximating this root to two decimal places (and then six decimal places, and then an arbitrary degree of accuracy). Using the heuristic of didactic phenomenology, this task was designed to provide students with a problem which the IVT would readily solve. In each of the teaching experiments the students were able to construct an algorithm that would allow them to approximate the root to any desired accuracy, by checking the sign of the function on increasingly fine intervals.

The remainder of the first phase of the instructional sequence was focused on the development and generalization of their approximation algorithm. The generalization of their algorithm began the deductive phase of the instructional sequence, and served as a bridge to the second phase, in which the algorithm itself became the object of study.

In each of the two teaching experiments, the pairs of students approximated the root of the given polynomial in distinct ways. While the 
algorithms they constructed appeared radically different on the surface, structurally they were really quite similar. After briefly describing the development of each algorithm and its final structure, the similarities and differences will be explored in more detail.

\section{Bisection Algorithm}

In the first teaching experiment, Brad and Matt argued that $p(x)=x^{4}-4 x^{3}$ $-7 x^{2}+22 x-10$ must have a root in the interval $[0,3]$ because the function went from positive to negative over the interval ${ }^{5}$. They employed a small handful of strategies to approximate this conjectured root, but their early efforts did not give them any handle on how close their approximations were to the root and were quickly abandoned. Eventually they settled on what I have called the Bisection Algorithm. Brad gave a brief description of this algorithm early in the third session:

$5 p(0)=10$, while $p(3)=-14$ 


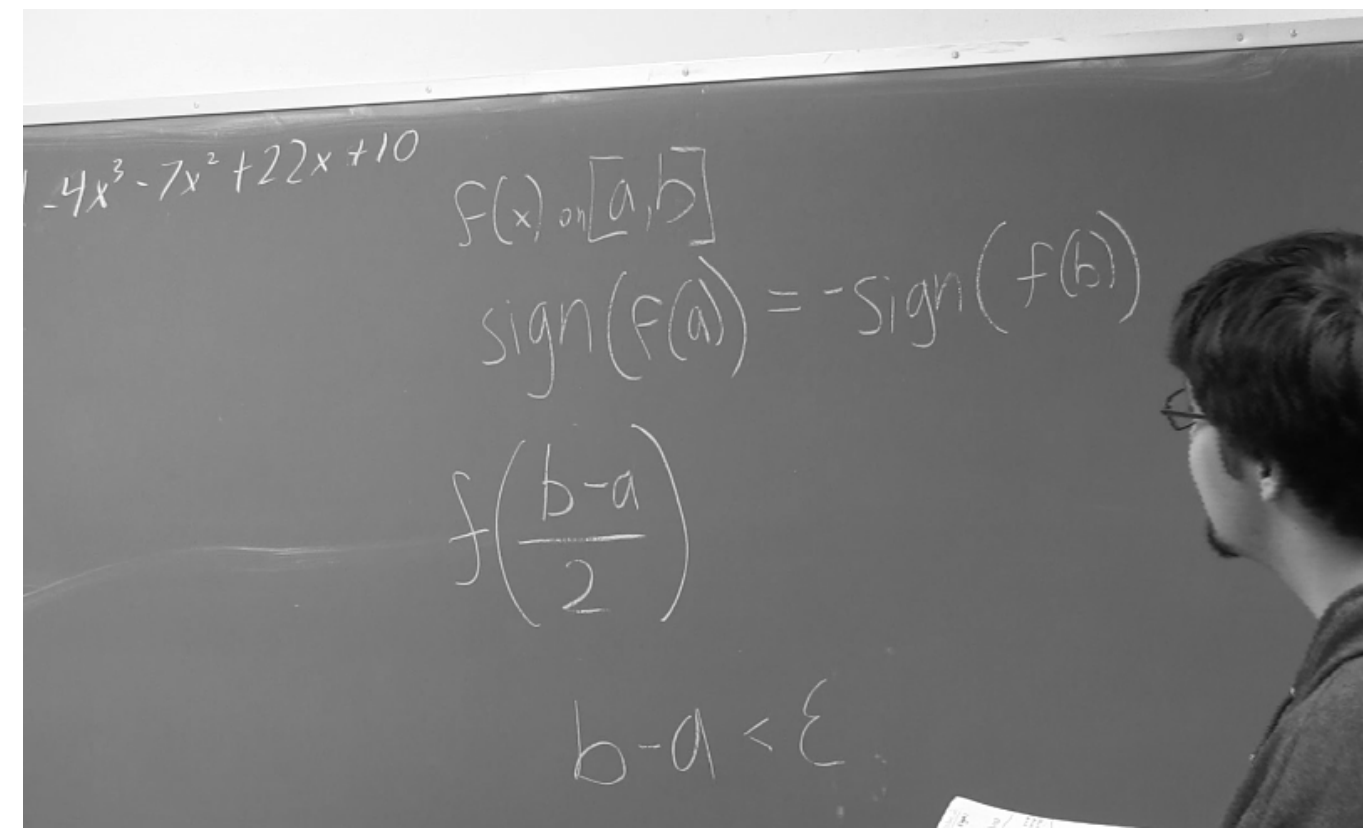

Figure 2: Brad and Matt's Bisection Algorithm

"So we tried to find, um, the average. And if this is greater than zero...wait. If this is equal to the sign of this one [pointing at 'sign $(f(a))^{\prime}$ '] then it becomes the new left endpoint. If it equals the sign of this one [pointing at 'sign $(f(b))$ '] it becomes the new right endpoint. And we continue doing it over and over and over and over again, until $b-a$ is so small that it's less than the error asked for."

One interesting feature of their algorithm at that point in the experiment was the use of the variables $a$ and $b$. In the original numerical example, Brad and Matt found the midpoint of each interval, using the length of the interval as an upper bound on the error in their approximation of the root. When they worked to generalize this algorithm, Brad chose to re-assign the values of $a$ and $b$ after each iteration of the algorithm (he explicitly referenced computer programming as the motivation for this). This did not become problematic for them until the next session, in which they were trying to provide algebraic arguments for some of 
their conjectures (e.g. the widths of the intervals go to zero, the endpoints approach each other, etc). At that point Brad suggested using $a_{n}$ and $b_{n}$ to refer to the sub-interval endpoints after $n$ iterations of their algorithm, and this was what they used moving forward.

After their first numerical approximations, they were less concerned with giving actual approximations as with bounding the error in possible approximations.

Brad: So, yeah. We're cutting- because we're starting here-

Matt: Mmhm.

Brad: -with our beginning endpoints, we take the average which is gonna cut it down in half. Either the new mean is going to be the left endpoint or the right endpoint.

Matt: Making that determination will change this interval every single time. Upon every single iteration. And this is the algorithm we were using to-

Brad: And so the difference, and thus the error, will be halved each time. Until we get to this point [gestures at work on the board], which is-

Matt: That's smaller than the asked for...the given interval that we were trying to find.

Brad: Correct. Or error.

This use of an error bound is a strategy that anticipates the formal, $\varepsilon-N$ definition of sequence convergence. For a sequence, $\left\{x_{n}\right\}$, converging to a real number $L$, the formal definition looks something like this:

For every real number $\varepsilon>0$, there exists an $N$ in the natural numbers so that if $n>N$, then $\left|x_{n}-L\right|<\varepsilon$.

One can think of the number $\varepsilon$ as a bound for the error, where in this case the error is the distance between the $n$th term of the sequence and its limit, $L$. 
The opening tasks of the instructional sequence were designed, using the heuristic of didactic phenomenology, to elicit this approximation strategy. By asking for specific, and increasingly precise, measures of accuracy, I expected students to find a way to bound the error in their approximation (since knowing the exact error was impossible without knowing the root). One simple solution to this problem was to recognize that the width of the interval would bound the error, as Brad and Matt did.

After reflecting on their algorithm in the specific case, and assigning variable names to relevant quantities, their final version of the general Bisection Algorithm could be described in this way:

1. Suppose $\operatorname{sign}(f(a))=-\operatorname{sign}(f(b))$ and that $f(x)$ is continuous on $[a, b]$. Then a root $r$ must exist $a<r<b$.

2. Check the midpoint of $a$ and $b$. Evaluate $f$ at this midpoint; it is either positive or it is negative ${ }^{6}$.

3. If it has the same sign as the left endpoint, it becomes the new left endpoint. Otherwise it becomes the new right endpoint. Because $f$ is continuous and the signs of the function at these new endpoints are different, a root must lie in this new smaller interval. Find the new midpoint and repeat.

4. Let $n$ denote the number of iterations of this algorithm, and denote the current interval endpoints by $a_{n}, b_{n}$.

5. Note that at each iteration the maximum possible error in choosing any point in the interval to approximate the root $r$ is bounded by the width of the current interval, or

$$
\left|a_{n}-b_{n}\right|=\frac{|a-b|}{2^{n}}
$$

In this way one can achieve any desired accuracy for the approximation to the root by performing a sufficiently large number of iterations of this algorithm.

6 Neither Brad nor Matt considered the case where the midpoint was the root. 
Let us turn to a similar but distinct algorithm that was developed by the pair of students in the second teaching experiment. After describing this algorithm's development and its final structure, I will briefly compare and contrast the Bisection and Decimal-Expansion algorithms. I will then discuss some of the formal mathematical concepts upon which these algorithms depended.

\section{Decimal-Expansion Algorithm}

Jay and Dylan argued that $p(x)=x^{5}-x-5$ must have a root in the interval $[0,2]$ because the outputs of the function had opposite signs at the endpoints ${ }^{7}$. Knowing that the root lay between 0 and 2, they proceeded to check the sign of $p(x)$ at $1,3 / 2,4 / 3$, and finally at 1.4 . Again, arguing from the sign change in the output of the function, they then stated that the root, $r$, must lay somewhere between $1.4<r<1.5$.

They then continued using what I call the Decimal-Expansion Algorithm. In order to approximate the root more systematically, they checked the sign of the output at successive digits at the current unknown decimal-place (e.g. 1.41, $1.42, \ldots)$. From the original interval they knew that $p(x)$ was negative to the left of the root, and positive to the right. Once the decimal digit at which the sign changed from negative to positive was found, go back to the previous digit. This represented the most accurate approximation so far. The next iteration of the algorithm involved moving to the next unknown decimal place and repeating this process. Three iterations of this algorithm are presented below (Figure 3).

$7 p(0)=-5$, while $p(2)=25$ 
First Iteration:

\begin{tabular}{|c|c|c|c|c|c|c|}
\hline $\boldsymbol{x}$ & 1.41 & 1.42 & 1.43 & 1.44 & 1.45 & 1.46 \\
\hline$+/-$ & - & - & - & - & - & + \\
\hline
\end{tabular}

Second Iteration:

\begin{tabular}{|c|c|c|}
\hline $\boldsymbol{x}$ & 1.451 & 1.452 \\
\hline$+/$ & - & + \\
\hline
\end{tabular}

Third Iteration:

\begin{tabular}{|c|c|c|c|c|c|c|c|c|c|c|}
\hline $\boldsymbol{x}$ & 1.4511 & 1.4512 & 1.4513 & 1.4514 & 1.4515 & 1.4516 & 1.4517 & 1.4518 & 1.4519 & 1.4520 \\
\hline$+/$ & - & - & - & - & - & - & - & - & - & + \\
\hline
\end{tabular}

Figure 3: The first few iterations of Dylan and Jay's Decimal-Expansion Algorithm

By the third iteration of the algorithm, the students were confident that the root must lie between $1.4519<r<1.4520$. Dylan wrote out a brief explanation of their algorithm on the board in the middle of the first session.

"The root must be between 1 and 2 because $p$ crosses from - to + , therefore it crosses the $x$-axis.

By the same logic, root must be between $1.3(-) \& 1.5(+)$, so we evaluated increasing values from 1.3 and since there are no holes or asymptotoes [sic] the limit from the left is the same as the one from the right. Each time a value went over 0 , we went to the previous value and added another decimal place."

In this way the students were able to approximate the desired root, one decimal place at a time, and were confident they could achieve any degree of accuracy desired. 
While the implementations were rather different, these two algorithms were actually quite similar. The primary idea behind each of them was that by considering smaller and smaller intervals, and evaluating the sign of the function at the endpoints of those intervals, one could essentially "zoom in" on the root. The Bisection Algorithm did this by cutting the interval into two equal pieces at each iteration, thereby halving the maximum possible error. The DecimalExpansion Algorithm broke the interval into ten equal pieces at each iteration, dividing the maximum possible error by $\operatorname{ten}^{8}$. In either case the width of the subinterval could serve as a bound for the error in a given approximation. Furthermore, the convergence of the sequences generated by each of these algorithms depended on the completeness of the real numbers.

The major differences between these algorithms lay in the students' implementation and interpretations of these algorithms. While Brad and Matt explicitly considered the sub-intervals, and their widths as error-bounds, Dylan and Jay did not. In their implementation of the Decimal-Expansion algorithm Dylan and Jay focused entirely on the decimal values of their expansion; neither intervals nor error-bounds were ever explicitly mentioned. Brad and Matt argued that their algorithm converged to the root because the widths of the sub-intervals went to zero. Dylan and Jay argued that their sequence of approximations converged to the root because these approximations were monotonically increasing and bounded above by the root. These differences led to different

8 It should be noted that neither Dylan nor Jay ever referenced subdividing intervals, or dividing the maximum possible error by a factor of 10 . 
investigations in Phase 2, but ultimately supported each pair of students in constructing a formal definition of sequence convergence.

\section{Summary of Phase 1}

Here in Phase 1 of each of the teaching experiments we saw students develop algorithms for approximating the root of a continuous function to any desired accuracy. This situational activity with respect to the algorithm laid the foundation for the referential activity of Phase 2. Already strategies that anticipate formal concepts in advanced calculus had begun to emerge. We saw Brad and Matt utilize error-bounds to justify why their algorithm could approximate the root of a continuous function to any desired accuracy. They further stated that the error-bound going to zero would result in a single real number. With Dylan and Jay, on the other hand, we saw them argue that their sequence of approximations converged to the root because these approximations were monotonically increasing and bounded above by the root.

These strategies are essentially informal characterizations of the completeness of the real numbers. In the case of Brad and Matt, their strategy was essentially the Nested Interval Property, while with Dylan and Jay their strategy anticipated the Monotone Convergence Theorem (see Appendix A). In the case of Dylan and Jay, these justifications will be analyzed and codified into an explicit conjecture (the MCT). 
The investigation of these conjectures aligns well with the heuristic of didactic phenomenology. Two concepts that lie at the heart of the IVT (and advanced calculus in general) are convergence and completeness. The former provides a solid footing for the mathematical constructs defined in terms of limiting processes (derivative, integral, Taylor series, etc.), while the latter assures us that using the real numbers for these limiting processes is a reasonable thing to do. In particular, the students' approximation algorithm generates a sequence, one whose convergence depends upon completeness of the real numbers. For this reason, in order to prove their own conjectures about the nature of this convergence, students need to develop both a formal definition of sequence convergence and a formal characterization of the completeness of the real numbers. In this way these critical concepts are developed to solve a concrete problem for the students.

Finally, the students' approximation algorithms are themselves strategies that anticipate the proof techniques of the IVT, and as such can also be thought of as emergent models. In Phase 1 students engaged in situational activity, by horizontally mathematizing the problem of approximating the root of a polynomial. Let us now turn to the second phase of each of the teaching experiments, in which the students engaged in vertical mathematizing as they began to investigate their algorithms, transitioning to activity at the referential level. 


\section{Phase 2: Justifying the Algorithm}

The primary task of this second phase was the following: Given that there is a root, how do you know that your algorithm will find it? To initiate this second phase students engaged in vertical mathematizing, as the focus of study shifted from approximating the root of a specific polynomial to the student-generated algorithm itself. In this way their activity transitioned to a referential level with respect to their algorithm. Throughout this phase student activity took on more formal, deductive characteristics, as they generated and modified both definitions and conjectures related to the IVT.

It should be noted that the above task implicitly involves two steps: 1) using the algorithm to find a root-candidate, and 2) proving that the rootcandidate really is a root. Below I will describe how the students engaged with that first part, with the second part being the focus of the third and final phase.

Students began the transition to the second phase of the instructional sequence by engaging with the task: Given that your algorithm will never tell you the exact root, how do you know there is such a number? When justifying the convergence to a root of the polynomial of one or more of the sequences generated by their algorithm, students made arguments that were essentially informal characterizations of the completeness of the real numbers. Of course, they never explicitly acknowledged that the real numbers needed to be complete. This is not surprising given that they had likely never been exposed to this concept; Cauchy himself did not make reference to the completeness of the real 
numbers in many of his early formal proofs (Grabiner, 1981; Lützen, 2003). Let us consider the justifications made by each pair of students in turn.

Both of the student-generated algorithms offered a number of potential sequences of approximations to consider. In the case of the Bisection Algorithm, Brad and Matt focused on an interval around the root, whose length is halved with each iteration. The students also described their algorithm as a process by which both endpoints of the interval approached the root, getting closer with each iteration of the algorithm.

Matt: Because we were establishing over here that, $b_{n}$ and $a_{n}$ will get smaller [sic] upon more and more iterations. They'll become closer in value to each other. To the point where, after enough iterationswell, infinity iterations, $b_{n}=a_{n}$.

Brad: Okay. I definitely agree that this $\left[\left|b_{n}-a_{n}\right|\right]$ is going to zero as $n$ goes to infinity. It will never reach it but it will get very very very close. 


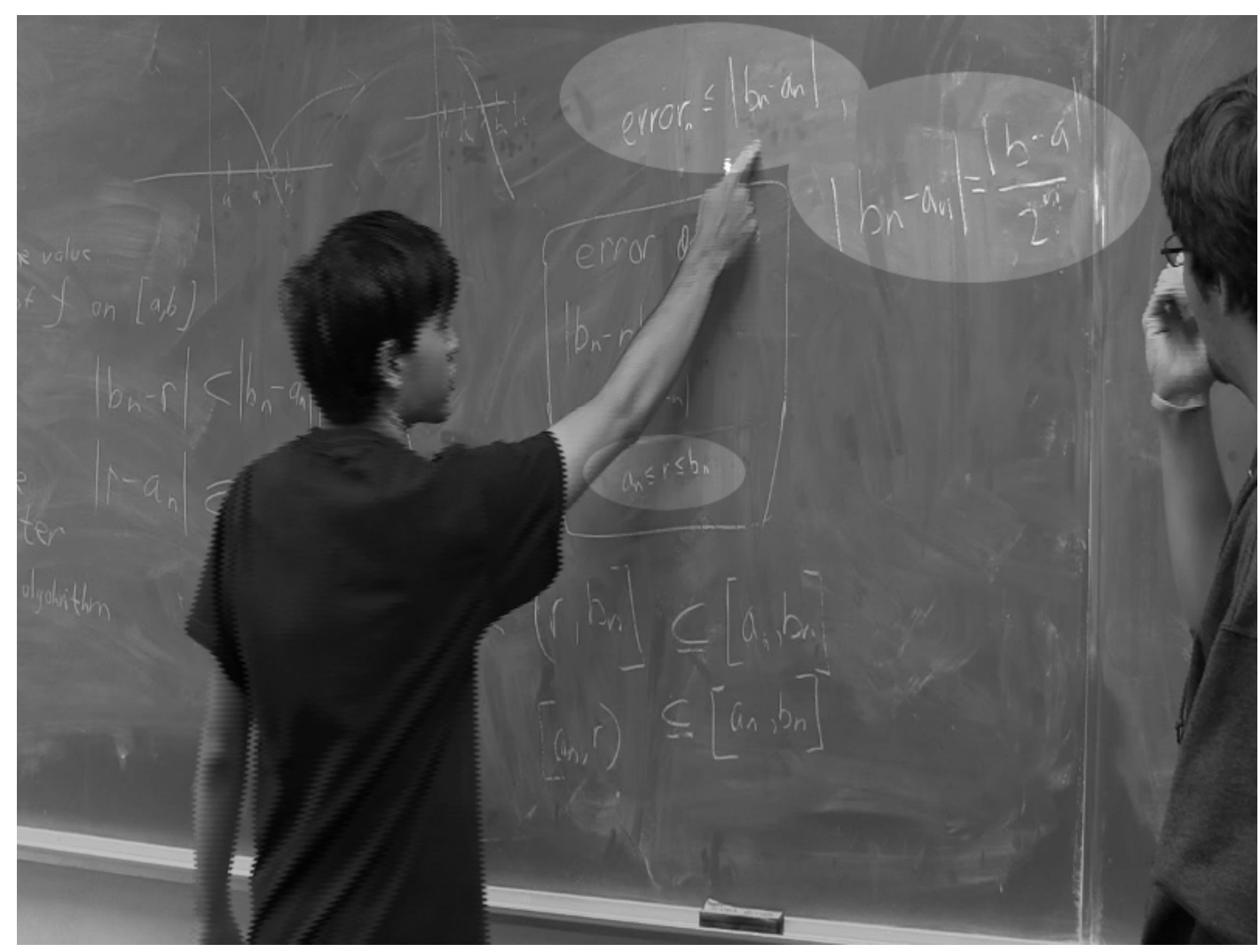

Figure 4: In the highlighted expression near Matt's elbow, the root $r$ is always trapped inside this shrinking interval, between the endpoints $a_{n}$ and $b_{n}$, the distance between which also bounds the error in their approximation at the nth step.

The students' assertion that the error-bound for their approximation of the root "went to zero" served as their primary justification for the convergence of their algorithm. Though Brad and Matt have labeled the endpoints of the sub-intervals as $b_{n}$ and $a_{n}$, notation which suggested sequences, at that point in the experiment they had not explicitly referred to the endpoints as sequences themselves. Rather they spoke of the shrinking interval as the thing that was approaching the root. 
When asked to justify their assertion that their algorithm converged to the desired root, Brad and Matt argued that the lengths of the sub-intervals went to zero in the limit. The idea that this process would result in a single real number is essentially the Nested Interval Property (see Appendix A) of the real numbers, which is itself one of many ways to characterize that the real numbers are complete. A retrospective analysis suggested that having the students work to analyze and prove such a conjecture could be a fruitful way to motivate a formal definition of sequence convergence, as well as support them in developing their understanding of the completeness of the real numbers. For these reasons this activity became the focus of Phase 2 in the second teaching experiment. I will now describe some of the key steps in how Dylan and Jay engaged in this process of proof construction and analysis.

The Decimal-Expansion Algorithm provided similar but distinct opportunities for Dylan and Jay to investigate the concepts of "approaching" and "convergence". By considering successively more accurate decimal approximations to the root (e.g. 1.4, 1.45, 1.451, 1.4519,...), Dylan and Jay chose to construct a sequence of approximations that monotonically increased toward the root. In this case the sequence generated by the algorithm was a monotonically increasing sequence bounded above by the root (or by any of the $x$-values which surpassed the root). That such a sequence must converge seemed intuitively obvious to them. This focus, by the students, on the monotonicity of their approximations served as the launching point for their 
investigations in Phase 2.

In what follows I will describe the key steps in the conjecture and construction of Dylan and Jay's MCT. For a fuller description of this process, please see Completeness and Sequence Convergence: Interdependent Development in the Context of Proving the Intermediate Value Theorem (Strand, in preparation).

\section{Step 1: Justifying the algorithm}

Dylan and Jay had remarked that, since their algorithm gave them one decimal at a time, if the root were irrational this algorithm could never give it exactly. This prompted the following exchange.

I: So how do you know that there is such a number?

D: As long as we can recursively show that every time we step our function forward it gets a little bit closer to zero. This is how you do the limit in general: every time you step it forward, every time you know you move forward a little bit, you get closer to the number you think the limit is.

Notice that Dylan's statement, though fraught with what some would call "misconceptions", was true in reference to the specific polynomial they had been considering. Regardless of whether Dylan actually believed his statement characterized limits in general, I saw the seeds of the Monotone Convergence Theorem (MCT) in his reasoning. This led me to hypothesize that pursuing this reasoning could give insight into the students' thinking about completeness, and perhaps in supporting the students in formalizing this concept. In an effort to 
isolate the key features of their reasoning, I asked Dylan and Jay if a similar statement could be made about the inputs. Dylan wrote

$$
x_{i}<x_{i+1}<\bar{x},
$$

where $\bar{x}$ stood for the conjectured root.

I next wanted to engage Dylan and Jay in vertical mathematizing. Recall that vertical mathematization involves reflecting on and organizing one's own mathematical activity. I codified this reasoning as a general conjecture and asked the students to consider it. In retrospect, it would have been more valuable to the students to codify this conjecture for themselves. However, my intention was that considering this conjecture that I provided would cause Dylan and Jay to analyze and organize their own reasoning with respect to their sequence of approximations. Let us now consider their activity in this process.

\section{Step 2: Conjecturing conditions to converge to a bound}

I wrote the following modified compound inequality on the board.

$$
x_{i}<x_{i+1}<b
$$

The first conjecture I had them investigate was whether or not the above compound inequality meant that the sequence $\left\{x_{i}\right\}$ necessarily converged to the bound, $b$. After a few moments of individual thinking time, Jay concluded that such a sequence need not converge to just any bound. He wrote

$$
x_{i}<x_{i+1}<1000
$$

and then explained (italics indicate student emphasis): 
Jay: My thing was, uh, you could have $x_{i}$ less than $x_{i+1}$ less than, let's say, a thousand. But this sequence, it doesn't necessarily have to converge to a thousand. This would be satisfied if it converged to two.

Dylan: Yeah, that was my first problem: it doesn't necessarily converge to $b$. What I think is interesting is that this does mean that it necessarily converges. That it never passes some value.

Here we see the Monotone Convergence Theorem, even more explicitly in Dylan's reasoning. He then went on to explain his thinking further, and to conjecture some conditions for when the sequence would converge to $b$.

"Because if you can pick a value, some $a$, between $x_{i+1}$ and $b \ldots$ and $\ldots x_{i+1}$ passes every value of $a$...like every possible value of $a$...and passes $b$...wait, if this is true, so it doesn't pass $b$. So worst case scenario it converges to $b . "$

It appears that what Dylan described was essentially a characterization of $b$ as the least-upper bound of the sequence. He seems to suggest that if we could choose $a$ to be any arbitrary value less than $b$, and then we knew that a value of the sequence $\left\{x_{i}\right\}$ passed that value of $a$, then the sequence would have to converge to $b$. So there was no value of $a$ less than $b$ that was also an upper bound for the sequence. When I told Dylan and Jay that this was one way to characterize that $b$ was the least-upper bound of the sequence, Dylan verbally interpreted that statement in the following way: "You can pick any number bigger than $b$, and this inequality would also be true. But you can't pick a number smaller than $b . "$

This condition, that the sequence passed every value of a less than $b$, but 
never passed $b$, struck me as a powerful insight. For that reason I codified it and added it as a hypothesis to their conjecture. This condition would later become a transformational record, when it directly and explicitly influenced their eventual formal definition of a sequence decreasing to zero.

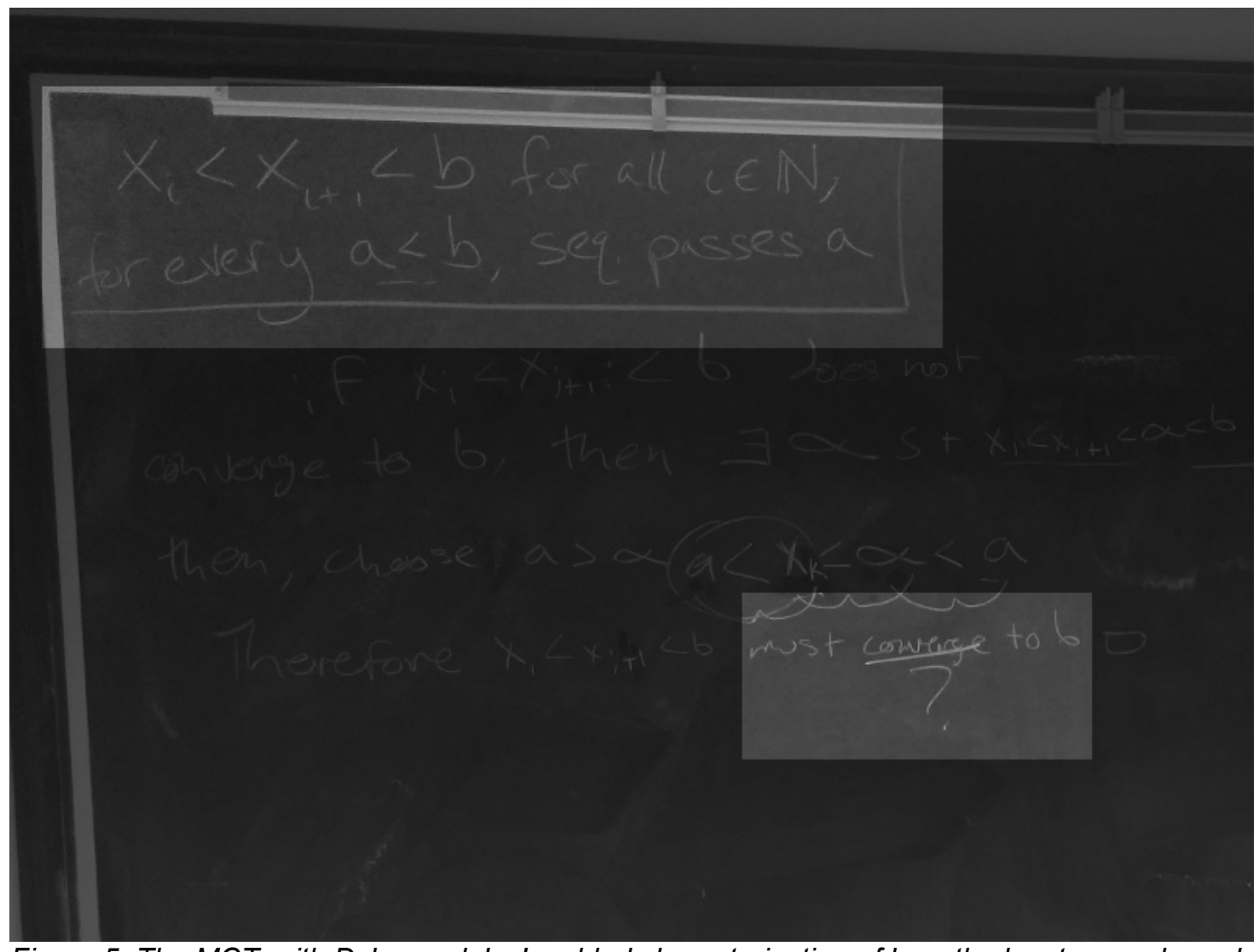

Figure 5: The MCT, with Dylan and Jay's added characterization of $b$ as the least-upper bound.

Dylan and Jay then considered whether this extra condition, that the sequence passed every value of a that was less than $b$, would be enough to guarantee convergence to $b$. While they both agreed that it should, they realized 
that they needed a more formal definition of "converges".

\section{Step 3: Developing a formal definition of sequence convergence}

Of their own volition, Dylan and Jay chose to consider a damped sine curve in order to try and capture "convergence" (Figure 6). In their very first attempt, Dylan and Jay opted to characterize convergence in terms of a sequence of "errors". They called these errors term " $\varepsilon_{i}$ ", and defined it as the difference between the greatest and least outputs of the sequence from some point, $i$, on. In Figure 6, Jay had labeled the first three of these "errors", corresponding to $x_{i}, x_{i+1}$, and $x_{i+2}$ (though the peak for $\varepsilon_{2}$ appears to be mislabeled; it should follow $x_{i+1}$ ).

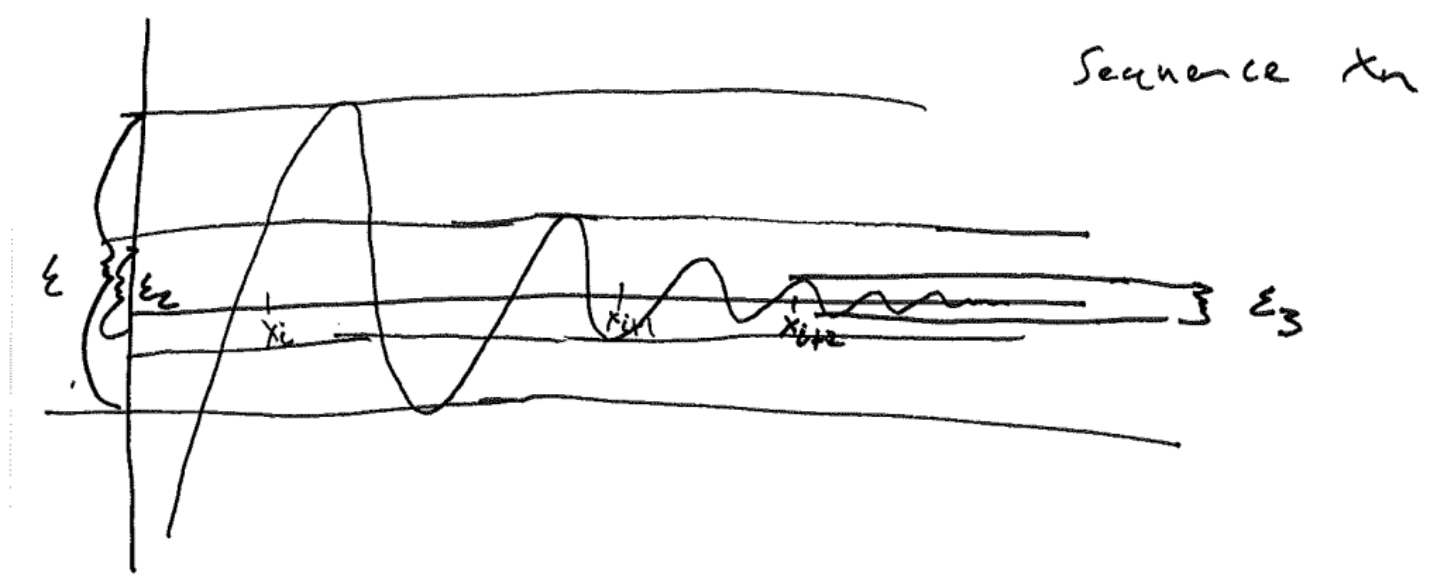

Figure 6: Jay's sketch of the damped sine curve, which he and Dylan used as their prototypical example of convergence.

Dylan verbally described this "error", in terms of $\varepsilon$, in the following way: 
"So this $\varepsilon$ is...let's just define $\varepsilon$ of $x_{i}$ is the biggest value the sequence- the difference between the biggest and smallest value the sequence takes from $x_{i}$ forward. Does that sound fair?"

Notice that in both Dylan's statement and Jay's sketch they have indexed by the outputs of their sequence $\left\{x_{i}\right\}$, rather than by $i$ itself. This was simply an oversight on their part, and they quickly corrected it when I pointed it out. They formally codified this definition (Figure 7), and after some discussion agreed that the sequence $\left\{x_{n}\right\}$ should converge provided that the sequence of $\varepsilon$ 's went to zero. Dylan and Jay recognized that they had just defined "convergence" by using convergence of a different type, which motivated us to formally define what was meant by "goes to zero".

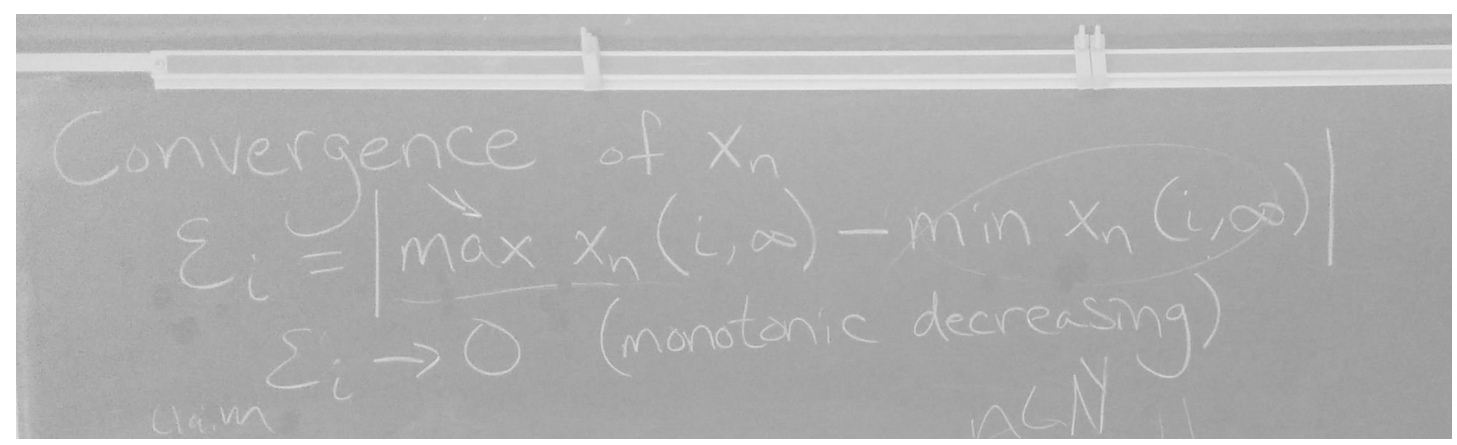

Figure 7: Dylan and Jay's formal definition of sequence convergence.

This definition of convergence in terms of a sequence of "errors" appeared quite cumbersome, but aside from their use of "max" and "min" (which was problematic because not all sequences have maximum or minimum elements) it 
was logically sound. And in fact, when Dylan and Jay were tasked with defining how they were using "max", it became evident that they were thinking of a leastupper bound (and similarly "greatest-lower bound" for $\mathrm{min}$ ). This prompted me to introduce the terms "supremum" and "infimum", and this was how they used their definition in the proof of the MCT.

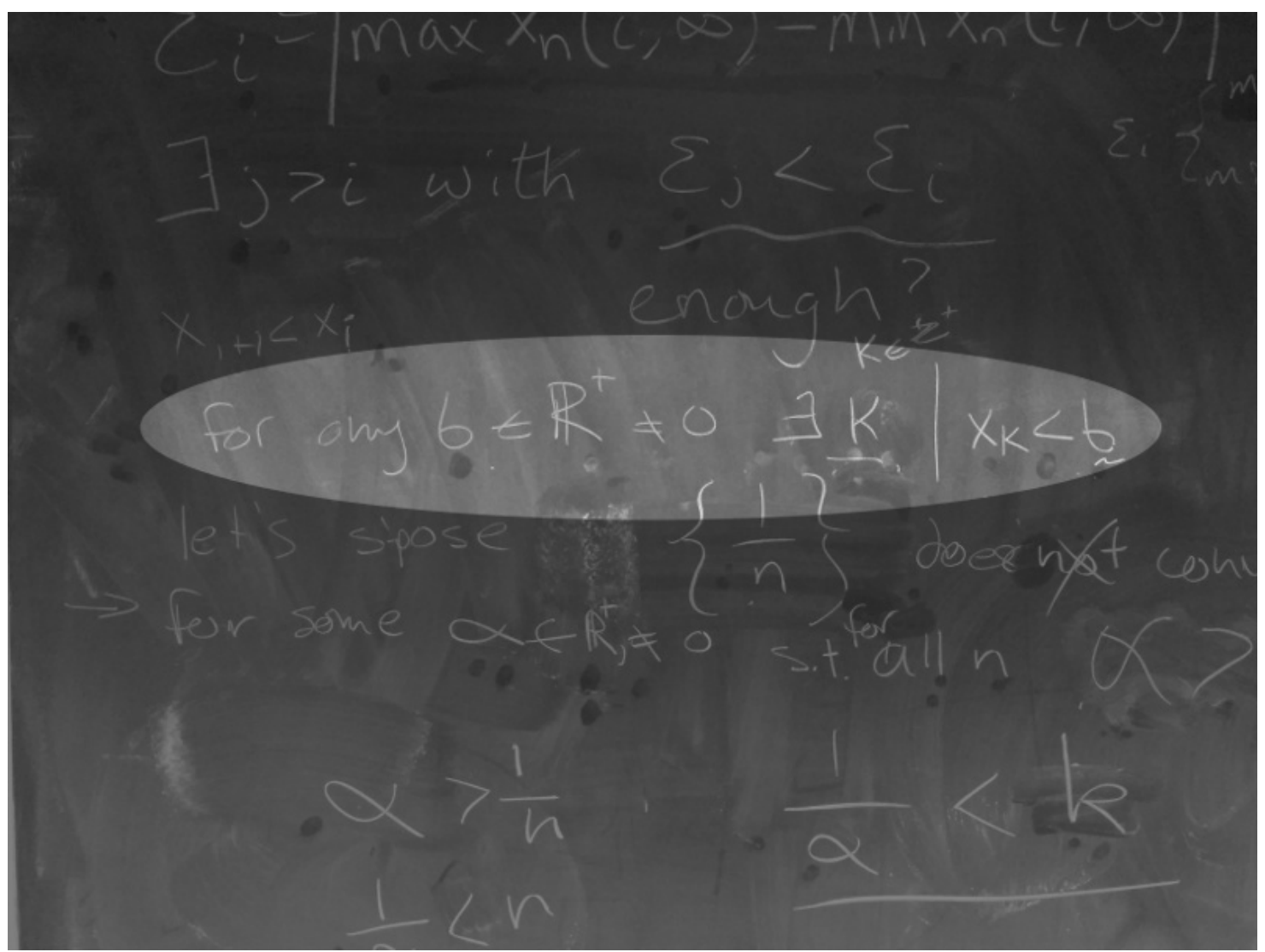

Figure 8: Dylan and Jay's criteria for a sequence to "decrease to zero".

In defining "decreases to zero", Dylan explicitly drew on their earlier work with the modified MCT. Recall that I had added the extra hypothesis that the 
sequence "passed every a less than $b$ ". Dylan adapted this condition to a sequence monotonically decreasing to zero: in order to converge to zero, such a sequence would have to pass every positive real number (represented as $b$ in Figure 8). He then explained this definition:

"So the boundary we know we want is zero. So we're going to talk about all the numbers that aren't zero, above zero. So these numbers [gestures at his definition, "positive reals not equal to zero"]...So we know this is always getting smaller. Down to some...you know, whatever. It goes off to somewhere. But do you know it goes to zero? And you do as long as you can pick any of these numbers [positive real numbers] and just keep going through until you find some $k$ [sic] that's smaller than it."

The earlier hypothesis involving "passes every a less than $b$ " had become a transformational record: it was an inscription of student thinking that the students subsequently used as a tool to achieve a mathematical goal, namely that of formally defining a sequence decreasing to zero.

With a formal definition of convergence (in two parts), we returned to the original statement of their conjectured MCT and attacked the proof.

\section{Step 4: Completing the proof of their MCT}

Recall that the statement of their MCT read simply:

If there exists a ' $b$ ' in the reals s.t. $x_{i}<x_{i+1}<b$ for all ' $i$ ' in the Naturals, then $\left\{x_{n}\right\}$ converges.

To prove this using their definition in terms of a sequence of "errors", they needed to identify the supremum and infimum of the sequence from some point on, and 
then show that the difference between those values decreased to zero. They readily recognized that because the sequence was monotonically increasing, the infimum from some point on would always be the current sequence value; that is, the infimum of the sequence from $i$ on would be $x_{i}$.

The critical piece of the proof came when Dylan and Jay tried to identify the supremum of the sequence $\left\{x_{n}\right\}$. Dylan wanted to call this value $L$, but Jay was not comfortable simply assuming that such an $L$ would exist.

"My problem with using the sup, is that you assume that this- it's like you're assuming it converges...So if there's nothing bigger than it, and... I can always get as close as I want to it. So you're basically arguing that it converges."

In some sense Jay was correct. Assuming the existence of the supremum was the key step in completing the proof, and he had basically outlined the remainder of the argument. Dylan, though, felt differently: "But I think we can make an argument that the sup exists, from just knowing that there's some maximum bound." His argument relied on his intuitive understanding of the real numbers, and in particular their representation via the real number line. He argued that, if the given upper bound $b$ was not the least upper bound, then there had to be a smaller upper bound, c. (Italics in the transcript represent the student's emphasis.) 


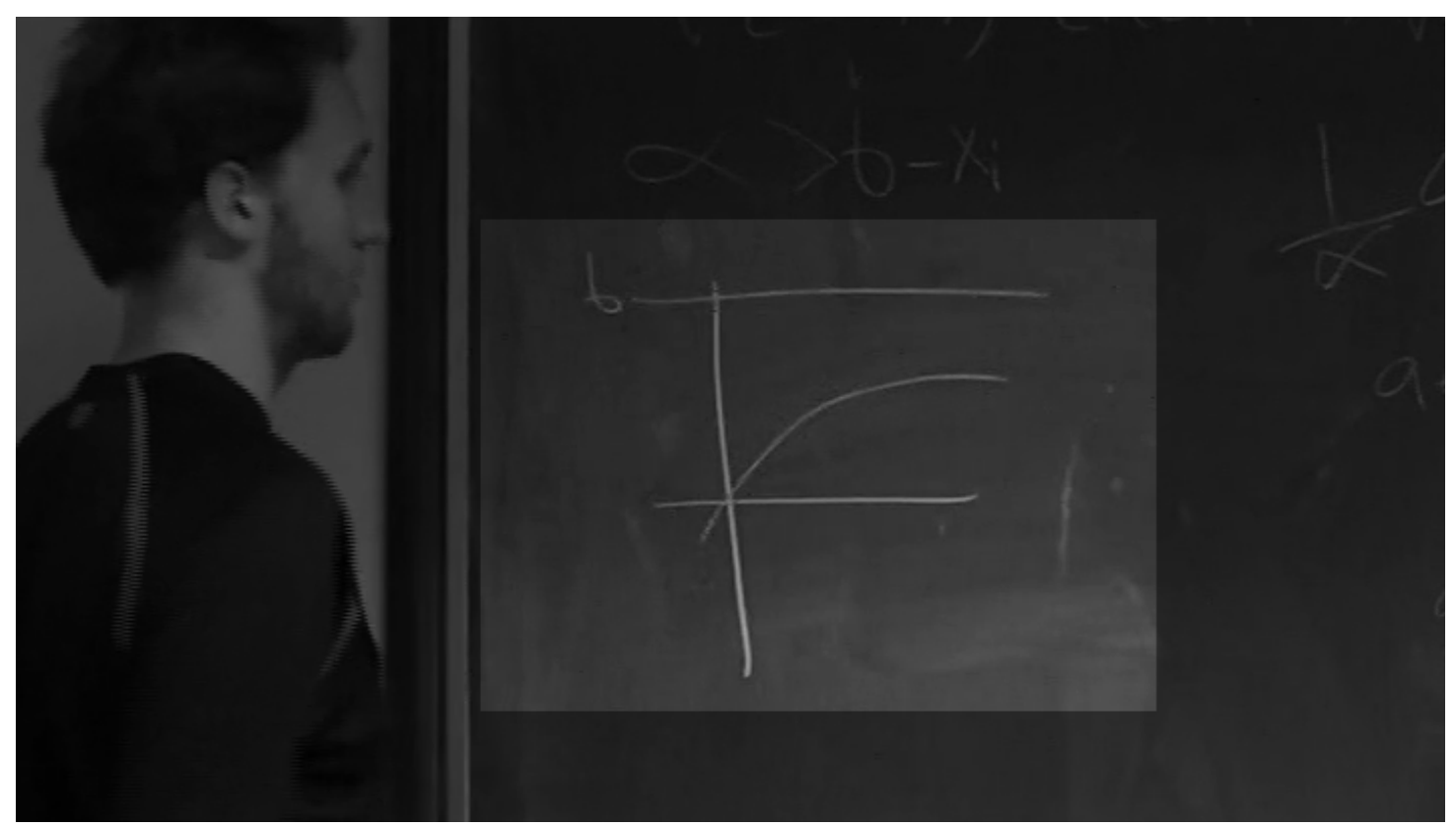

Figure 9: Dylan's sketch arguing why the least-upper bound of the sequence must exist.

Jay: But we don't know that there's another-

Dylan: There has to be. That's what I'm saying. By the properties of the real numbers here. If there- if this $\left[x_{i+1}\right]$ never gets- like, if I pick a number that's smaller than $b$, that this value $\left[x_{i+1}\right]$ is never bigger than, then- I guess that's how a number line works, right? Let's say it does this kind of increasing behavior [Figure 9], but this is our $b$, so there is some value $c$. That's less than $b$. If this statement [upper bound inequality] is also true, if we can replace some $c$ for this $b$ ? And this inequality remains true? We keep doing that.

Jay: Yeah. I can deal with that.

At that point Jay was convinced (or at least capitulated), and he and Dylan agreed to call the supremum $L$. In this way the students identified a characterization of completeness that they were willing to take as an assumption without proof. As my primary goal was simply to engage students in a single instance of justifying a characterization of completeness (i.e., using the existence 
of least-upper bounds to justify the MCT), I did not push them for further justification. Additionally, many authors of real analysis textbooks use the leastupper bound property as their axiom of completeness for the real numbers, or as its primary characterization in the case of construction (Bartle \& Sherbert, 2000; Krantz, 2013; Ross, 1980; Rudin, 1964; Wade, 2004), and so it seemed to me a reasonable foundation for the students to use.

All that remained to finish the proof was for Dylan and Jay to show that, given an arbitrary positive real number $\alpha$,

$$
\alpha>\left|L-x_{i}\right|
$$

They rearranged that statement to the following inequality,

$$
x_{i}>L-\alpha
$$

Which they interpreted as: are there elements of the sequence within $\alpha$ of $L$ ? Proceeding by contradiction, they supposed that there were not. This gave them the next inequality:

$$
x_{i} \leq L-\alpha,
$$

which had to be true for all elements of the sequence $\left\{x_{n}\right\}$. Dylan and Jay then recognized that this was not possible: the above inequality would imply that $L$ - $\alpha$ was an upper bound for the sequence, and one that was smaller than $L$. But $L$ was chosen as the least-upper bound, so this must be a contradiction. This completed the proof of their MCT.

With the proof completed, and with their newly constructed understandings of sequence convergence and completeness, I asked Dylan and Jay to once 
again return to their algorithm and the sequence of approximations it generated. I then tasked them with formally proving that their algorithm would find a root. Interestingly, rather than applying the Monotone Convergence Theorem directly, Dylan and Jay used the techniques from their proof. They first verified that their sequence of approximations was monotonically increasing, and that it was bounded above. By their previous argument, this allowed them to conclude that there was a least-upper bound for their approximations. In a similar proof by contradiction, they formally showed that their sequence of approximations must converge to that least-upper bound. This marked the end of the second phase of the instructional sequence, and the question of why such a number must be a root motivated the transition to the third and final phase.

Having formally proven that their algorithm would find a number, Dylan and Jay were ready to begin the third and final phase of the instructional sequence: proving that their conditions guaranteed the existence of a root.

Though Dylan and Jay did not complete this proof in the teaching experiment, we will consider their initial attempts to formalize their notion of continuity.

\section{Summary of Phase 2}

Activity in the second phase of the instructional sequence comprised the bulk of both teaching experiments. Here in Phase 2 student activity transitioned to the referential level, as they referred back to their activity in the first phase to investigate how and why their algorithm worked. 
With Brad and Matt, we saw how the justifications that their algorithm would find a root were essentially the Nested Interval Property. Unfortunately at the time I did not recognize this characterization in their thinking; only in retrospective analysis did this characterization become evident to me. This caused me to modify the LIT (and the instructional sequence) for the second teaching experiment. Specifically, I had Dylan and Jay more explicitly address (and ultimately prove) why their algorithm would find a number.

In the case of Dylan and Jay, this involved proving the Monotone Convergence Theorem (MCT). We saw how they justified their belief that their sequence of approximations converged by appealing to the fact that it was increasing and bounded above. In order to dig into their informal conceptions of completeness, as well as to provide the need for them to develop a formal definition of sequence convergence, I codified this justification as an explicit conjecture (their MCT) and had the students try to prove it. The proof of the MCT requires two main steps: to use a formal definition of sequence convergence, and to appeal to another characterization of the completeness of the real numbers. In terms of didactic phenomenology, this provided the students with a need not only to develop a formal definition of what it meant for a sequence to converge, but also to formulate another characterization of completeness. Through this process Dylan and Jay uncovered the need for the existence of least-upper bounds (a characterization of completeness they accepted without proof). Using this property, along with their definition of sequence convergence, they were able 
successfully to complete their proof of the MCT.

Now let us turn to the third and final phase of the instructional sequence. Here we will examine the informal conceptions of continuity expressed by both pairs of students. We will then consider how Dylan and Jay made the first steps in formalizing the idea that a continuous function has no "jumps" or "breaks", and this development's implications for the LIT.

\section{Phase 3: Proving Existence of the Root}

Originally (as we will see in the data) I had intended the third phase of the instructional sequence to focus solely on continuity, and its role in proving the IVT. With the tasks in the second phase I expected students to develop a solid understanding of why and how their algorithm would find a number that would serve as a candidate for a root. However, in analyzing the data it became evident that the students acted as if a root must exist, and that the job of their algorithm was simply to find it. When I describe the local instructional theory in the succeeding section, I will detail my current thinking about the structure of this third phase. For now, let us examine the informal characterizations of continuity given by each pair of students, as they wrestled with the role of continuity in their proof of the IVT.

In both teaching experiments, initially the existence of a root seemed to be an obvious consequence of the continuity of the given function; this was not at all 
surprising, given the students' experiences with functions, as well as the historical development of the IVT. Let us look at the students' justifications for the existence of a root when they were first putting together, and then generalizing, their approximation algorithms.

In the first teaching experiment, working on the opening task, Brad and Matt quickly recognized that the existence of a root relied on the continuity of the function in question.

Brad: So if we have it going from positive to negative, is has to hit zero at some point.

Matt: Yeah.

Brad: Is it the Mean Value Theorem, I think? Whatever it is. But from positive to negative it has to hit zero in between. If it's continuous at least, which it is.

Matt: Yes. All polynomials are continuous.

A few minutes later, the two students provided two different explanations for what it meant for a function to be continuous.

I: $\quad$ So, we may not want to dig into this just yet, but what does "continuous" mean?

B: Do you remember the definition at all?

M: The idea of continuous...for a function or a graph is that there are no spontaneous jumps from one $x$, from one- actually, one $x$-value or input value to another.

B: Yeah, so there can't be a hole or an asymptote that splits it all up.

M: A vertical asymptote, yeah.

B: $\quad$ Right. Or, I think...I remember hearing some sort of technical definition. I think it's at every point $x$, there has to be an $f(x)$, it has to have a limit from both sides,-

M: $\quad$-Correct.

B: $\quad$-and the limit has to equal the point.

M: Yes, that's right.

B: So, at any point there's something that exists, 'cause there's no holes. How we justify that I have no idea. And then the limit, you go 
from the left and right it will always hit where that point is.

Brad and Matt gave two standard characterizations of what it meant for a function to be continuous, at least as encountered in an undergraduate calculus course. I will explore each of these characterizations in greater depth when I describe the LIT in the next section.

In the second teaching experiment, Dylan and Jay spontaneously gave very similar justifications for the existence of a root, and similar characterizations of continuity.

I: What does continuous mean?

Dylan: There aren't any breaks and any jumps in the function, any corners where it would change suddenly. And there aren't any holes- there aren't any places basically where you can't evaluate the function.

Jay: If I remember from calculus correctly, it also has to do with- if you have- you make sure that at every point, every point that the lefthand limit and the right-hand are always converging to the same spot for every single spot. At least on your interval.

Notice that Dylan had added the extra condition that the function not have "any corners where it would change suddenly". A short while later, he and Jay would eventually agree that this condition actually described differentiability, and was not necessary for their algorithm to work. Interestingly, while both Dylan and Jay recalled the limit characterization, neither of them appealed to it when attempting to formalize their definition of continuity.

Unfortunately, with Dylan and Jay we did not have a great deal of time for them to explore and refine their understanding of continuity. However, they made 
some insightful and illuminating statements that suggested some promising routes for the emerging LIT. It was clear that, to Jay, the IVT followed trivially from the continuity of a function:

"If $f$ is continuous - that means there's no breaks or nothing, just...from $a$ to $b$ it's a line, it's a curve - and let's say, we'll do the first case here, $f$ is less than zero, then $f$ is greater than zero, and it's continuous, you have- you have to cross zero."

Though when pressed, Jay agreed that perhaps a more precise definition of continuity would help us to understand why this would be so. This quote highlights one of the fundamental difficulties in an advanced calculus course: putting together formal proofs of what seem to be obvious results.

After this discussion Dylan and Jay attempted to write a formal definition of continuity on the board (Figure 10). They seemed to be trying to capture Dylan's statement that continuity meant, "you could walk from one point to the next": 


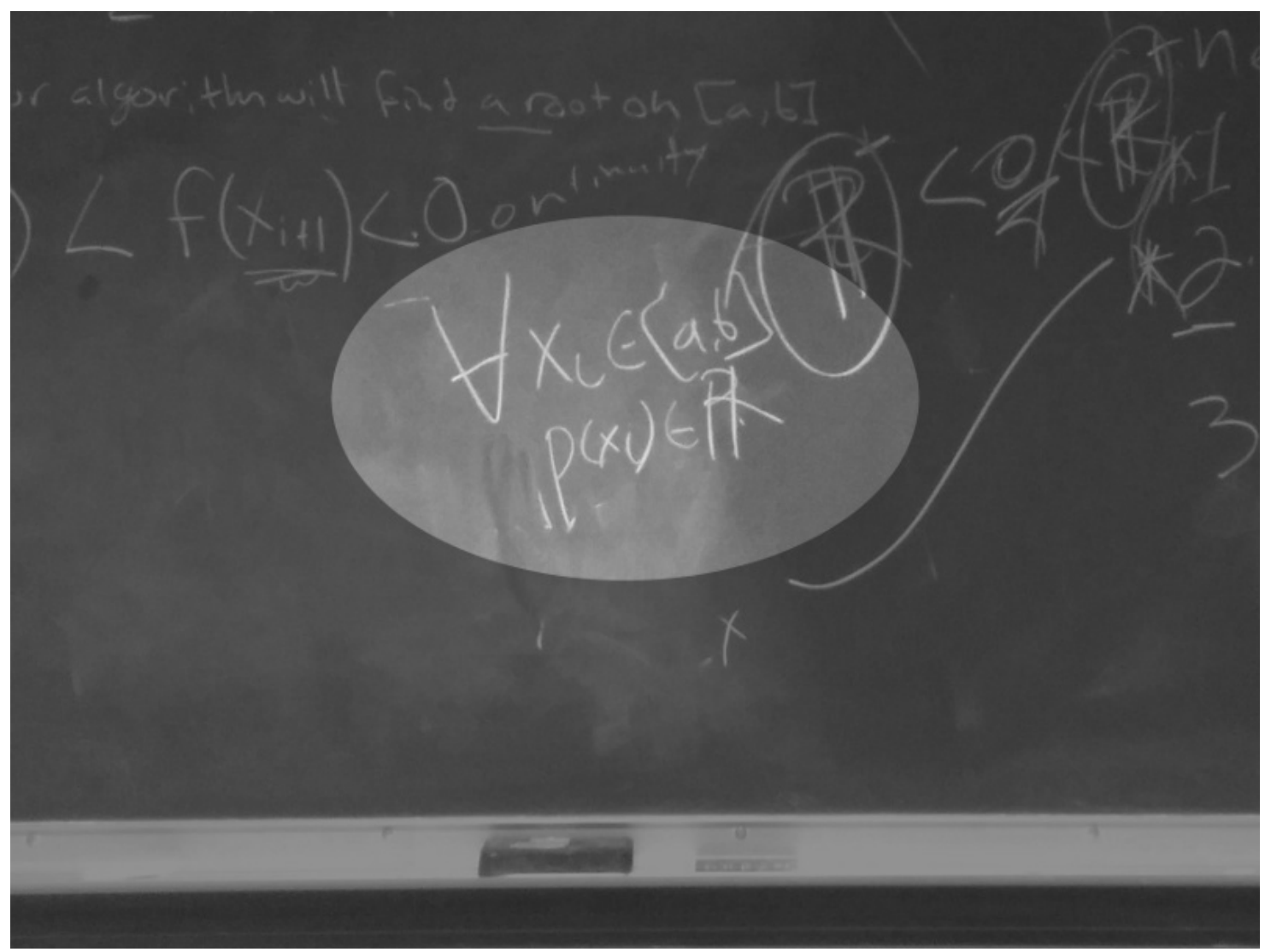

Figure 10: Dylan and Jay's first attempt at a formal definition of "continuous".

Evidently what they had written in mathematical notation was equivalent to: "for every point in the interval $[a, b]$, the function is defined." When I asked them if this captured continuity like they wanted it to, Dylan quickly recognized that, while it did eliminate holes or asymptotes, it did not eliminate jumping behavior. To illustrate this fact to Jay, Dylan sketched a step function, which satisfied their definition, but clearly was not continuous on the interval.

This conversation focused their efforts on trying to capture what was meant by a function having "no jumps". Dylan made reference to connected 
graphs from graph theory, but struggled to make an analogous statement for a function of the real numbers. It was then that Dylan verbalized an idea that struck me as anticipating an $\varepsilon-\delta$ characterization of continuity.

"On the $x$-axis- for every point on the $x$-axis, the point on the $y$-axis is also...like, the change is proportionate amounts? Like if you change $x$ a very small amount, like you have some $d x$, your $d y$ also can't be much larger, so you're not getting these gaps."

In that moment I did not have a good strategy for how to capitalize on this promising idea. And shortly after this the conversation turned to issues of convergence for their algorithm, and our focus shifted in that direction for the next few sessions. Ultimately we did not have time to return to this discussion in order to develop these ideas further.

\section{Summary of Phase 3}

Here in Phase 3 we saw the students describe their informal conceptions of continuity in trying to make sense of the IVT. In both cases, the students described a continuous function as one having "no jumps or breaks", but they also recalled that limit characterization: namely that the function is continuous at a point if the limit equals the function's value.

In the case of Dylan and Jay, we saw them take the first steps in attempting to formalize the idea that a continuous function should have no "jumps" or "breaks". This was the essential feature on which they focused to justify why the number approached by their sequence of approximations must be 
a root of the function. Though they were not able to construct a formal definition of continuity, Dylan's statement that "if you change $x$ a small amount...your $d y$ can't be much larger" could be modeled by the $\varepsilon-\delta$ definition of continuity. This suggested to me that such a definition might be a useful emergent model as students worked to develop a formal definition, and then used that definition to complete a proof of the IVT. I intend to test this hypothesis in future teaching experiments.

Having seen the most important developments through two teaching experiments, I will now present the most up-to-date version of the LIT. As with the instructional sequence, this LIT will be presented in three phases. As I mentioned in the introduction to the Results section, this structure was motivated by my analysis of the students' activity. It became clear that their algorithm could be used as an emergent model; each of the three phases roughly corresponds to the levels of activity (situational, referential, general) in which students engage with their approximation algorithm. Briefly, students begin at the level of situational activity as they develop an algorithm to approximate the root of a specific polynomial, using the principles underlying the IVT. Students transition to activity at the referential level as they investigate and seek to justify the behavior of their algorithm. Finally, students operate the general level as they use their algorithm to construct a proof of the IVT. Along the way we will see how students are supported in developing the concepts of sequence convergence, completeness, and continuity. 


\section{Proposed Local Instructional Theory}

In this section I will present a local instructional theory for using the Intermediate Value Theorem as a starting point and touchstone for multiple advanced calculus topics. I have refined this LIT through the course of implementing and analyzing the two teaching experiments detailed above. I will present this LIT in the paragraphs below as a generalized instructional sequence consisting of three phases. Briefly, in the first phase student activity consists of horizontal mathematizing, as they develop an algorithm to approximate the root of a continuous function. It is also in this first phase that students make a conjecture equivalent to the Intermediate Value Theorem. Student activity transitions to vertical mathematizing in the second phase, as the focus of study shifts to the algorithm itself. In this phase student strategies emerge that anticipate the formal concepts of both sequence convergence and completeness, as students work to justify their conjecture that their algorithm will find a given root. In the third and final phase, students work to use their algorithm and their newly constructed understandings as tools with which to prove the IVT as they have conjectured it.

Using the design heuristics of emergent models and didactic phenomenology, I will describe the key steps in each of the three Phases of the LIT below. 


\section{Phase 1: Approximating the Root}

Inspired by Cauchy's adaptation of a root-approximating algorithm into his proof of the IVT, the initial tasks should support students in developing such an algorithm. This algorithm will then serve as an emergent model through the subsequent phases of the LIT, as students analyze and investigate the algorithm, and then use it as a tool to complete a proof of the IVT.

Using the heuristic of didactic phenomenology, one problem that requires such an algorithm is approximating the root of a polynomial that changes sign over a given interval. For this reason student activity begins at the situational level, as they work to approximate the root of a given polynomial on a given interval. While there are many valid techniques for approximating roots (e.g., using secant lines, or Newton's method, etc.), it is important that the students develop an algorithm that generates a sequence ${ }^{9}$ that approaches the conjectured root. This is because the algorithm and the sequence(s) it generates will be the objects of study in Phase 2, in which students investigate the nature of sequence convergence and the completeness of the real numbers. This, in turn, will lay the foundation for Phase 3, in which students use their algorithm, and at least one of the sequences it generates, in constructing a proof of the IVT.

To put students in the mindset of thinking in terms of the IVT, the opening task of the LIT simply asks students whether or not a given polynomial has a root in the given interval ${ }^{10}$. Student justifications are based on the sign change of the

9 In fact, it is most probable that their approximation algorithm will generate a multitude of sequences. 10 Recall that in the second teaching experiment, students were given $x^{\wedge} 5-x-5$ on the interval $[0,2]$. 
function over the interval, as well as its assumed continuity. These justifications serve as the first conjecture of the IVT, and prepare the students to reason from the sign-change when subsequently constructing their algorithm.

Follow-up tasks have students approximate the root of a polynomial to specific, and increasingly demanding, measures of accuracy. While many such approximations are possible, breaking the interval into smaller pieces and checking for sign changes of the function is a powerful strategy. Such a strategy not only allows one to achieve a given degree of accuracy with certainty (as the task requires), but is also readily iterated to achieve any desired accuracy (as the follow-up tasks require). Didactic phenomenology suggests that such follow-up tasks might be useful in supporting students in constructing an algorithm that meets the desired requirements.

The concluding tasks of Phase 1 of the LIT serve as a bridge to the deductive Phase 2, in which students will begin vertical mathematization, as the algorithm itself becomes the object of study. One task that can initiate the transition to Phase 2 is to have the students identify and describe all of the possible sequences generated by their approximation algorithm. This is also a useful task to motivate a discussion about what a sequence is.

A number of different sequences arise from the students' approximation algorithms. One may consider approximations that approach the root in a monotonic fashion, either from the left or from the right. Similarly, reasoning from sign changes in the outputs, it is possible to construct a sequence of 
approximations that bounces around the root. Alternatively, one may also consider the intervals themselves as a sequence of sets, which converge to a set containing a single element: the root itself. Yet another sequence arises if one considers the lengths of the interval after each iteration as a bound on the error in each approximation. These error bounds form a sequence that monotonically approaches zero ${ }^{11}$. While student approximation algorithms may differ in structure and implementation, the most important artifacts for the students to record and analyze will be the resulting sequences.

This task has a number of potential benefits. First, it transitions the students to mathematizing their previous activity, by initiating the students' reflection on and investigation of their own algorithm. This vertical mathematization facilitates the development of their algorithm as a model-for more formal reasoning about the IVT by transitioning students to a more general level of mathematical activity. Second, it produces a set of artifacts (sequences) for further investigation. This investigation will serve as the primary activity in the second phase of the LIT.

\section{Phase 2: Justifying the Algorithm}

In this second phase of the LIT the emergent models design heuristic provides particularly powerful tools for describing student activity and development. In this phase student activity is at the referential level, as they refer

11 In the case of the Bisection Algorithm, this sequence was given by a simple algebraic formula: $\mid \mathrm{b}$ $\mathrm{a} \mid \mathrm{2}^{\wedge} \mathrm{n}$, while in the Decimal-Expansion Algorithm this formula would be: $|\mathrm{b}-\mathrm{a}| / 10^{\wedge} \mathrm{n}$. 
back to their root-finding activity and continue the process of mathematizing that activity. It is at this stage, through this process of progressive mathematizing, that their algorithm begins to transition from a model-of their activity to a model-for more formal reasoning about the IVT. This transition will be complete in the third phase, as student activity transitions to the general level and their approximation algorithm becomes a model-for completing their proof of the IVT.

The primary task of the second phase of the LIT is: How do you know that your algorithm will find a number? Note that students will likely talk about this number as if it is a root, even though proving that it is a root requires a formal definition of continuity. Using the heuristic of didactic phenomenology, the task of justifying that their algorithm finds a number will ultimately require students to formally define sequence convergence, and to articulate the completeness of the real numbers in some way. Student justifications at this point depend on the convergence of the sequence(s) generated by their approximation algorithm.

While a number of possible sequences emerge from the students' algorithm, their convergence necessarily depends on the completeness of the real numbers. This is due to the fact that the root-candidate is not known in advance; their sequence converges to a number presumed to exist due to the completeness of the real numbers. For this reason, one of the most important things for the students to clearly record is their own justification for why their sequence converges. For example, students will give justifications like "the widths of the intervals go to zero" or "the approximations are always increasing 
and never pass the root". These justifications amount to assertions of the completeness of the real numbers: in the first case, the fact that "widths going to zero" results in a unique real number is essentially the Nested Interval Property; in the second, the fact that such a sequence must necessarily converge is the Monotone Convergence Theorem. While the students' thinking about convergence is informal at this point, there are useful features that can be leveraged to support the development of more formal thinking. These features will be elaborated below.

The next step in vertical mathematization involves the students analyzing these justifications. To facilitate this, the students should work to codify these justifications as conjectures (e.g., "The intersection of a sequence of nested intervals whose length goes to zero contains a single element", or "a monotonically increasing sequence that is bounded above converges"). The task then is to prove these conjectures, which for the students is horizontal mathematizing at a new level of formality. In terms of didactic phenomenology, constructing proofs of these conjectures presents students with a need to formally define sequence convergence, and to develop some formal characterization of completeness. The students may not explicitly recognize this, but they will need to find a characterization of completeness which they can accept as an assumption in order to complete the proof. We saw this with Dylan and Jay when they agreed to assume the existence of least-upper bounds for bounded sets. 
For the proofs of some conjectures, students may only need to define a specific type of sequence convergence (e.g., monotonic convergence). To motivate a more general definition of convergence, consider other (carefully selected) sequences generated by their algorithm(s). In this context, having the students iteratively refine their definitions against such a set of examples (and non-examples) of convergent sequences has been shown to be particularly fruitful (Swinyard \& Larsen, 2012; Oehrtman, Swinyard, \& Martin, 2014).

The completion of their proof that their algorithm will find a given root marks the end of the second phase of the LIT. Rather than assuming the existence of a root, students will now be explicitly tasked with proving the root's existence under the conditions they have described. It is in this third phase that student activity moves to the general level, with respect to their algorithm, as this algorithm becomes a tool and model-for more formal reasoning about the IVT. Students will also work to develop their understanding of continuity in this third and final phase of the LIT.

\section{Phase 3: Proving Existence of the Root}

In the third phase of the LIT, the primary task is: Prove that given your conditions, namely that a continuous function changes sign on an interval, a root must exist. In terms of didactic phenomenology, this proof requires several tools. First, it requires students to re-purpose their algorithm: rather than using to find find a root, students now use it to provide a justification for the root's existence. 
This is a non-trivial shift, and in fact is one of Cauchy's major contributions to early real analysis (Grabiner, 1981). Doing so represents the final step in the transition of their algorithm into a model-for more formal reasoning about the IVT. Second, this proof requires students to develop a formal definition of what it means for a function to be continuous at a point. Below I will describe how continuity first emerges as a model-of students justifications for their conjectured IVT. Though this part of the LIT is currently the least developed, I will outline my hypotheses for how this model might develop into a model-for more formal reasoning about continuity and the IVT.

There are two primary presentations of the idea of continuity in most calculus courses. The first and most intuitive is that a continuous function is one that "can be drawn without removing your pen from the paper" (Stewart, 2003). (Note that for functions of the real numbers that are continuous on an interval this is accurate, though not particularly helpful in formal proofs.) The other presentation involves limits and goes something like this:

A function $f$ is continuous at a point $a$ if all of the following are true:

1. $f(a)$ exists

2. the limit as $x$ approaches a exists and

3. this limit is equal to $f(a)$

A function is then continuous on an interval provided that this condition is met for each point in the interval.

As students begin to construct a proof of the IVT, they articulate their 
conceptions of continuity, and to begin to reflect on how those conceptions might help explain the Intermediate Value Property. While students will likely remember one or both of the above characterizations from calculus, it will be their interpretations of these characterizations in the context of this root-approximation task that anticipate a formal characterization of continuity. In this way continuity emerges as a model-of their thinking. Depending on which of these interpretations is preferable, a choice can be made about which development path to follow. In either case, students should be supported in developing a formal definition of continuity, which will serve as a model-for more formal reasoning about continuity in the context of proving the IVT.

In constructing their proof of the IVT, students encounter, perhaps for the first time, questions that cause them to reflect on and consider more deeply their understanding of what it means for a function to be continuous. Using the heuristic of didactic phenomenology, it is this problem whose solution requires a formal definition of what it means for a function to be continuous at a given point. One possible path, as followed by Brad and Matt in the first teaching experiment, would be to reflect on and formalize the limit definition of continuity as encountered in a differential calculus class. Again using the heuristic of didactic phenomenology, this problem can be used further to motivate the need for a formal definition of the limit of a function at a point.

Alternatively, one may have the students begin with their informal notions of continuity ("able to be drawn without lifting the pen"). Recall from the second 
teaching experiment how Dylan attempted to capture this idea more formally:

"On the $x$-axis- for every point on the $x$-axis, the point on the $y$-axis is also...like, the change is proportionate amounts? Like if you change $x$ a very small amount, like you have some $d x$, your $d y$ also can't be much larger, so you're not getting these gaps."

From an instructional design perspective Dylan's statement shows promise as a starting point for developing continuity more formally. I will explain why this is a promising statement, what might have elicited it, and how it might be leveraged to support more formal student thinking. First, his statement can be loosely modeled by the $\varepsilon-\delta$ characterization of continuity, and as such it can be considered a strategy or idea that anticipates a formal conception of continuity. There are some important details to work out, not the least of which is the necessary switch to a range-first perspective (similar to that for formal limits, as proposed by Swinyard \& Larsen (2012)), but the idea of small changes in $x$ resulting in small changes in $y$ at least contains the core idea of formal continuity at a point. Second, this statement was prompted by considering the very informal notion of a continuous function having "no jumps". For this reason it seems likely that this task (of formalizing either what we mean by "no jumps" or possibly what would constitute a "jump") might elicit a similar statement from other students.

Finally, it seems plausible that this statement could be leveraged to support students in developing a formal definition of continuity. More research is needed to learn how to leverage this idea to develop a definition. As they reflect on their algorithm, other continuous functions, and perhaps their recent work in 
formalizing sequence convergence, they will construct such a definition for themselves. Then, as they work to complete the proof of the IVT, this definition will serve as a model for more formal reasoning about continuous functions and the IVT.

The third phase of the LIT concludes when students have successfully completed their proof of the IVT. Though likely time intensive, students have developed quite a bit through the course of this LIT. In broad strokes, through developing an approximation algorithm, analyzing and justifying that algorithm, and then using that algorithm to construct a formal proof of the IVT, students have developed more formal understandings of the topics of sequence convergence, completeness, and continuity. These are some of the core concepts in an advanced calculus course, and so this sets students on solid footing to explore further the theoretical underpinnings of the calculus.

I will now present an instructional sequence developed using this LIT. Like the LIT, this instructional sequence has been modified through the course of the two teaching experiments described above.

\section{Proposed Instructional Sequence}

In this section I will present an instructional sequence that aligns well with the LIT described above. First I will give a brief overview of each phase, and then I will present the tasks in outline form. 


\section{Phase 1: Approximating the Root of a Polynomial}

In this situational phase students develop an algorithm that allows them to approximate a root of a polynomial using the principles underlying the IVT. This algorithm will serve as the object of study in the second phase, and will support them in proving the IVT in Phase 3. The development of such an algorithm can be motivated by the following tasks:

- Task 1: Does $p(x)=x^{\wedge} 5-x-5$ have a root in the interval [0,2]? Justify your conclusion.

- Task 2: Approximate the root to two decimals places.

- Follow-up: Approximate the root to six decimal places.

- Follow-up: Can you approximate the root to any desired degree of accuracy? Justify.

- Task 3: Describe your algorithm for a general function $f(x)$ on an interval $[a, b]$.

- Follow-up: Under what conditions will your algorithm be guaranteed to find a root?

- Task 4: What are all the possible sequences generated by your algorithm?

\section{Phase 2: Justifying the Algorithm}

In this referential phase students engage in vertical mathematizing by reflecting on and organizing their previous activity. Here we see student strategies that anticipate the concept of completeness. These strategies can be codified into conjectures. By developing, analyzing, and refining a proof of one of these conjectures, students engage in horizontal mathematizing at a new, more formal level. Also through this process, students will need to develop a formal definition of sequence convergence and some form of the completeness axiom. The following tasks support this development: 
- Task 1: Assuming that a root exists, how do you know that your algorithm will find it? This will entail the students making claims about convergence which can be codified as conjectures. Informal notions of continuity may appear here; these should be recorded for further examination in Phase 3.

- Task 2: Prove [justification modified as conjecture]

- Follow-up: Formally define needed type of sequence convergence

\section{Phase 3: Proving the Existence of a Root}

In the third and final phase students engage in general activity with respect to their approximation algorithm. This algorithm becomes a model-for more formal reasoning and a tool for students to complete the first part of the proof of the IVT; namely, proving the existence of a root-candidate. It is in justifying that this root-candidate really is a root that continuity emerges as a model-of student strategies, and is developed into a model-for more formal reasoning about continuity in the context of proving the IVT. The development of this model is facilitated by the construction of a formal definition of continuity at a point, which students can then use to complete the proof of the IVT.

- Task 1: Prove that, under your conditions, a root is guaranteed to exist.

- Follow-up: Formally define what it means for a function to be continuous at a point.

\section{Conclusion}

The RME design heuristic of guided reinvention suggests that students begin their investigations in contexts that are "experientially real" for them; that is, contexts in which they have familiarity, intuition, and tools for reasoning. Given 
their experience with real functions in calculus, the Intermediate Value Theorem is just such a context. Within the context of approximating the root of a continuous function (that changes sign across an interval), the Intermediate Value Theorem is a theorem that post-calculus students are likely to conjecture. The RME design heuristic of didactic phenomenology suggests that problems and tasks be chosen whose solution requires the formal structures and tools we wish students to develop. The proof of the IVT presents students with a need to formally define such fundamental advanced calculus topics as sequence convergence and continuity, in addition to requiring them to wrestle with the concept of completeness for the first time. In accordance with the RME instructional design heuristics of guided reinvention and didactic phenomenology, this context is a promising one to motivate the development of formal understanding of sequence convergence, completeness, and continuity.

In this context, the students' approximation algorithm serves as the central emergent model in the LIT, motivating the three-phase structure corresponding to the levels of activity of the emergent models design heuristic: situational, referential, and general. As detailed in the LIT, this model also describes the process by which sequence convergence, completeness, and continuity are formally developed.

There is still much work to be done. The data so far suggest that the concepts of convergence and completeness are deeply intertwined, especially in the context of the IVT. A more in-depth exploration of how these concepts 
develop in tandem, and how they support one another, could greatly inform instructional design for advanced calculus and real analysis. For the first efforts in this direction, see Completeness and Sequence Convergence: Interdependent Development in the Context of Proving the Intermediate Value Theorem (Strand, in preparation).

Additionally, more data is needed regarding how students think about and engage with the larger goals of advanced calculus: namely, developing solid theoretical foundations for the calculus. In these experiments we saw students formulate important conjectures, and develop their own formal, powerful definitions for proving those conjectures. Future research should investigate more explicitly how students understand the process of developing foundations. A part of this process involves developing tools for formal proofs (e.g., formal definitions, using theorems and lemmas in larger proofs, etc.). Another aspect of this process involves choosing what are acceptable foundations. On a larger level this entails answering questions like, "What constitute acceptable proofs?" On a more specific level, with respect to completeness, this involves choosing a characterization that is acceptable without proof. (For Dylan and Jay in the second teaching experiment, this was the existence of least-upper bounds.) Explicit discussions with the students on these foundational ideas would shed light on when and how these topics should be addressed in the LIT.

Finally, far too little is known about how student conceptions of continuity grow and develop, which is one of the central goals of the third phase of the LIT. 
While this research has identified promising student strategies as starting points for formalizing continuity, further research will be needed detail and elaborate how these strategies can be leveraged to support the desired development. Also central to Phase 3 of the LIT is the students' re-purposing of their algorithm into a tool for proving existence. While to an observer the students' activity in Phase 2 could be construed as doing exactly this, there is little evidence that students conceived of their activity in this way. As this re-purposing of an approximation technique was one Cauchy's great insights and contributions, further research is needed to understand how students can be supported in understanding this fundamental shift in purpose. Future design experiments will investigate the third phase of the instructional sequence, and seek to inform further refinement of the LIT. 


\title{
Paper \#2 - Completeness and Sequence Convergence: Interdependent Development in the Context of Proving the Intermediate Value Theorem
}

\begin{abstract}
As a part of a larger RME-based instructional design project for advanced calculus, this paper reports on two students' reinventions of formal conceptions of sequence convergence and the completeness property of the real numbers in the context of developing a proof of the Intermediate Value Theorem (IVT). Over the course of ten, hour-long sessions I worked with two students in a clinical setting, as these students collaborated on a sequence of tasks designed to support them in producing a proof of the IVT. Along the way, these students conjectured and developed a proof of the Monotone Convergence Theorem. Through this development I found that student conceptions of completeness were based on the geometric representation of the real numbers as a number line, and that the development of formal conceptions of sequence convergence and completeness were inextricably intertwined.
\end{abstract}

\section{Introduction}

The transition from lower-division mathematics courses, where the emphasis is often on calculational approaches, to upper-division courses, primarily concerned with proof and more abstract mathematics, is a challenging one for many undergraduate students. There has been growing interest in developing research-based, student-centered curricula for undergraduate mathematics to address this issue in the areas of abstract algebra (TAAFU: 
Larsen, 2013; Larsen \& Lockwood, 2013), differential equations (IO-DE:

Rasmussen \& Kwon, 2007), geometry (Zandieh \& Rasmussen, 2010), and linear algebra (IOLA: Wawro, et al., 2012). The data presented in this paper comes from early efforts at similarly-motivated instructional design for advanced calculus. One of the central ideas underpinning all areas of advanced calculus is that of limits and convergence. One of the features of the real numbers that makes limits and convergence so important (indeed, possible) is that of completeness. While a large body of research exists about how students think about limits and how that thinking develops in formality, there is a dearth of research dealing directly with students' conceptions of the completeness of the real numbers.

This paper reports on the strategies employed by a pair of students that anticipated the concept of completeness, as those strategies emerged in the context of a teaching experiment. This experiment was part of an instructional design effort to develop the proof of the Intermediate Value Theorem (IVT) as a starting point for inquiry-oriented advanced calculus ${ }^{12}$. The students in the teaching experiment began by approximating the (irrational) root of a polynomial using the principles behind the IVT. They developed a sequence of approximations by looking for the sign change of the function on smaller and smaller intervals. As the IVT (and many other facets of convergence) implicitly depend on the completeness of the real numbers, I expected that investigations

12 see Paper \#1 
of these kind would give insight into students' informal conceptions of completeness, as well as insight into how students might be supported in reinventing formal characterizations of completeness.

In this paper I will detail how early student justifications anticipated the Monotone Convergence Theorem (MCT $)^{13}$, and how the proof of that theorem became a powerful context for the interdependent development of more formal conceptions of sequence convergence and completeness.

\section{Literature Review}

A great deal of research has investigated student understanding of the concept of limit. The focus of these investigations has shifted over the last few decades. Initially, a large number of studies sought to describe the difficulties students encountered when trying to work with limits (Bezuidenhout, 2001; Cornu, 1991; Davis \& Vinner, 1986; Moru, 2009; Sierpińska, 1987; Szydlik, 2000; Tall, 1980; Tall \& Schwarzenberger, 1978; Williams, 1991). Gradually, more and more studies have investigated how student conceptions might progress in formality (Cottrill, et al., 1996; Oehrtman, 2009; Oehrtman, Swinyard, \& Martin, 2014; Swinyard \& Larsen, 2012). One important feature of formal work with limits, first given prominence by Swinyard \& Larsen (2012), is the shift from finding limits to verifying limit candidates. While limit problems in calculus are often centered around the use of algebra to find limits, formal activity with limits is 13 MCT: If $\left\{a_{n}\right\}$ is a bounded, monotonic sequence, then it converges. 
usually centered around using formal definitions to prove that a limit exists or to prove general properties about limits. What has received almost no study is what the process of developing such formal definitions of limits looks like in the context of proving, or what role completeness plays in this process of formalization.

While Cauchy is widely recognized as one of the fathers of real analysis, his proofs conspicuously lack any mention of the completeness of the real numbers (Grabiner, 1981; Lützen, 2003). In fact, one of the first explicit treatments of the completeness ${ }^{14}$ of the real numbers was Dedekind's "Continuity and Irrational Number" essay, originally published in 1872 (1901), over fifty years after Cauchy's Cours d'Analyse. Up until Dedekind's time completeness, when it was discussed at all, was taken as a natural consequence of the geometric representation of the real numbers as a one-dimensional line (Dedekind, 1901). For these reasons it seems plausible that post-calculus students, who have a great deal of experience with the real number line, will treat completeness as an obvious property, when they think of it at all. However, it is a critical component of the proof of the Intermediate Value Theorem, and so identifying student strategies that anticipate formal conceptions of completeness in this context will be critical in developing instruction for advanced calculus using this context as a starting point.

As such, the specific questions that guided this component of this design research project were the following:

14 The German word that Dedekind used was the word for "continuity", but it is clear that he describing the modern concept of the completeness of the real numbers. 
1. In the context of proving the IVT, what student strategies anticipate the concept of completeness?

2. In what ways do the developments of completeness and sequence convergence support one another?

\section{Theoretical Framework}

The instructional design heuristics of RME have guided the development and implementation of this design project. They have also been indispensable as tools for analyzing student thinking and activity. In particular the heuristic of emergent models provides language and tools for describing students' activity at the informal level and also for describing the development of their activity toward greater formality and rigor. In RME, these models emerge from student activity, in the sense that the models provide a way for a teacher/researcher to describe student activity (Larsen \& Lockwood, 2013).

These models emerge as students engage in organizing some kind of problem context, also referred to as horizontal mathematizing. Led by the model, the teacher/researcher then tasks the students with reflecting on and organizing their own mathematical activity, a process known as vertical mathematizing. This then creates a new, more formal mathematical reality for horizontal mathematizing by the students. This process is known as progressive mathematization. Through progressive mathematizing, the model transitions from a model-of student activity to a model-for more formal reasoning on the part of the student. "This shift from model of to model for concurs with a shift in the students' thinking, from thinking about the modeled context situation to a focus 
on mathematical relations" (Gravemeijer, 1999, p. 162). In this way the design heuristic of emergent models provides ways not only to describe and make sense of student activity, but also to support students in reinventing the desired mathematics and in making the transition to more formal mathematical activity.

In emergent models, the model-of/model-for transition captures large, significant developments in student activity and thinking (Gravemeijer, 1999). For describing more local development of these models, Rasmussen and Marrongelle described the construct of a transformational record (2006). Such a record is an inscription or notation recorded by the students, or used by the teacher to capture student thinking, that later is used by the students for further mathematical development. This construct can be particularly useful for teachers in supporting the development of emergent models. In the Results section I will illustrate how I used a transformational record to support students in developing a more formal understanding of sequence convergence.

Transformational records can also be described using the RME construct of record-of/tool-for (Johnson, 2014; Larsen, 2004; Larsen, 2013). A record-of student activity generally refers to an inscription or notation that represents one form of the larger emergent model. This record-of becomes a tool-for when students use it for further mathematical development. This transformation of the record at a local level represents a development of the larger emergent model. A model-of students' activity can be evidenced by many different forms. For example, in the TAAFU curriculum students' initial activity working the 
symmetries of an equilateral triangle can be modeled by the group structure. This model takes on different forms at different times: a list of symmetries, an operation table, or set of rules for combining symmetries, etc. (Larsen, 2013). One way to describe the development of this model toward a model-for is through "students' increasing ability to reason with various forms of the model" (Johnson, 2014). Continuing with the previous example, when recording combinations of triangle symmetries, students construct an operation table; this table serves as a record-of student thinking about combining symmetries and is one form of the larger group structure as an emergent model. Such a table becomes a tool-for student reasoning as they work to develop a set of rules for combining symmetries, using the table to reason about patterns and relationships. While not as significant as a model-of/model-for transition, which represents the students becoming aware of and using the model as a whole, these record-of/tool-for transitions nonetheless represent important developments in student activity.

In the study reported here, we will see how the concept of completeness emerged as a global model-of student reasoning about the convergence of an approximation algorithm. Later least-upper bounds, as one form of that global model, emerged as a record-of student thinking. This form of the model then developed into a tool-for more formal activity as student used least-upper bounds in two important developments: 1) formally defining a specific mode of sequence convergence, and 2) completing a proof of the Monotone Convergence Theorem. 
After describing the structure and implementation of the teaching experiment, as well as the manner in which I analyzed the data, I will describe in detail how completeness emerged as a model-of student activity, and how that model developed through the course of the teaching experiment.

\section{Methods}

As a part of the early stages of an instructional design project, I ran two separate teaching experiments over the course of a year, a little more than six months apart. Each teaching experiment consisted of 10, hour-long sessions with myself as teacher/researcher and a pair of students working at a chalkboard at the front of the room. These students were volunteers selected from courses that were direct prerequisites to advanced calculus/elementary real analysis courses (e.g. Linear Algebra, Discrete Mathematics, Abstract Algebra, and Introduction to Proof), and who had expressed an intention to take advanced calculus in the near future. All four participants had completed the calculus sequence, differential equations, and at least one proofs-based course, prior to participating in the teaching experiment.

This data for this paper comes primarily from the second teaching experiment, with students who will hereafter be referred to as Dylan and Jay. With the first teaching experiment, my attention was primarily focused on issues of convergence. It was not until retrospective analysis that I discerned the 
importance of the role that completeness could play in this context. For this reason I modified the task sequence for the second teaching experiment, which resulted in an abundance of data relating to student understanding of completeness. For these reasons this paper focuses on the experiences of Dylan and Jay.

My main goal during ongoing analysis was to understand how students were thinking about the tasks in which they were engaging, as well as how they were thinking about the strategies they were employing. For this study I was particularly interested in how the concept of completeness was present in student thinking and justifications, and how that thinking might be leveraged and developed. To aid in this, I wrote session summaries and I kept a spreadsheet for each session, recording general student activities over the course of the session and marking segments for later transcription.

During the implementation of the teaching experiment there were anywhere from three days to an entire week between sessions. During that time I watched the videos of the previous session, creating written session summaries, and tried to identify student statements and strategies that begged for further investigation. For example, Dylan and Jay justified the convergence of a particular sequence by appealing to the fact that the sequence was increasing and was bounded. But it was not clear from their statements whether they thought such a sequence had to converge to the given bound. To start the next session I gave them exactly this conjecture and observed their discussion. 
After the conclusion of each of the teaching experiments, I performed a retrospective analysis of the data as a whole. I watched all of the videos again, transcribing segments I had flagged during the ongoing analysis, looking for student strategies that anticipated the completeness of the real numbers. For each of these I sought to explain what elicited these strategies. Finally, I followed these strategies through the data and using the design heuristics of RME I sought to explain how these strategies were leveraged to support the development of more formal ideas, or how they might be leveraged in future implementations of the LIT. For example, when describing a monotonic sequence converging to its bound, $b$, Dylan made a statement like "passes every a less than $b "$. It appeared that this strategy was elicited when Dylan tried to conjecture conditions under which such a sequence to converge to a given bound. This strategy then acted as a transformational record, when Dylan and Jay later used it as a tool to develop a formal definition of a sequence decreasing to zero. In the following section I will explain in detail how such anticipatory strategies emerged from Dylan and Jay's activity, and how these strategies were developed to support their construction of a proof of the Monotone Convergence theorem.

\section{Results}

In the context of developing their own proof of the IVT, I found that 
characterizations of the completeness of the real numbers emerged from Dylan and Jay's activity. This suggested to me that completeness, as a collection of varied but equivalent characterizations, could be seen as a model-of students' activity. While in this teaching experiment I did not see this model transition to a model-for more formal reasoning for the students, there were some significant developments. Using primarily the emergent models design heuristic, I will describe the students' progressive mathematization as they conjectured, and then worked to prove, the Monotone Convergence Theorem.

To begin the experiment, Dylan and Jay had asserted that $p(x)=x^{5}-x-5$ had a root in the interval $[0,2]$ because it was a continuous function that changed sign over the interval. This reasoning was essentially the Intermediate Value Theorem (IVT). Dylan and Jay subsequently constructed an algorithm that allowed them to approximate the conjectured root of $p(x)$ to any desired accuracy. Checking the sign of the function at values within the interval provided an expedient way for Dylan and Jay to narrow their search for the root: a point in the interior of the interval must evaluate to either positive, negative, or zero. If zero, then the search for a root is completed. If not, then the root must lie between sign changes of the function, and this gave them a smaller range to consider. Dylan and Jay identified successively more accurate decimal approximations to the root (e.g. 1.4, 1.45, 1.451, 1.4519,...) (Figure 11); this process amounted to a decimal expansion of the root. In this way Dylan and Jay constructed a sequence of approximations that monotonically increased toward 
the root, and which were bounded above by that root. That such a sequence must converge seemed intuitively obvious to them. This focus by the students on the monotonicity of their approximations served as the launching point for their investigations into completeness and convergence.

First Iteration:

\begin{tabular}{|c|c|c|c|c|c|c|}
\hline $\boldsymbol{x}$ & 1.41 & 1.42 & 1.43 & 1.44 & 1.45 & 1.46 \\
\hline$+/$ & - & - & - & - & - & + \\
\hline
\end{tabular}

Second Iteration:

\begin{tabular}{|c|c|c|}
\hline $\boldsymbol{x}$ & 1.451 & 1.452 \\
\hline$+/-$ & - & + \\
\hline
\end{tabular}

Third Iteration:

\begin{tabular}{|c|c|c|c|c|c|c|c|c|c|c|}
\hline $\boldsymbol{x}$ & 1.4511 & 1.4512 & 1.4513 & 1.4514 & 1.4515 & 1.4516 & 1.4517 & 1.4518 & 1.4519 & 1.4520 \\
\hline$+/-$ & - & - & - & - & - & - & - & - & - & + \\
\hline
\end{tabular}

Figure 11: The first three iterations of Dylan and Jay's Decimal-Expansion algorithm.

When justifying the convergence of their approximations Dylan and Jay made no arguments that relied on the widths of intervals, nor did they explicitly bound the error at a given iteration of their algorithm. Instead, their arguments tended to rely on the monotonic behavior of their approximations ${ }^{15}$. The transcript excerpt below came from a conversation in which Dylan, Jay, and I were discussing how they knew that their algorithm would find a root of the function in

15 Though they utilized ideas and notation that suggested sequences, Dylan and Jay did not themselves use sequence language until I asked them about sequences explicitly in the fourth session. 
question. They had recently established that, if the root were irrational, their

Decimal-Expansion algorithm would never give them the exact root.

I: So how do you know that there is such a number?

D: As long as we can recursively show that every time we step our function forward it gets a little bit closer to zero. This is how you do the limit in general: every time you step it forward, every time you know you move forward a little bit, you get closer to the number you think the limit is.

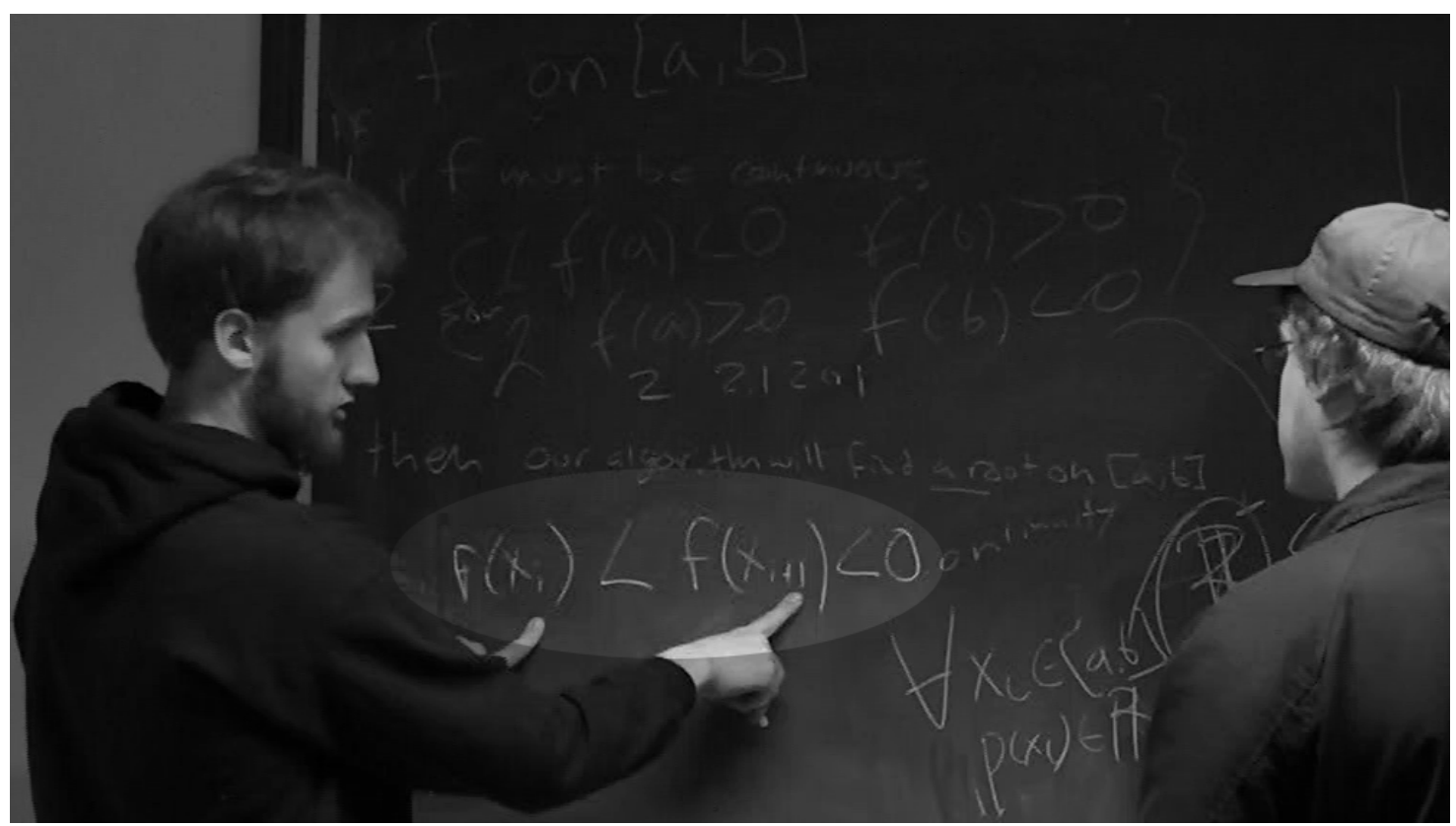

Figure 12: Dylan's justification for the convergence of their sequence of approximations.

There are a few problems with Dylan's characterization of a "limit in general". For one, he is a describing convergence in a monotonic fashion, and so is not truly giving a general description. Second, as can be seen in Figure 12, he is characterizing the convergence of their sequence of approximations using the 
monotonicity of the outputs of the function. This held true of the specific polynomial with which their investigations had started, but it was not necessary in general for their algorithm to work.

While there were many details to be worked out, Dylan's statement represented very promising reasoning. Here we see that the concept of completeness, here taking the form of the Monotone Convergence Theorem, served as a model-of he and Jay's explicit justifications for the convergence of their sequence of approximations. More specifically, his statement suggested that he believed an increasing sequence, that was bounded above, should converge. Whether or not he really believed that this characterized limits in general was immaterial at that moment. This emergent model suggested that codifying and analyzing Dylan's justification could be very fruitful.

In Figure 12, Dylan had written:

$$
f\left(x_{i}\right)<f\left(x_{i+1}\right)<0
$$

In an attempt to draw their attention away from the outputs for a moment, I asked Dylan and Jay whether a similar statement could be made about the inputs. I did this because I wanted to have them analyze Dylan's statement, but I did not want considerations about the behavior of the function to muddy the water. Without any discussion, Dylan wrote:

$$
x_{i}<x_{i+1}<\bar{x}
$$

(where $\bar{x}$ was the conjectured root). Then he and Jay explained why the second compound inequality might be preferable. 
Jay: We're controlling this [gestures at (2)] more than we're controlling this [gestures at (1)]. We can't control the outputs, but we can control the inputs.

Dylan: Right. I guess we just observe this [gestures at (1)] for this particular function.

With Dylan and Jay in agreement with the statement about the monotonicity and boundedness of the inputs, we were ready to consider their justification as a conjecture. Completeness, manifested as the Monotone Convergence Theorem, was an even clearer model-of their thinking about the convergence of their sequence of approximations. In order to support the development of their thinking, I set Dylan and Jay tasks that would have them engage in vertical mathematizing, by having them reflect on and analyze their own reasoning about convergence.

At this point, it was not clear to me whether they thought that a monotone sequence with a known bound had to converge to that bound. In an attempt to better understand their thinking, I offered them the following conjecture:

$$
\text { If } x_{i}<x_{i+1}<b \text {, then the sequence converges to } b \text {. }
$$

In a larger sense, what I was doing with this sequence of tasks was working to develop completeness as a useful model for the students. I was giving them tasks that caused them to reflect on both their own activity (specifically their algorithm) and on their own thinking, by presenting them with their justification codified as a conjecture. This vertical mathematization was the first step in supporting the transition of completeness from a model-of Dylan and Jay's 
thinking to a model-for more formal reasoning. While the model-for transition was not realized in the teaching experiment, the development of the model provided insight into the students' thinking about both completeness and convergence, as we will see.

After a few moments of individual thinking time, Jay concluded that such a sequence need not converge to just any bound. He wrote

$$
x_{i}<x_{i+1}<1000
$$

and then explained:

Jay: $\quad$ My thing was, uh, you could have $x \_i$ less than $x \_\{i+1\}$ less than, let's say, a thousand. But this sequence, it doesn't necessarily have to converge to a thousand. This would be satisfied if it converged to two.

Dylan: Yeah, that was my first problem: it doesn't necessarily converge to $b$. What I think is interesting is that this does mean that it necessarily converges. That it never passes some value.

Dylan agreed with Jay's reasoning, but made the additional observation that, given these hypotheses, the sequence must converge to something. He then went on to explain his thinking further, and to conjecture some conditions for when the sequence would converge to $b$.

"Because if you can pick a value, some $a$, between $x_{i+1}$ and $b \ldots$ and ... $x_{i+1}$ passes every value of $a$...like every possible value of $a$...and passes $b$...wait, if this is true, so it doesn't pass $b$. So worst case scenario it converges to $b . "$

It appears that what Dylan described was essentially a characterization of $b$ as the least-upper bound of the sequence. He seems to suggest that if we 
could choose a to be any arbitrary value less than $b$, and then we knew that a value of the sequence $\left\{x_{i}\right\}$ passed that value of $a$, then the sequence would have to converge to $b$. So there was no value of $a$ less than $b$ that was also an upper bound for the sequence.

This condition, that the sequence passes every value of a less than $b$, but never passes $b$, proved to be pivotal in Dylan and Jay's developments of both completeness and convergence. A short while after this, I incorporated this condition as an added hypothesis to their MCT and had them consider it; in this way it became a record-of their thinking, and also represented one form of the larger completeness model. Subsequent to that discussion Dylan explicitly leveraged the condition to define "decreases to zero". Both of these developments will be discussed in greater detail shortly.

Though Dylan and Jay briefly attempted to prove their MCT, they quickly realized that they would need a more precise definition of convergence in order to do so. Their first attempts at defining monotonic convergence relied on the fact that the successive differences must be decreasing. But Dylan recognized that this was not sufficient; even if the successive differences decreased, the sequence might still diverge to infinity (he cited the Harmonic series as an example of this).

Dylan: How do we better define when we know a sequence is going to approach a number? Because literally the counter-example is, well, this is $1 / i$.

Jay: The distance?

Dylan: Yeah. This distance every time is $1 / i$, and this will keep adding 'til we 
add up to infinity, for whatever reason. But we know, like, $1 / i^{\wedge} 2$ doesn't...What's the difference?

Though this consideration of successive differences was reminiscent of Cauchy's criterion for convergence, Dylan and Jay were unable to identify conditions that would guarantee convergence at that point in time. They were very clearly stuck.

In an effort to help them, I suggested a different approach. I asked them to try and define what it would mean for a general sequence to converge, rather than a monotonic one. Of their own accord they began considering different examples of convergent sequences. Though they briefly considered monotonic sequences that increased or decreased toward a limit, ultimately they settled on the damped sine curve as their prototypical example. They agreed that such a sequence converged, and so they set about trying to characterize that convergence. 


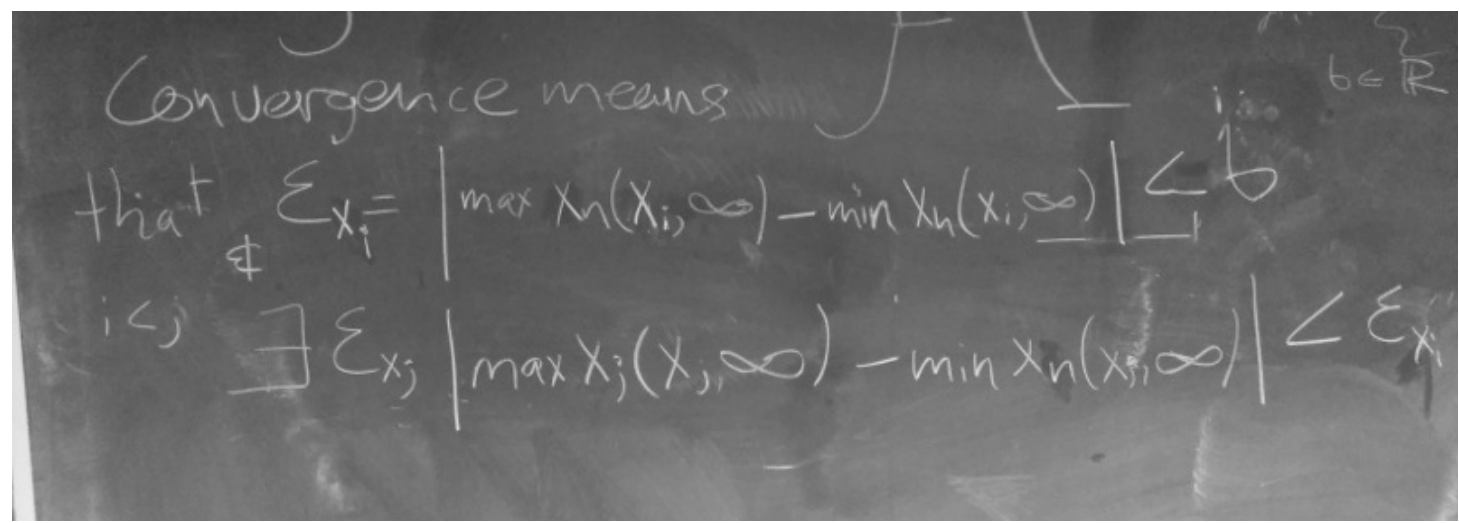

Figure 13: Dylan and Jay's first attempt at defining sequence convergence.

Dylan and Jay first characterized general sequence convergence by considering the "error", which they defined as the difference between the maximum value of the sequence and the minimum value of the sequence, from some point on (Figure 13).

"So this $\varepsilon$ is...let's just define $\varepsilon$ of $x_{i}$ is the biggest value the sequence- the difference between the biggest and smallest value the sequence takes from $x_{i}$ forward. Does that sound fair?"

Dylan and Jay then defined the convergence of the sequence $\left\{x_{n}\right\}$ as occurring when this sequence of errors decreased.

This, of course, is not quite sufficient, for a few reasons. First, the "errors" must decrease to zero to guarantee convergence. This error term can be thought of as a measure of the sequence's oscillation (though Dylan and Jay did not refer to it this way). If it decreased toward a non-zero constant, then the sequence would forever bounce back and forth between two constant values. Dylan and Jay recognized these difficulties, as evidenced by this exchange: 
Dylan: So could [the errors] converge to, say, 1 ? Then [the original sequence] would converge to a shift of the sine curve. So...does [the sequence of errors] need to converge to zero? Because a function like that doesn't.

Jay: If these errors are always- the errors are getting closer and closer to one? You're not- the actual sequence isn't converging to anything. It's still oscillating.

Dylan: Right. So that is a problem.

Jay: So there's a limit involved.

Dylan: Yeah, it has to go to zero. Period.

Second, Dylan and Jay's use of "max" appeared problematic because there are many sequences for which no "max" exists. For example, consider $x_{n}=$ $3 n /(n+1)$, which monotonically increases toward 3 without having a maximal element. However, it became apparent later that Dylan and Jay were using "max" in a way that could mean "max" (biggest element of a set) or could mean "supremum" (least-upper bound of a set) in standard terminology. Whether or not they believed at this point that all sequences had maximal and minimal elements cannot be determined from this evidence.

Finally, their use of indices was a bit problematic as well. Note that throughout their definition they have indexed by " $x_{i}$ ", rather than by " $i$ ". This was problematic because it suggested that they were indexing by the outputs of the sequence, when their sketches, gestures, and discussion made clear that they meant to index by the inputs. One possible explanation for this choice of index was that the sequence $\left\{x_{i}\right\}$ originally represented a sequence of values on the $x$ axis. In the interest of time I chose not to address this issue directly. The next time we referenced this definition I wrote it on the board with the correct indexing 
and the students used it correctly from that point on.

In the teaching experiment these issues served as opportunities for investigation and formalization of further ideas. In particular, reflecting on this definition of convergence in terms of "errors" motivated a number of other developments. The requirement that the "errors" decrease to zero motivated the development of a formal definition of what it meant for a sequence to "decrease to zero". This definition of convergence in terms of "max" and "min" values of the sequence from some point on motivated a clarification of the concepts of "max" versus "supremum", and the introduction of the terminology for "supremum" and "infimum".

Interestingly, this construct of "the sup from some point on" anticipates the idea of a "lim sup" or "limit supremum". In this way their definition of convergence could be seen as a strategy that anticipated the concept of a limit supremum, and potentially could be leveraged to support the development of the formal concept of limit supremum. However, from an instructional design perspective, I do not know how to elicit this very complicated characterization of completeness on purpose. For this reason I did not follow up with this particular potential development.

In a brief interlude, I returned our attention to the statement we were trying to prove: their conjectured MCT. Recalling Dylan's statement earlier about the sequence "passing every a less than b", I presented them with a modified version of their conjecture. I then asked them if this added condition would guarantee 
convergence to the bound, $b$ (Figure 14). This was an intentional move to further refine the emergent model of completeness. Implicit in this statement is the fact that $b$ is the least upper bound of the sequence. By having the students reflect on and make sense of this property, I hoped to support the development of completeness as an emergent model. I anticipated that this non-standard characterization of least-upper bounds would help them with their proof, and that completeness, possibly manifested as the existence of least-upper bounds, would in this way become a tool-for more formal reasoning about completeness and convergence. In this way, my codifying Dylan's earlier statement about "passing every a less than $b$ " was setting up a potential transformational record. This also fit nicely with the heuristic of didactic phenomenology, as the resolution of this proof would require the students to use least-upper bounds and a formal definition of convergence.

This record-of their thinking supported their development of sequence convergence in ways that I did not anticipate. This transformation will be evidenced when we consider how the students defined what it meant for a sequence to decrease to zero. 


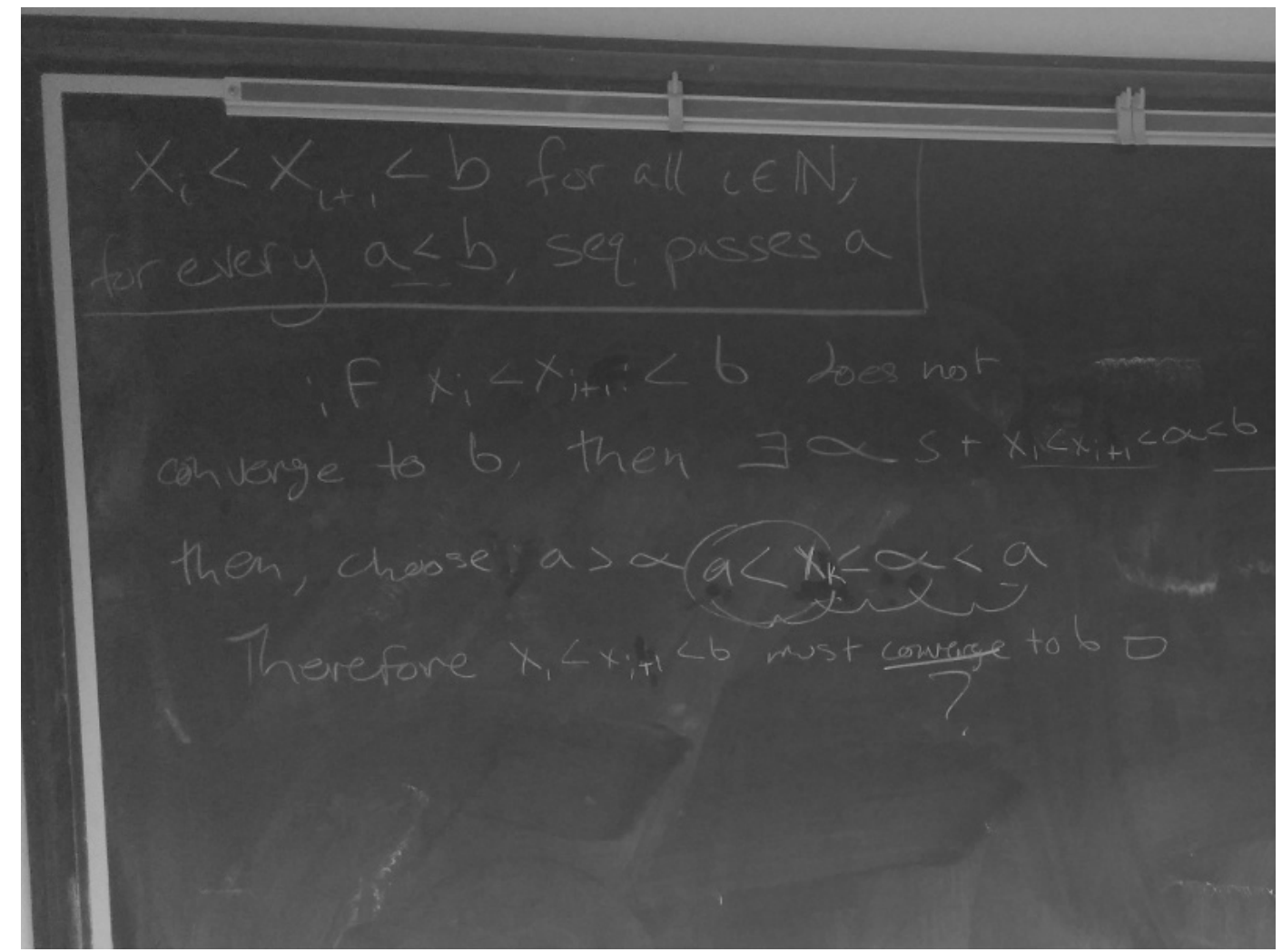

Figure 14: Dylan and Jay's first proof of a modified MCT.

Statement: $x_{i}<x_{i+1}<b$ for all $i \in \mathbb{N}$, for every $a \leq b$, seq. passes $a$.

If $x_{i}<x_{i+1}<b$ does not converge to $b$, then $\exists \alpha$ s.t. $x_{i}<x_{i+1}<\alpha<b$.

Then choose $a>\alpha \quad a<x_{k}<\alpha<a$.

Therefore $x_{i}<x_{i+1}<b$ must $\frac{\text { converge }}{?}$ to $b$.

Figure 15: The students' argument reproduced for legibility. 
Dylan and Jay agreed that this extra condition should guarantee convergence to $b$, and then set about trying to prove this. They argued that if the sequence did not converge to $b$, then there must be some smaller upper bound for the sequence, alpha. But since the sequence passed every a that was less than $b$, this resulted in a contradiction. Their technique was sound; all they needed was a formal definition of "converge" to make the proof rigorous. This motivated our return to the task of refining and clarifying their formal definition of sequence convergence.

At that point I opted to share with Dylan and Jay that their characterization, though non-standard, was one way to define the least upper bound of a sequence.

"So this condition that you guys came up with actually has a name. And if a number satisfies this condition- there are different ways to say it, but $b$ in this case is called the least upper bound."

After a brief discussion of this concept, Dylan summarized his understanding in this way:

"You can pick any number bigger than $b$, and this inequality would also be true. But you can't pick a number smaller than $b . "$

The discussion of this concept would resurface when I asked the students to define the "max" of a sequence, as used in their definition of convergence.

We then returned to their characterization of convergence in terms of "errors". After a brief discussion clarifying that convergence could only happen in 


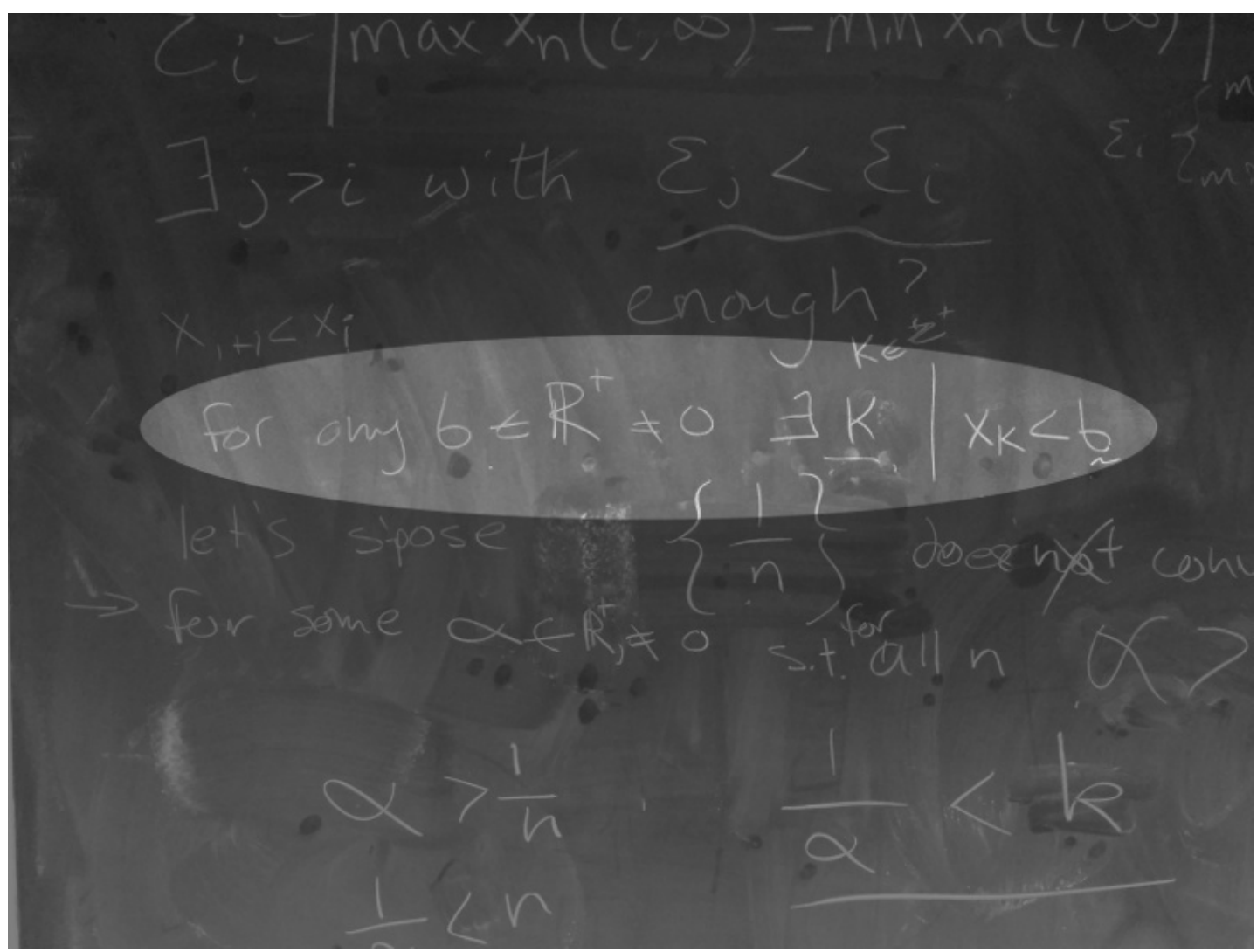

Figure 16: Students' definition of a sequence "decreasing to zero"

the case the $\varepsilon_{i}^{\prime}$ 's decreased to zero, we set about formally defining what it meant for a sequence to "decrease to zero". Recall that earlier, Dylan and Jay had struggled to define monotonic convergence, in particular because they had been considering successive differences. Remarkably, Dylan generated the pictured definition on the first try (Figure 16).

In the following exchange Dylan explained the genesis of this definition.

Jay: How'd you get that?

Dylan: Basically going from our last idea that if a number converges to...to $b$, I guess. So, this would be kind of like our- or this is kind of formally writing out that $a$, like, for every $b$ that's less than $a$, or- which is zero in this case, I guess- is between where we're starting and the 
boundary.

Dylan made a connection back to their work with a previous conjecture: that if a monotonically increasing sequence was bounded by $b$, but passed every a less than $b$, then it must converge to $b$. Though the roles of the variables have been reversed, Dylan has described adapting their idea of "passes every a less than $b$ " to this case of a sequence monotonically decreasing to zero.

Dylan goes on to explain his definition in more detail.

"So the boundary we know we want is zero. So we're going to talk about all the numbers that aren't zero, above zero. So these numbers [gestures at his definition, "positive reals not equal to zero"]...So we know this is always getting smaller. Down to some...you know, whatever. It goes off to somewhere. But do you know it goes to zero? And you do as long as you can pick any of these numbers [positive real numbers] and just keep going through until you find some $k$ [sic] that's smaller than it."

Here we see the results of the students successfully leveraging a transformational record. Earlier in the experiment, when reasoning about the conditions under which a monotonic sequence might converge to its bound, Dylan's made the statement "passes every a less than b". A little later I recorded this reasoning, presenting it back to the students as an additional hypothesis to their MCT; in this way this characterization of least-upper bounds served as a record-of their thinking. And above we saw how this record became a tool-for solving the problem of defining the convergence of a sequence decreasing to zero. In this way an informal strategy of the students developed into a tool-for reasoning more formally about limits. More specifically, my presentation of their 
strategy re-packaged as a conjecture acted as a transformational record, which they used to solve the problem of defining a sequence decreasing to zero.

The final step in clarifying their definition was to better understand how they were using the terms "max" and "min". To do this, I simply asked them to define the word "maximum", in the context of their definition. Jay defined it as the "least-upper bound", and illustrated this idea with a sketch of a sequence monotonically increasing toward its limit (Figure 17).

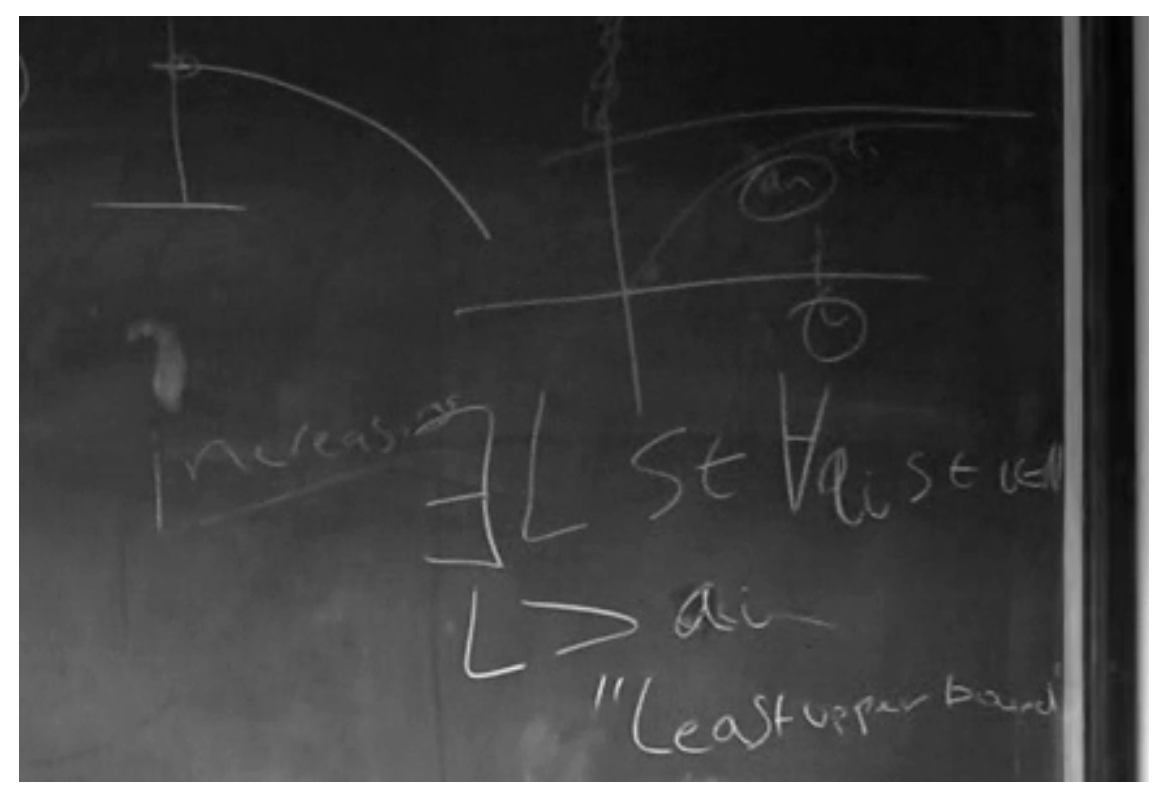

Figure 17: Jay's first attempt at defining "maximum".

Initially I thought that Dylan and Jay's use of "max" in their definition of convergence represented an error (since the "max" of sequence need not exist, in general). However, it became apparent that the problem Dylan and Jay faced was a lack of standard vocabulary to express their very robust conception of "max". 
Jay's initial inscription (Figure 17) clearly captures the idea that $L$ should be an upper bound for the sequence $\left\{a_{i}\right\}$. What is not clear, at least from the formal mathematical notation, is that this $L$ should be the least of these upper bounds. Also, Jay's use of a strict inequality precludes the possibility of the "least-upper bound" being a member of the sequence (and so a "max" in the standard sense).

The subsequent discussion made clear that these were problems of which Dylan and Jay were well aware, and wanted to solve. (Italics represent the student's emphasis.)

Dylan: Yeah, 'cause I think there needs to be a condition that it's the least one-

Jay: Right.

Dylan: And also, what if it's- what if you have a known maximum? That's where I kind of stopped myself. So let's say it's decreasing. So let's say it starts here, and then goes down... So this is the maximum. It's not the number that's very slightly greater than that. It's actually that number.

Dylan uses an example of a monotonically decreasing sequence, whose maximum would clearly be its first element, to illustrate a shortcoming in their definition. In the process of recording on the board the substance of their discussion, Dylan and Jay actually solved both of these problems (Figure 18). 


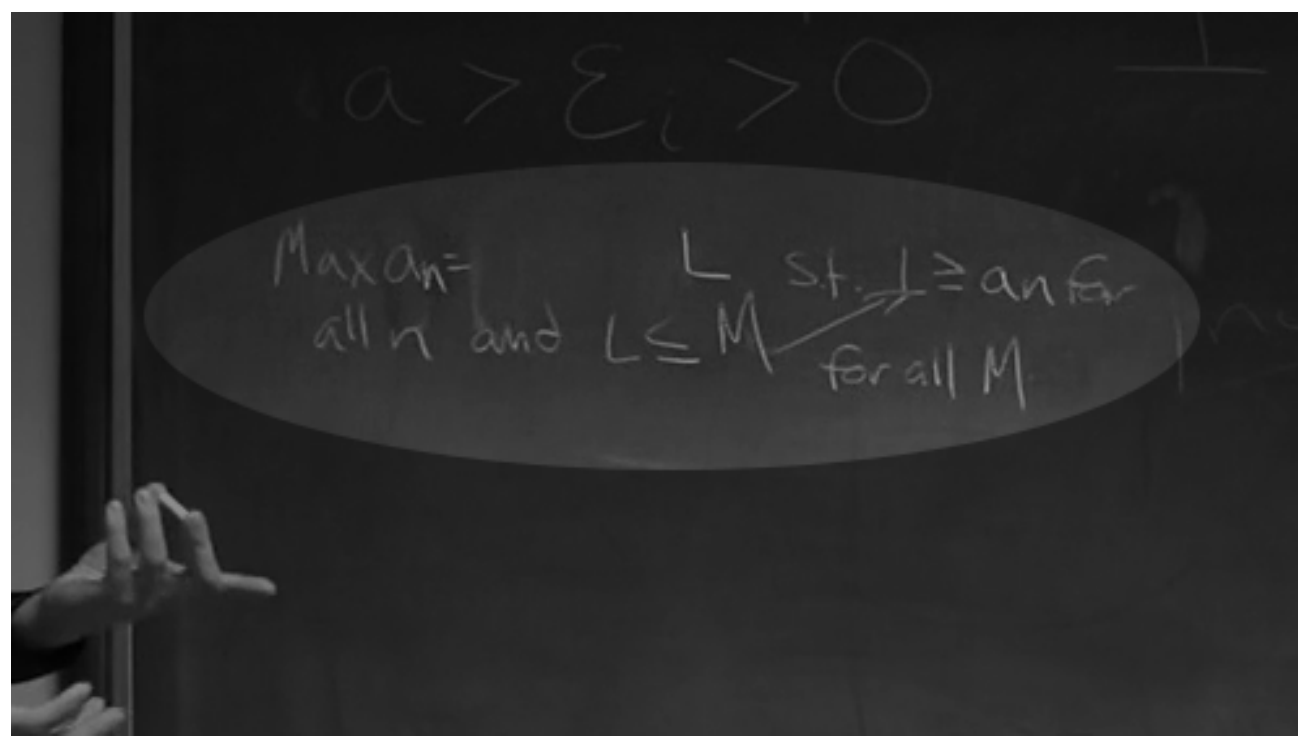

Figure 18: Dylan and Jay's refined definition of the "maximum" of a sequence.

Notice that in this new statement, $L$ is allowed to be greater than or equal to all the elements of the sequence $a_{n}$; this allowed for the fact that the "maximum" might be an element of the sequence. Also note the arrow, which points from $M$ toward the first inequality, $L \geq a_{n}$. This arrow was used to indicate that $M$ should also satisfy this inequality; that is, $M$ stood in the place of all other upper bounds of the sequence. In this way they have given the two conditions for what is commonly called the supremum of a set (here stated specifically for a sequence): that $L$ be an upper bound for the set, and that if there were another upper bound, $M$, then $L$ must be less than or equal to $M$. So when they used the word "max" in their definition of convergence, evidently what they were using was the concept of a "least-upper bound" in the standard sense. Whether this was Dylan and Jay's original intent with their definition, or whether this was something they only realized upon focused reflection, I do not have the data to determine. 
Here my motivations as a researcher were two-fold. I wanted to better understand their thinking, and how they were using the term "maximum" in this context. But I also recognized that completeness, manifested as the existence of least-upper bounds, would be a powerful tool in helping them finish their proof of the MCT. The task of defining "maximum" served as vertical mathematization: the students reflected on their own activity, and attempted to capture this thinking in a more formal definition. This provided students explicit access to least-upper bounds, further developing completeness as a global emergent model. 


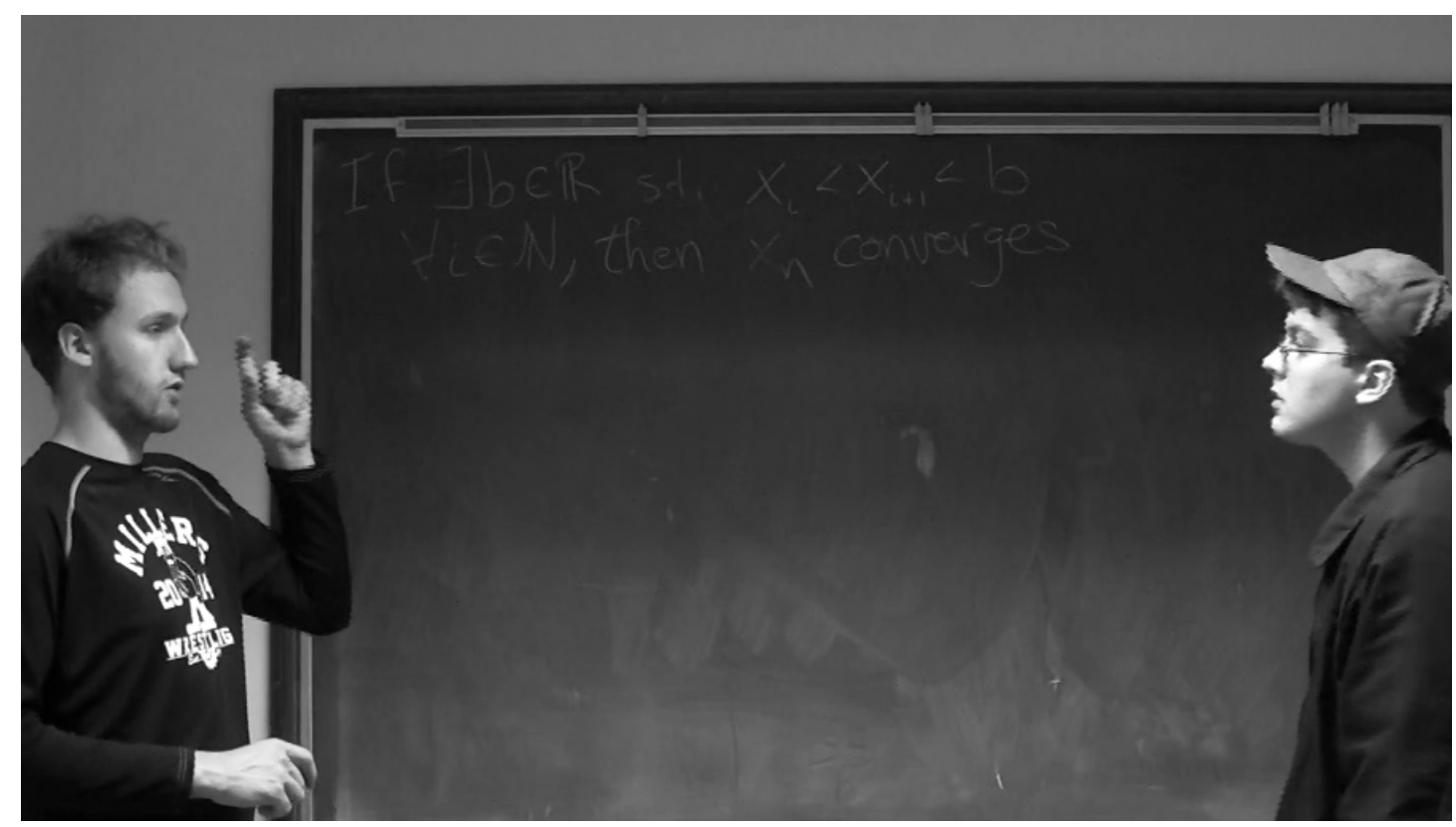

Figure 19: Statement of the students' conjectured MCT.

Once they were satisfied that their definition of "maximum" captured what they intended, we had a brief discussion in which I shared the standard terminology ("supremum" and "infimum") with them. From that point on Dylan and Jay used "sup" and "inf" in their definition of convergence. With a formal definition of convergence and a more explicit understanding of supremum/infimum, they returned to the task of proving their Monotone Convergence Theorem (Figure 19).

Jay determined that the first step in showing that the sequence, $\left\{x_{n}\right\}$ converged involved showing that the sequence of "errors" for the sequence $\left\{x_{n}\right\}$, denoted $\varepsilon_{i}$, was indeed decreasing. In fact, once Jay had successfully shown this he thought that this was sufficient to prove convergence. 


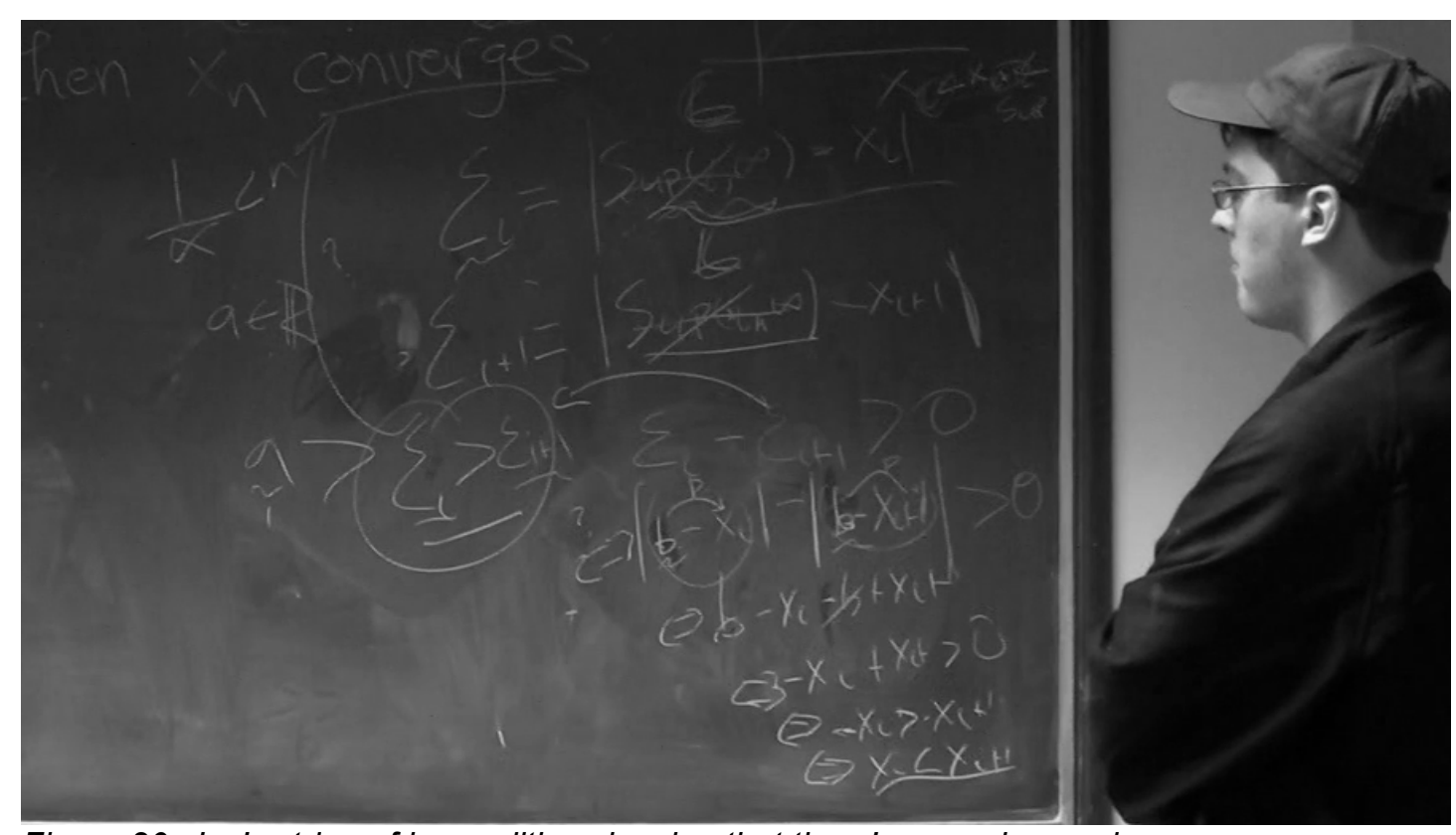

Figure 20: Jay's string of inequalities showing that the $\varepsilon_{i}$ 's were decreasing.

Jay's Proof that the $\varepsilon_{i}$ 's were decreasing

$$
\begin{gathered}
\varepsilon_{i}=\left|\sup (i, \infty)-x_{i}\right| \\
\varepsilon_{i+1}=\left|\sup (i, \infty)-x_{i+1}\right| \\
\varepsilon_{i}>\varepsilon_{i+1} \\
\Longleftrightarrow \varepsilon_{i}-\varepsilon_{i+1}>0 \\
\Longleftrightarrow\left|b-x_{i}\right|-\left|b-x_{i+1}\right|>0 \\
\Longleftrightarrow \not b-x_{i}-\not b+x_{i+1}>0 \\
\Longleftrightarrow-x_{i}+x_{i+1}>0 \\
\Longleftrightarrow-x_{i}>-x_{i+1} \\
\Longleftrightarrow x_{i}<x_{i+1} \checkmark
\end{gathered}
$$

Figure 21: Jay's string of inequalities reproduced for legibility. 
Jay began the proof by writing out the elements $\varepsilon_{i}$ and $\varepsilon_{i+1}$ for the monotonically increasing sequence $\left\{x_{n}\right\}$ given in the statement of the theorem. Note that since the sequence $\left\{x_{n}\right\}$ is monotonically increasing, the infimum from $i$ on will always be the current element, $x_{i}$. Though Jay would later protest the assumption of a single supremum for the whole sequence $\left\{x_{n}\right\}$, here he followed Dylan's suggestion and changed the "sup $(i, \infty)$ " statement to simply $b$. Though this seems an odd choice from the outside, given that the upper bound in the hypotheses was called $b$, both Dylan and Jay recognized that the value would cancel, whatever it was, and so were unconcerned with its actual designation. In Figure 21 Jay's proof that the sequence $\left\{\varepsilon_{i}\right\}$ (their sequence of "errors") was decreasing consisted of a string seven inequalities. Jay began with line (1), which was what he wanted to show. He proceeded to algebraically simplify and cancel, until he arrived a statement he knew to be true; namely, that the sequence $\left\{x_{n}\right\}$ was monotonically increasing (line (7)). Jay then went back and verified that the logic was valid in the reverse direction; that is, that he could start with line (7) and work his way back to line (1). He indicated this validity by prefixing each line with the double-implication arrows. Having satisfied both himself and Dylan that the proof was correct, he concluded that the sequence $\left\{\varepsilon_{i}\right\}$ was indeed decreasing, and that therefore the sequence $\left\{x_{n}\right\}$ converged. To conclude that $\left\{x_{n}\right\}$ converged, it was actually necessary to show that the sequence $\left\{\varepsilon_{i}\right\}$ converged to zero. Dylan and Jay would get to that shortly. Jay's proof that the sequence $\left\{\varepsilon_{i}\right\}$ was decreasing appeared to depend on 
two specific (and related) features of the sequence $\left\{x_{n}\right\}$ : that $\left\{\sup x_{n}(i, \infty)\right\}_{i}$ was constant, and that $\left\{x_{n}\right\}$ was monotonically increasing. However, it turns out that neither of these conditions are necessary, and that the sequence $\left\{\varepsilon_{i}\right\}$ will be decreasing for any sequence (for which it is defined). To see why this must be so, consider the two primary components of $\varepsilon_{i}$ : sup $x_{n}(i, \infty)$ and inf $x_{n}(i, \infty)$. There are only two possibilities for the sequence $\left\{\sup x_{n}(i, \infty)\right\} ;$ : either it is constant (some real number, or possibly infinity) or it is monotonically decreasing. To see this, consider the sequence in Figure 22. Since we are choosing the supremum (least upper bound) from some point on, it is clear that this value can never increase; if it did, that would simply mean that we did not choose the proper supremum at a previous step. Since it cannot increase, it follows that it must be constant or monotonically decreasing ${ }^{16}$

16 A sequence is decreasing provided that $j>i$ implies $x_{j} \leq x_{i}$, so that technically a constant sequence is monotonically decreasing. However, my intention is to distinguish between the case where the sequence is always constant and when it is only occasionally constant. 


\section{Damped Oscillation}

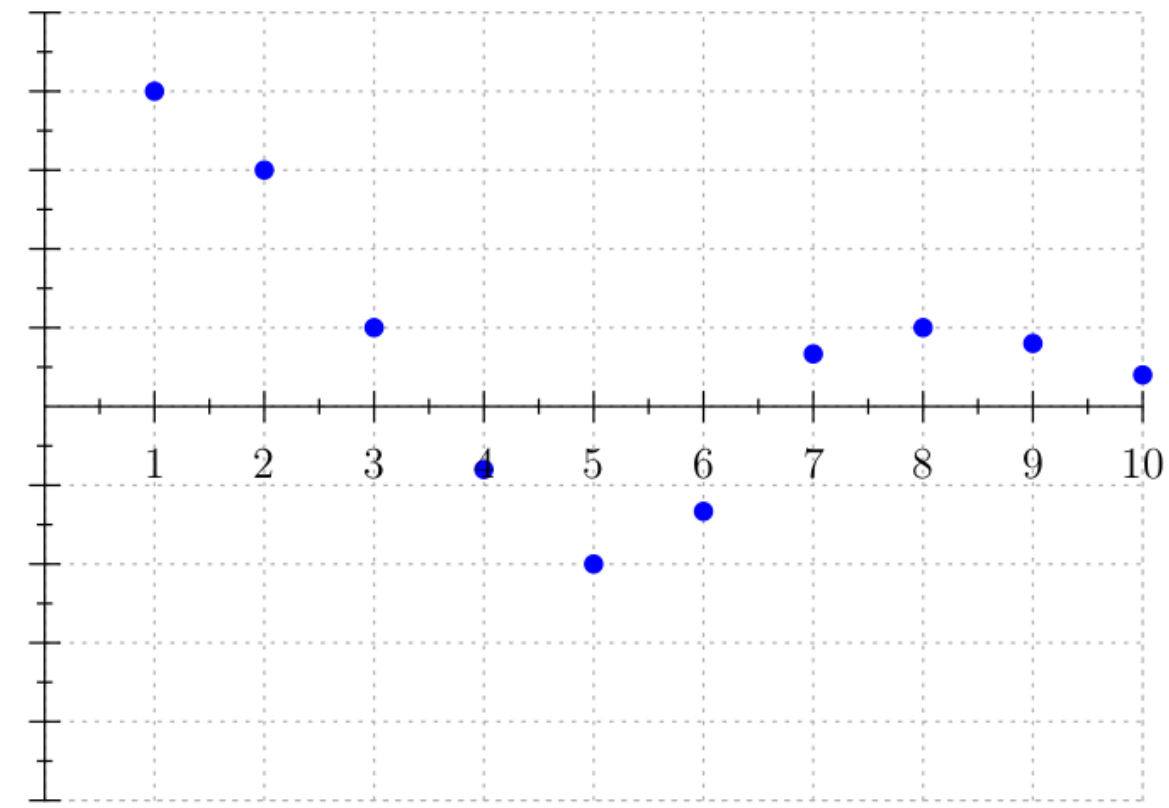

Figure 22: A damped oscillating sequence

A similar argument shows that the sequence $\left\{\text { inf } x_{n}(i, \infty)\right\}_{i}$ must either be a constant (some real number, or possibly negative infinity) or monotonically increasing. And so, in general, the sequence $\left\{\varepsilon_{i}\right\}$ will be monotonically decreasing, for any sequence ${ }^{17}$

As Dylan and Jay noted previously, showing that the sequence $\left\{\varepsilon_{i}\right\}$ was decreasing was not sufficient to show convergence of the sequence $\left\{x_{n}\right\}$. Dylan seemed to recall that something was missing, "Our definition of convergence to start off with was that this error...was decreasing? Right? And going to zero?" With this question they both consulted their definition of convergence, and

17 This is true except in the case where either the supremum or the infimum of the sequence is infinite. But in either of those cases the sequence is unbounded and therefore diverges. 
confirmed that they still needed to show that the sequence $\left\{\varepsilon_{i}\right\}$ actually decreased to zero.

There were a number of interesting features of their activity as they tried to prove this rigorously. Dylan and Jay argued about whether they could assume the existence of a least-upper bound for their sequence $\left\{x_{n}\right\}$. They then used their understanding of least-upper bounds as a tool-for completing the proof. Along the way they also wrestled with quantifiers; in some statements they were using $x_{i}$ to stand for a specific element of the sequence $\left\{x_{n}\right\}$, while in others they were using it to stand for all elements. Each of these will be elaborated below.

Dylan wrote out their definition of $\varepsilon_{i}$ for the hypothesized monotonic sequence, $\left\{x_{n}\right\}$ :

$$
\varepsilon_{i}=\left|L-x_{i}\right|,
$$

where $L$ represented the supremum of $\left\{x_{n}\right\}$ and " $x_{i}$ " was used in place of "inf $x_{n}(i$, $\infty)$ " since the sequence was monotonically increasing. But Jay was uncomfortable assuming that such an $L$ existed.

"My problem with using the sup, is that you assume that this- it's like you're assuming it converges...So if there's nothing bigger than it, and...I can always do whatever integer I want- I can always get as close as I want to it. So you're basically arguing that it converges."

In some sense Jay was correct. Assuming the existence of the supremum is the key step in completing the proof, and he had basically outlined the remainder of the argument. Dylan, though, felt differently: "But I think we can make an argument that the sup exists, from just knowing that there's some 
maximum bound ${ }^{18}$." His argument relied on his intuitive understanding of the real numbers, and in particular their representation via the real number line. He argued that, if the given upper bound $b$ was not the least upper bound, then there had to be a smaller upper bound, $c$. (Italics represent the student's emphasis.)

Jay: But we don't know that there's another-

Dylan: There has to be. That's what I'm saying. By the properties of the real numbers here. If there- if this $\left[x_{i+1}\right]$ never gets- like, if I pick a number that's smaller than $b$, that this value $\left[x_{i+1}\right]$ is never bigger than, then- I guess that's how a number line works, right? Let's say it does this kind of increasing behavior [Figure 23], but this is our $b$, so there is some value $c$. That's less than $b$. If this statement [upper bound inequality] is also true, if we can replace some $c$ for this $b$ ? And this inequality remains true? We keep doing that.

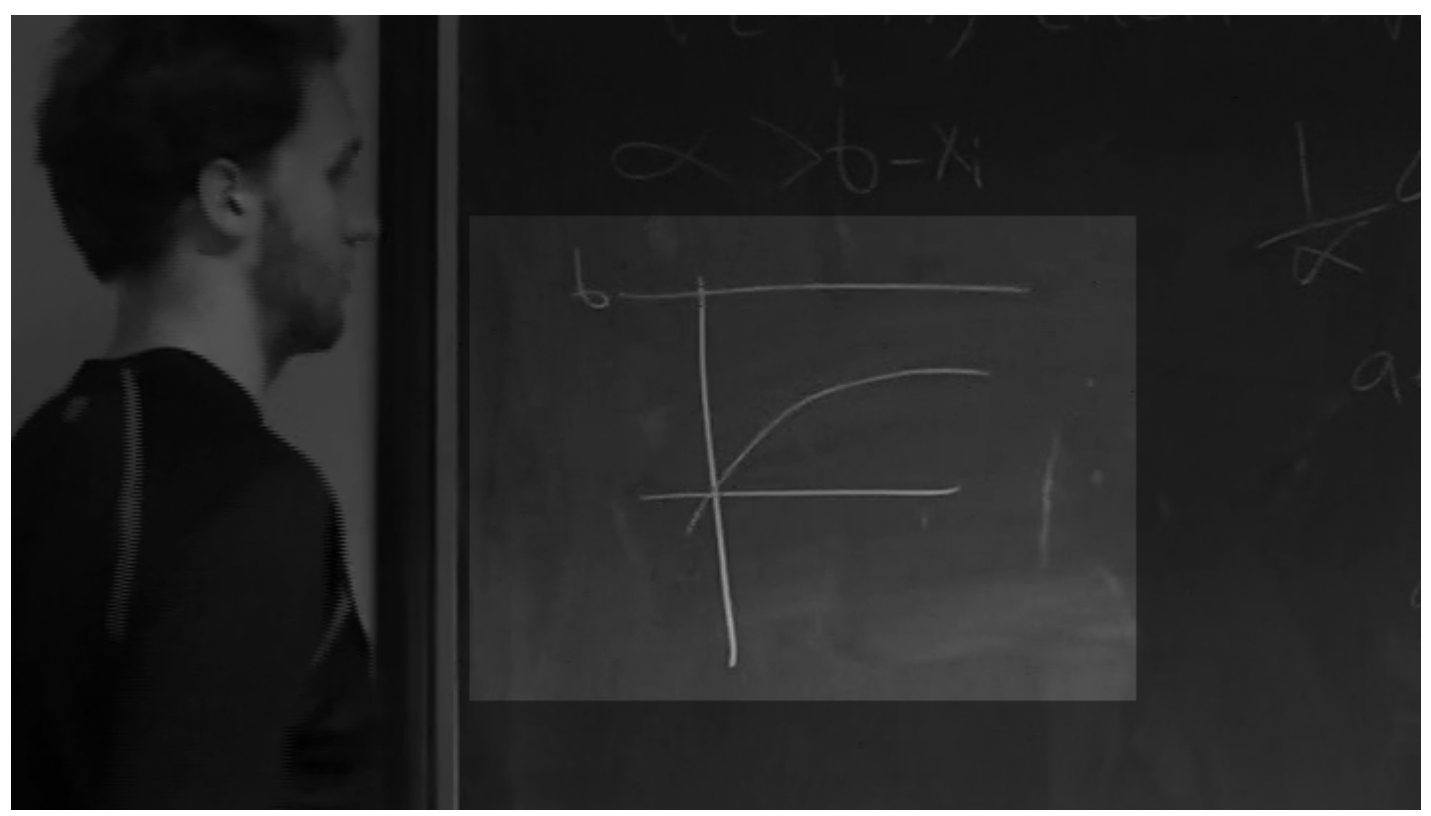

Figure 23: The sketch supporting Dylan's argument that they could assume the existence of a supremum.

18 Throughout this discussion Dylan used “maximum bound” in a way that was generally consistent with "upper bound". 
By appealing to "properties of the real numbers", and stating that "I guess that's how a number line works, right?" Dylan seemed to indicate that, for him, the existence of a supremum was a feature of the real numbers, something safe to assume without proof. Further, this argument convinced Jay that it was reasonable to use a supremum to continue with their proof.

Assuming the existence of a supremum, $L$, of their sequence $\left\{x_{n}\right\}$, all that remained was to show that the sequence $\{\varepsilon\}$ decreased to zero. Recall that, according to Dylan and Jay's definition, a sequence "decreased to zero" provided that for any positive real number, they could find elements of the sequence less than that number. Dylan chose an arbitrary, positive real number, $\alpha$, and set up the inequality that he and Jay attempted to verify:

$$
\alpha>\varepsilon_{i}=\left|L-X_{i}\right|
$$

They quickly rearranged the inequality, arriving at:

$$
x_{i}>L-\alpha,
$$

which they interpreted as: "We can always find an $x_{i}$ bigger?" If not, then the following inequality must be true:

$$
x_{i} \leq L-\alpha
$$

Dylan and Jay interpreted this to mean that $L-\alpha$ was an upper bound for the sequence, and an upper bound that was clearly smaller than $L$. But since $L$ was the least upper bound, this appeared to be a contradiction. Dylan and Jay then began to re-examine their argument to determine if this in fact completed the proof. 
It became apparent that there seemed to be some confusion about what $x_{i}$ stood for in each of the above inequalities. When examining the first inequality, Jay asked, "You're saying this $x_{i}$ is the entire sequence, right?" After a moment's consideration, Dylan responded, "Yeah. It's the entire sequence." Unfortunately this did not accurately reflect the argument that they had constructed.

When initially constructing the argument and writing the inequalities on the board, Dylan and Jay were verbally using quantifiers to make sense of these inequalities. However, they did not express these quantifiers in mathematical notation. In the first inequality, Dylan said "an $x_{i}$ ", indicating an existential quantifier. But then when considering the negation of that inequality, he said "every single element here" (pointing at the $x_{i}$ ), indicating a universal quantifier.

In the interest of time, I chose to re-voice their original argument, inscribing the proper quantifiers as I spoke.

"Let's be a little careful. So I think what we're saying here is, we're trying to find an index, right? So we're kind of like, there is an $i$ that does this [first inequality]. And if the answer's "No," then the negation of that is, "Then for all $i$," that [second inequality] has to be true."

Dylan and Jay affirmed my summary, and then proceeded to recapitulate the proof one last time. Jay summarized, "The two big implications were that there's a least-upper bound, and that least-upper bound implies convergence." Dylan agreed, and with that they had completed their proof of their Monotone Convergence Theorem. 


\section{Summary}

The design heuristic of emergent models is useful for describing the development of Dylan and Jay's thinking with respect to convergence and completeness. When they initially justified that their algorithm would find a root, their reasoning could be modeled by the completeness of the real numbers (specifically as characterized in the Monotone Convergence Theorem). In order to engage the students in vertical mathematizing, I tasked Dylan and Jay with reflecting on and trying to prove that justification as a conjecture. Least-upper bounds emerged as a record-of their thinking about conditions for a monotonic sequence to converge to an upper bound. Again, I codified this conjecture and tasked the students with analyzing and then proving it, supporting further refinement of this form of the global emergent model. Equipped with the language and concept of least-upper bounds, Dylan and Jay returned to the proof of the MCT. In order to complete this proof, Dylan and Jay had to convince themselves of the existence of least-upper bounds under the conditions of their theorem. They then had to use the properties of least-upper bounds, coupled with their definition of convergence, to construct a formal, algebraic argument to complete the proof of their MCT. The completeness of the real numbers in the form of the Least-Upper Bound Property ${ }^{19}$, had become a tool-for more formal reasoning about completeness and convergence.

With the proof completed, and with their newly constructed understandings

19 A non-empty set that is bounded above has a least-upper bound. 
of sequence convergence and completeness, I asked Dylan and Jay to once again return to their algorithm and the sequence of approximations it generated. I then tasked them with formally proving that their algorithm would find a root. Interestingly, rather than applying the Monotone Convergence Theorem directly, Dylan and Jay used the techniques from their proof. They first verified that their sequence of approximations was monotonically increasing, and that it was bounded above. By their previous argument, this allowed them to conclude that there was a least-upper bound for their approximations. In a similar proof by contradiction, they formally showed that their sequence of approximations must converge to that least-upper bound. This marked the end of the second phase of the instructional sequence, and the question of why such a number must be a root motivated the transition to the third and final phase of the instructional sequence. The rest of that story can be found in The Intermediate Value Theorem as a Starting Point for Inquiry-Oriented Advanced Calculus (Strand, in preparation).

\section{Discussion}

In the context of developing a proof of the Intermediate Value Theorem, we have seen how the informal strategies of two students anticipated formal characterizations of completeness. Two characterizations of completeness emerged in this teaching experiment, both of which were rooted in the specific 
tasks with which Dylan and Jay were engaged. The Monotone Convergence Theorem (MCT) emerged because the students were considering the convergence of their (monotonically increasing, bounded) sequence of approximations to a root. That such a sequence must converge was intuitively clear to them. The rest of their activity took place within the context of proving this idea. Least-upper bounds also emerged from their activity, for the first time when they were trying to identify what conditions would guarantee that a monotonically increasing sequence would converge to an upper bound. Dylan and Jay subsequently utilized this idea, using the term "max", to define an "error" term for sequences in general, with which they defined sequence convergence. Least-upper bounds also supported the students in constructing a formal definition of what it meant for a sequence to decrease to zero. Finally, in constructing a proof of the MCT, Dylan and Jay debated the existence of leastupper bounds, ultimately accepting their existence as a consequence of the real number line. The existence and properties of least-upper bounds were the key ideas in the ultimate completion of their proof of the MCT.

One way to frame this development is using the RME construct of emergent models. In justifying the convergence of their sequence of approximations, the students' thinking could be modeled by the larger concept of the completeness of the real numbers. Dylan and Jay were eventually able to use aspects of this model as a tool-for reasoning more formally about convergence. The development of this model was inextricably tied up with the 
development of their understanding of convergence. So much so, in fact, that it would be impossible to describe one without describing the other. It was in the process of defining sequence convergence that least-upper bounds emerged as a record-of their thinking. Through the process of reflecting on and formally defining "maximum" (which helped them solidify their definition of sequence convergence) completeness, specifically as signified with least-upper bounds, began to transition to a tool-for the students to reason more formally about convergence and to complete a proof of the MCT. In this way the development of their understanding of completeness supported and was supported by the development of their understanding of sequence convergence.

Future research will investigate further the nature of this interdependent concept development in this context. For example, in my first teaching experiment, the pair of students considered a sequence of nested, shrinking intervals, rather than a sequence of approximations to a root. This thinking could also be modeled by the larger concept of the completeness of the real numbers, as it is essentially the Nested Interval Property. Though I did not pursue the development of that model in that first teaching experiment, didactic phenomenology suggests that analyzing and codifying this as a conjecture, and working to develop a proof of said conjecture, would be a promising approach for supporting students in reinventing formal characterizations of sequence convergence and completeness. From an instructional design perspective, it will be important to investigate the constraints and affordances of going through this 
process starting with different characterizations of completeness. While this research suggests a promising instructional design approach, knowing more about how these two concepts develop together would be invaluable for these efforts. In particular it will be interesting to know if least-upper bounds serve the same role as a transformational record, and if that characterization is the one students choose as a foundation.

Also of great interest is students' perception of completeness from an axiomatic perspective. In this research Dylan and Jay seemed content to rely on the existence of least-upper bounds. In that sense the existence of least-upper bounds served as an axiom in the classical Greek sense: it was a property that seemed evidently true solely from the properties of the real numbers. But it is also true that I did not press Dylan and Jay to question this assumption. On what other characterizations of completeness might students rely when trying to prove the existence of least-upper bounds? I would be very interested to know how other students view the choice of a foundation; when is it okay to "stop digging"? Answers to these questions could have a strong impact on instruction of advanced calculus/real analysis.

This paper contributes to our understanding of how students think about completeness. Specifically in the context of the Intermediate Value Theorem, there is strong evidence that completeness can be a powerful model, first as a record-of student thinking about convergence, and then for use by the students as a tool-for developing more formal conceptions of sequence convergence and 
completeness itself. This further suggests that intuitive notions of completeness could support students in developing their understanding of sequence convergence and completeness in other contexts, as well; for example, in an IBL (Inquiry-Based Learning) or even a traditional lecture-based advanced calculus course. There is also evidence that these informal student characterizations of completeness are rooted in representations of the real numbers as a number line; the historical development of completeness lends credence to this idea (Dedekind, 1901). While there is still much to uncover about how students think about completeness and how that thinking might progress, it is evident that there are important connections between completeness and convergence in students' minds. 


\section{Paper \#3 - Pedagogical Inspirations for Advanced Calculus from Cauchy's Proof of the Intermediate Value Theorem}

Making the transition from more computation-based lower-division math classes to more abstract, proof-based upper-division courses can be quite challenging for undergraduate students. The transition from calculus to advanced calculus is a prime example. Part of this challenge lies in the aforementioned dramatic shift in student activity, from a dependence on algorithms and computational techniques to a focus on definitions, theorems, and proofs. In order to address these challenges, I sought to engage students in activities in a context that would engage their knowledge and skills gained through the calculus sequence, and would motivate their investigation into the deeper questions of the how and why of calculus. I wanted to give them a problem embedded in a context that would cause them to seek things like a formal definition of sequence convergence. In this article I will explain how Cauchy's proof of the Intermediate Value theorem provides just such a context.

The instructional sequence described in this paper comes from a larger instructional design project for advanced calculus, developed using the design heuristics of Realistic Mathematics Education (RME). Briefly, the RME approach is founded on the principle of guided reinvention: "The idea is to allow learners to come to regard the knowledge they acquire as their own private knowledge, knowledge for which they themselves are responsible" (Gravemeijer, 1999). Thus 
mathematical learning is conceptualized as a process by which "formal mathematics comes to the fore as a natural extension of the student's experiential reality" that is, an extension of the student's informal knowledge and intuition (Gravemeijer, 1999).

This instructional sequence is intended for use in a classroom centered around student inquiry. There are a number of powerful, well-regarded sets of IBL (Inquiry-Based Learning) notes for advanced calculus/real analysis (Mahavier \& Clark, 2016) that have been developed and refined through many, many classroom implementations. Such notes, generally speaking, are primarily geared toward supporting students in developing the deductive system of real analysis from the ground up; that is, students are presented with foundational definitions, and then tasked with proving theorems and conjectures, culminating with powerful results like the Fundamental Theorem of Calculus. The goal of this project, however, is to develop student-centered, research-based curriculum for advanced calculus that supports students in formalizing their informal knowledge. Another way to frame this is that, in the RME approach, we give students a problem in a context with which they are intuitively familiar (e.g., the IVT), and then the students build the machinery (definitions, theorems, etc.) needed to solve the problem. In any case, the underlying motivation is the same: to have the students doing the math in the classroom, rather than the teacher. The teacher should act only as an expert guide. 


\section{The Intermediate Value Theorem:}

$$
\text { If } f:[a, b] \rightarrow \mathbb{R} \text { is continuous and }
$$
$f(a) \neq f(b)$, then for every $y$ between $f(a)$ and $f(b)$ there exists a $c \in(a, b)$ so that $f(c)=y$.

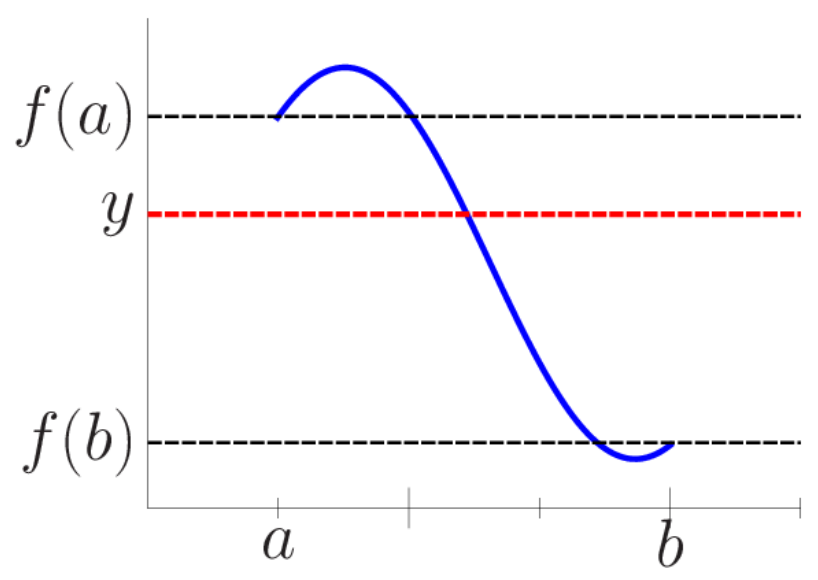

Figure 24: The Intermediate Value Theorem for continuous functions.

Cauchy's proof of the Intermediate Value Theorem (IVT) was significant in the historical development of real analysis for a number of reasons (Grabiner, 1981). He and Bolzano are credited with developing the first formal proofs of the IVT in the early 1820s. Up until that time, the IVT was generally taken to be an obvious consequence of continuity, if not a definition of continuity itself (Lützen, 2003). Cauchy's proof technique was novel as well: he adapted the approximation techniques of Lagrange and others into tools for proving existence (Grabiner, 1981). In the subsequent sections I will describe an instructional 
sequence, inspired by Cauchy's proof, that supports students in developing their own proof of the IVT. Along the way, students will discover the need for formal definitions of sequence convergence and continuity, in addition to a formal characterization of the completeness of the real numbers. These are some of the core concepts in advanced calculus, so developing formal conceptions in this way will give students a solid footing on which to continue their investigations of real numbers and functions.

\section{An Approximation Algorithm}

Students begin their investigations with a relatively simple question: Does $p(x)=x^{5}-x-5$ have a root in $[0,2]$ ? This polynomial is an unsolvable quintic, and so no analytic techniques will help them find the root. This might seem a funny way to start off investigations designed to build on students informal knowledge, but the goal here is to get students thinking in terms of the Intermediate Value Theorem (IVT). Of course this polynomial does have a root on the given interval, and though the exact wording may vary, student justifications for this amount to a conjecture that is equivalent to the IVT: namely that the polynomial is continuous and changes sign on the interval, so it therefore must cross the $x$-axis in that interval. It is the proof of this general conjecture, that a sign change of the (continuous) function implies a root, that drives the subsequent investigations. Trying to establish this intuitive results will motivate the students to develop the 
definitions and proof techniques necessary to do so.

In order to lay the foundation for this proof, we set the students a problem whose solution requires them to construct an algorithm that, in its fundamental features, resembles the algorithm used by Cauchy in his proof of the IVT. Given, for now, that their conditions imply the existence of a root, students engage with the following task: Approximate the root to exactly two decimal places. The students' earlier assertion that the root existed because the function changed sign provides a simple and powerful solution to this problem of approximation. Checking the sign of the function at values within the interval provides an expedient way to narrow their search for the root: a point in the interior of the interval must evaluate to either positive, negative, or zero. If zero, then the search for a root is completed. If not, then the root must lie between sign changes of the function, and we now have a smaller range to consider.

Furthermore, the distance between these successive values provides a bound for the error in using an interior point for our approximation, which allows us to know for certain how many digits of our approximation are correct. Followup tasks require the students to achieve greater degrees of accuracy: for example, Now approximate the root to six decimal places, and then, Approximate the root to an arbitrary degree of accuracy. This provides students with a need to iterate their reasoning into an approximation algorithm. 


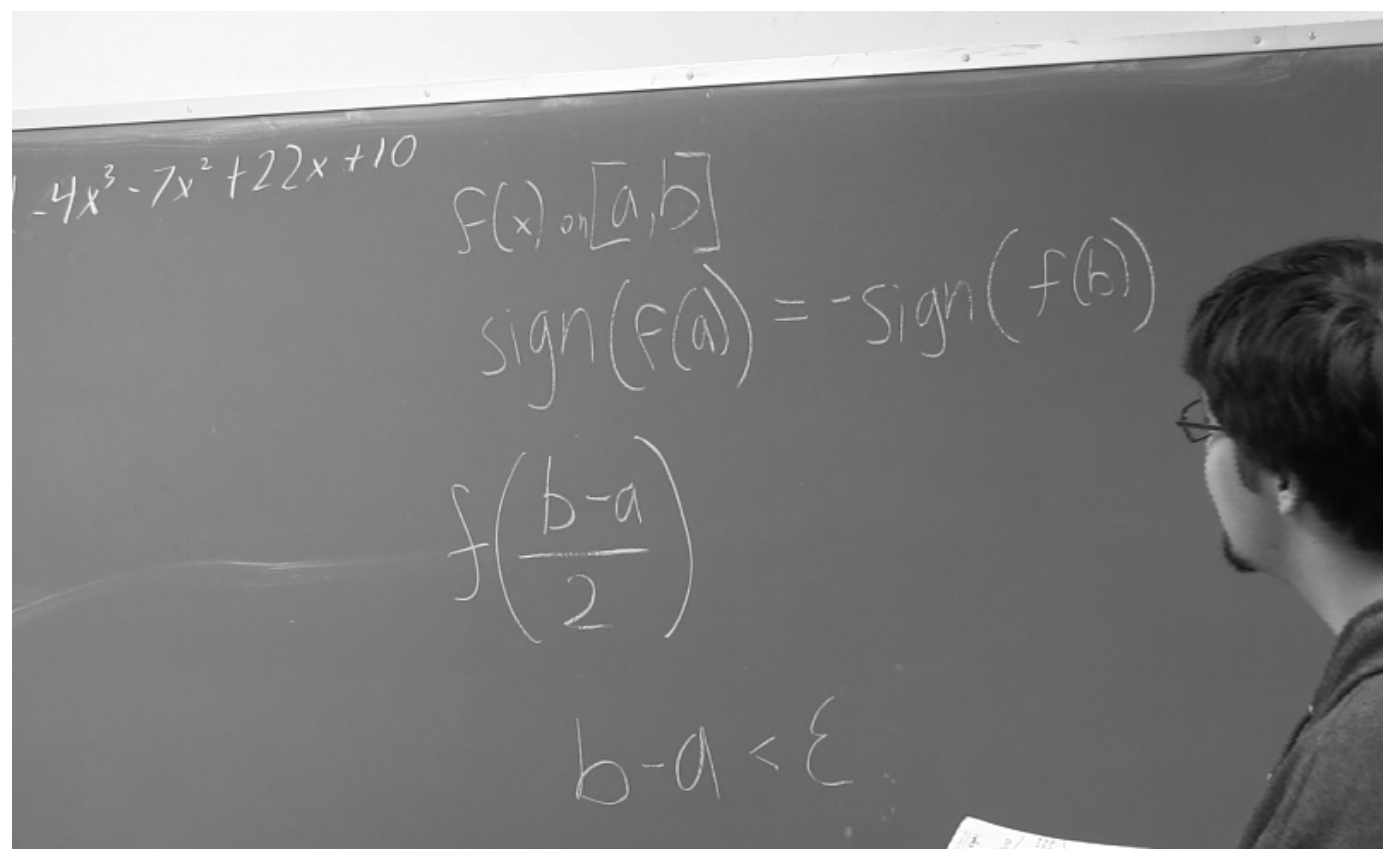

Figure 25: A student's brief statement of their algorithm using the Bisection Method.

This algorithm may take on different forms, but should utilize the same core feature: namely that of iteratively approaching a root by analyzing the sign change of the function. I have worked with two separate pairs of students investigating this context. In Figure 25, a student has described their algorithm, which was essentially equivalent to the Bisection Method. Another pair of students I worked with developed an algorithm that involved checking the sign at successive decimal values; when the sign changed, they then went to the next unknown decimal place and began checking again (Figure 26). This amounted to a decimal expansion of the root. Though markedly different, both of these algorithms used the sign change of the function to generate increasingly accurate 
approximations to a conjectured root, and both algorithms resulted in a number of convergent sequences.

First Iteration:

\begin{tabular}{|c|c|c|c|c|c|c|}
\hline $\boldsymbol{x}$ & 1.41 & 1.42 & 1.43 & 1.44 & 1.45 & 1.46 \\
\hline$+/$ & - & - & - & - & - & + \\
\hline
\end{tabular}

Second Iteration:

\begin{tabular}{|c|c|c|}
\hline $\boldsymbol{x}$ & 1.451 & 1.452 \\
\hline$+/-$ & - & + \\
\hline
\end{tabular}

Third Iteration:

\begin{tabular}{|c|c|c|c|c|c|c|c|c|c|c|}
\hline $\boldsymbol{x}$ & 1.4511 & 1.4512 & 1.4513 & 1.4514 & 1.4515 & 1.4516 & 1.4517 & 1.4518 & 1.4519 & 1.4520 \\
\hline$+/-$ & - & - & - & - & - & - & - & - & - & + \\
\hline
\end{tabular}

Figure 26: A few sample iterations of students' Decimal Expansion algorithm for approximating a root.

\section{Sequence Convergence \& Completeness}

After developing their approximation algorithm, the development of a proof of their conjectured IVT logically requires students to focus on the convergence of one (or more) of the sequences generated by their approximation algorithm. One question that can prompt these investigations is: How do you know that your algorithm will find a single, real number? Due to the nature of the problem, student justifications will amount to characterizations of the completeness of the real numbers. For example, a pair of students who developed an algorithm based 
on the Bisection Method argued its finding of a root using a justification equivalent to the Nested Interval Property. They recognized that the width of the interval in question was a bound on the error in choosing any point in that interval to approximate the root. For example, if they had narrowed the interval down to $[1.25,1.5]$, then the error in choosing any point in that interval as an approximation to the root could never be worse than $1.5-1.25=0.25$. Because each iteration of their algorithm halved the maximum possible error, in the limit the error would go to zero. The idea that a sequence of (nested) intervals whose length goes to zero contains a unique real number is essentially the Nested Interval Property.

For a pair of students who developed the Decimal Expansion algorithm, the justification was an entirely different characterization of the completeness property. With their implementation of the algorithm, they chose to focus only on the sequence of decimal approximations (e.g., 1.4, 1.41, 1.4.19,..., etc.). These students argued that this sequence of approximations must converge because it was increasing and bounded above (by the root, for one). This is essentially the Monotone Convergence Theorem (Figure 27). 


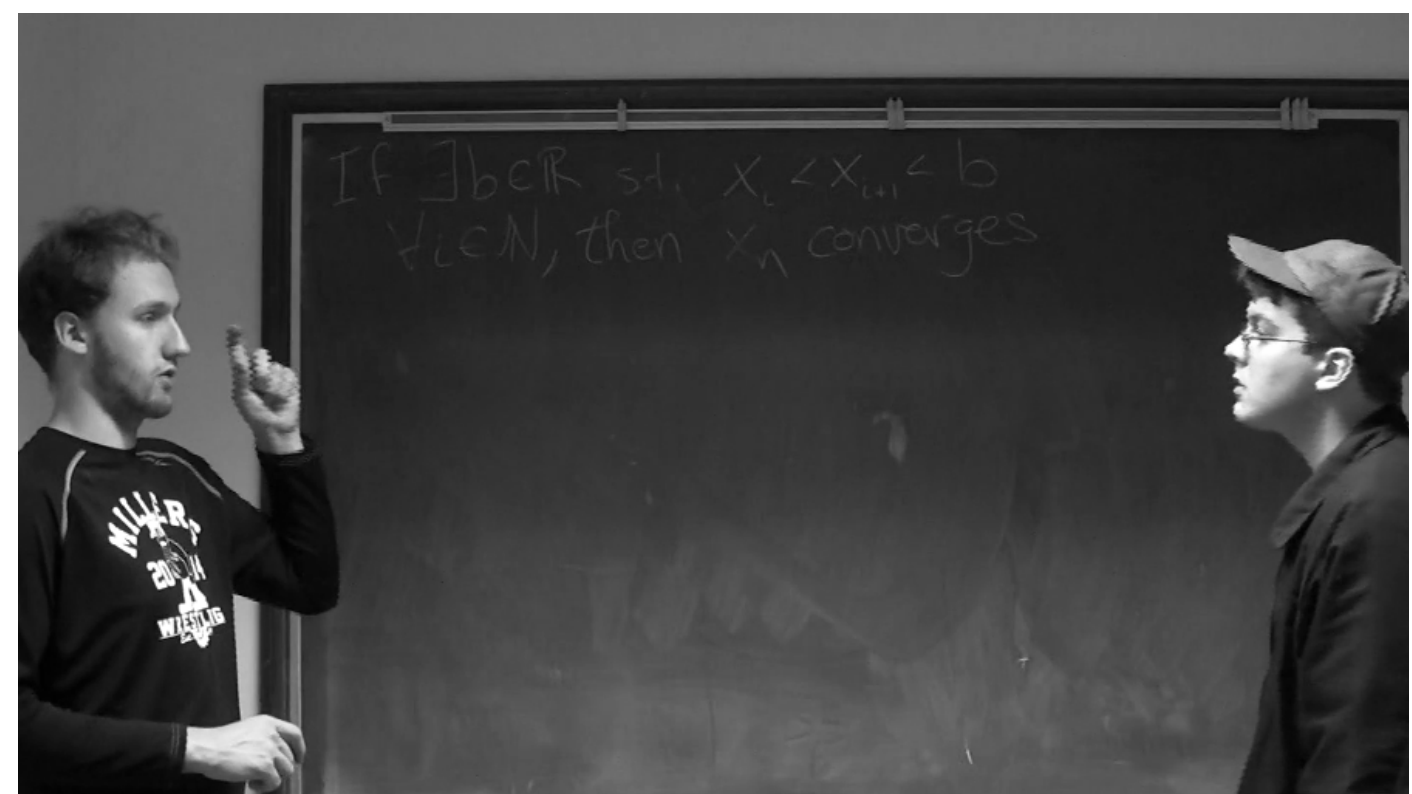

Figure 27: Students discussing how to prove their conjecture (which is essentially the $M C T)$.

Codifying their justification as a conjecture provides students the next problem to solve. This problem motivates the development of a formal definition of sequence convergence and of a formal characterization of completeness. The proof of any of these conjectures will require the students to appeal to another characterization of completeness, one which they can accept as a basic assumption without proof. This is due to the fact that completeness is an axiomatic property of the real numbers, since we are not constructing them from the Rational numbers. The students who argued using the MCT reinvented the existence of least-upper bounds as their formal characterization of completeness, and the idea of least-upper bounds was instrumental for them in completing the proof. Through the process of developing, analyzing, and refining this proof, 
students gain a more formal understanding of completeness.

In order to complete a formal proof, the students will also need to develop a formal definition of sequence convergence. Depending on the justification they chose, the students may only need a formal definition of sequence convergence that applies to a sequence monotonically decreasing to zero, or to a sequence monotonically increasing toward its limit. Subsequently, any of the myriad other sequences generated by their algorithm can be used to motivate more general definitions of sequence convergence. Though reflecting on their original algorithm and working to construct the proof should help students develop a formal definition, it has also been shown that generating a set of examples and non-examples can help support the process of developing a formal definition of sequence convergence (Swinyard \& Larsen, 2012; Oehrtman, Swinyard, \& Martin, 2014). With such a definition students should be ready to complete the proof of their characterization of completeness. In this way they have developed more formal understandings of sequence convergence and completeness, and formally established that their algorithm approximates something. All that remains is to formally prove the IVT (as they have conjectured it), which will involve repurposing their algorithm as a tool to establish the existence of a root candidate, and then to use continuity to show that it must, in fact, be a root. 


\section{Continuity}

With their approximation algorithm firmly established as a tool, and with their newly constructed understandings of sequence convergence and completeness, the students are now ready to return to the larger proof of the IVT. Building on their previous activity, they should be ready to begin proof construction: Prove that, under your conditions, a function $f$ must have a root in $[a, b]$. Students should be supported in re-purposing their algorithm, to use it to show the existence of a unique real number that serves as a root-candidate.

With a root-candidate in hand, the remainder of the proof involves using the continuity of the function to show that the root-candidate is a root of the function. Informal student conceptions are likely to take one (or both) of two forms: 1) a continuous function is one with no "jumps" or "breaks", or 2) the limit characterization from calculus: a function is continuous at a point if the limit at that point equals the function's value. In order to formalize the second characterization, students will need to develop a formal definition of the limit of a function at a point. This activity is supported by their previous work with their algorithm and with defining sequence convergence. 


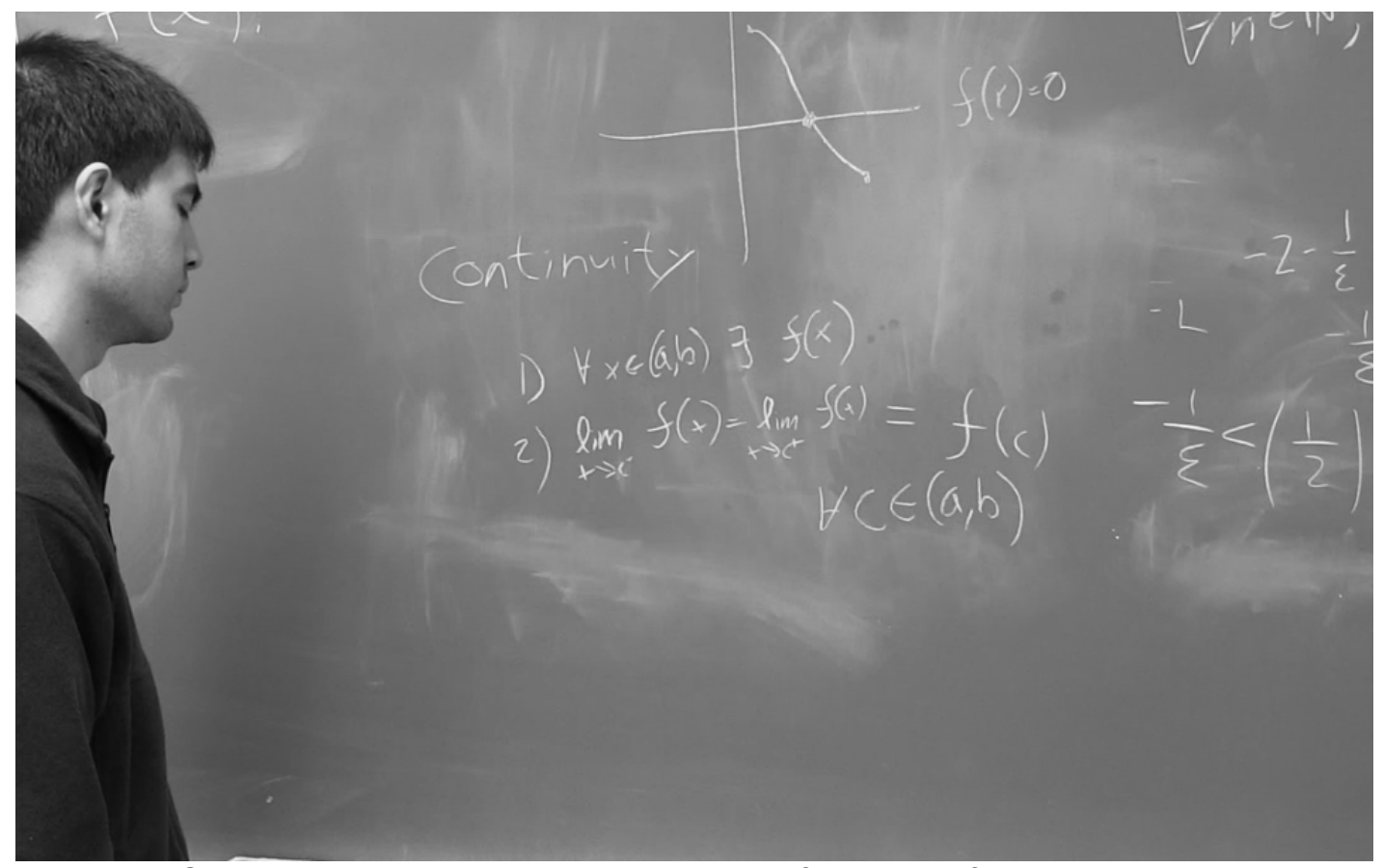

Figure 28: Students recall the limit characterization of continuity from calculus.

Formalizing the first characterization presents the opportunity of developing an epsilon-delta definition of continuity without first defining the limit of a function at a point. In this case it might be useful to have students formally define what is meant by a function having "no jumps or breaks". As a first attempt, one student I worked with suggested that the changes in $x$ and $y$ had to be proportional, somehow, so that a small change in $x$ could not result in a much larger change in $y$. While this statement is focused first on the inputs and lacks any usable quantification, this characterization bears some remarkable similarities to the reasoning behind the formal epsilon-delta definition of continuity. By codifying this statement, and having students analyze and refine it, 
students can be supported in developing a formal definition of continuity. However it is developed, with a formal definition of continuity students are ready to complete their proof of the IVT.

\section{Summary}

Cauchy's proof of the Intermediate Value Theorem offers a rich context for developing some of the fundamental concepts of real analysis. Further, it offers a very accessible entry point for students who have completed the calculus sequence. Student inquiry begins with approximating the root of a polynomial. Students should develop an algorithm that uses the sign change of the function to "zoom in" on the root. Student justifications for why this algorithm finds a root will depend on one (or more) of the sequences it generates, and these justifications will be equivalent to characterizations of the completeness of the real numbers (e.g., the Monotone Convergence Theorem, the Nested Interval Property, the Least-upper Bound Property, etc.). This can serve as a motivation to consider the development and proof of different characterizations of the completeness property. The proof of these characterizations will require students to develop a formal definition of sequence convergence. Finally, the task of putting together a proof of the Intermediate Value Theorem, building on the students' algorithm and their formal characterizations of sequence convergence and completeness, can be used to motivate the investigation of continuity, and the development of one or more formal definitions of continuity. In this way 
Cauchy's proof of the Intermediate Value theorem can be a touchstone that supports students in making the transition to the more formal world of advanced calculus, and specifically supports them in developing more formal conceptions of sequence convergence, completeness, and continuity.

While these insights have been presented for a classroom centered around student inquiry, I believe they could be useful in a more traditional classroom environment as well. The IVT is an intuitive result, and as such can be used as a starting point and a touchstone to motivate development of the topics of limits and convergence, the completeness of the real numbers, and what it means for a function to be continuous. Each of these topics can be thought of as branches, growing out of the trunk that is the IVT. When the development of one branch is complete, one can return to Cauchy's proof to motivate development along a subsequent branch. In this way some of the core ideas of advanced calculus/real analysis can be developed, and motivated by the historical development of analysis. 


\section{References}

Bartle, R. G., \& Sherbert, D. R. (2000). Introduction to real analysis, JohnWiley\&Sons. Inc., New York.

Bezuidenhout, J. (2001). Limits and continuity: some conceptions of first-year students. International Journal of Mathematical Education in Science and Technology, 32(4), 487-500.

Cornu, B. (1991). Limits. In D. Tall (Ed.), Advanced Mathematical Thinking (pp. 153-166). Dordrecht: Springer Netherlands.

Cobb, P., Confrey, J., diSessa, A., Lehrer, R., \& Schauble, L. (2003). Design experiments in educational research. Educational Researcher, 32(1), 9-13. doi:10.3102/0013189X032001009

Cottrill, J., Dubinsky, E., Nichols, D., Schwingendorf, K., Thomas, K., \& Vidakovic, D. (1996). Understanding the limit concept: Beginning with a coordinated process scheme. The Journal of Mathematical Behavior, 15(2), 167-192.

Davis, R. B., \& Vinner, S. (1986). The notion of limit: Some seemingly unavoidable misconception stages. The Journal of Mathematical Behavior, 5(3), 281-303.

Dedekind, R. (1901). Essays in the theory of numbers, 1. Continuity and irrational numbers, 2. The nature and meaning of numbers. Authorized translation by Wooster Woodruff Beman. Chicago Open Court Pub. Co. Retrieved from http://archive.org/details/essaysintheoryof00dedeuoft

Grabiner, J. V. (1981). The origins of cauchy's rigorous calculus. Cambridge, Mass.: MIT Press.

Gravemeijer, K. (1998). Developmental research as a research method. In Mathematics education as a research domain: A search for identity (pp. 277-295). Springer Netherlands.

Gravemeijer, K. (1999). How emergent models may foster the constitution of formal mathematics. Mathematical Thinking and Learning, 1(2), 155-177. http://doi.org/10.1207/s15327833mtl0102 4

Gravemeijer, K. (2004). Local instruction theories as means of support for teachers in reform mathematics education. Mathematical Thinking and Learning, 6(2), 105-128. http://doi.org/10.1207/s15327833mtl0602_3

Jayakody, G., \& Zazkis, R. (2015). Continuous problem of function continuity. For the Learning of Mathematics, 35(1), 8-17.

Johnson, E. (2014). Two metaphors for realistic mathematics education design heuristics: implications for documenting student learning. Seventeenth 
Special Interest Group of the Mathematical Association of America on Research in Undergraduate Mathematics Education Conference on Research in Undergraduate Mathematics Education. Denver, CO.

Krantz, S. G. (2013). Real analysis and foundations. CRC press.

Larsen, S. (2004). Supporting the Guided Reinvention of the Concepts of Group and Isomorphism: A Developmental Research Project. Unpublished Dissertation, Arizona State University.

Larsen, S. (2013). A local instructional theory for the guided reinvention of the group and isomorphism concepts. The Journal of Mathematical Behavior, 32(4), 712-725. doi:10.1016/j.jmathb.2013.04.006

Larsen, S., \& Lockwood, E. (2013). A local instructional theory for the guided reinvention of the quotient group concept. The Journal of Mathematical Behavior, 32(4), 726-742. doi:10.1016/j.jmathb.2013.02.010

Lützen, J. (2003). The foundations of analysis in the 19th century. In H. N. Jahnke (Ed.), A history of analysis (Vol. 24, pp. 155-191). American Mathematical Society.

Mahavier, T., \& Clark, D. (Eds.). (2016). Journal of Inquiry-Based Learning in Mathematics. Retrieved from http://www.jiblm.org/guides/index.php? category=jiblmjournal

Moru, E. K. (2009). Epistemological obstacles in coming to understand the limit of a function at undergraduate level: A case from the national university of Lesotho. International Journal of Science and Mathematics Education, 7(3), 431-454.

Oehrtman, M. (2009). Collapsing dimensions, physical limitation, and other student metaphors for limit concepts. Journal for Research in Mathematics Education, 40(4), 396-426.

Oehrtman, M., Swinyard, C., \& Martin, J. (2014). Problems and solutions in students' reinvention of a definition for sequence convergence. The Journal of Mathematical Behavior, 33, 131-148.

Rasmussen, C., \& Kwon, O. N. (2007). An inquiry-oriented approach to undergraduate mathematics. The Journal of Mathematical Behavior, 26(3), 189-194. http://doi.org/10.1016/j.jmathb.2007.10.001

Rasmussen, C., \& Marrongelle, K. (2006). Pedagogical content tools: integrating student reasoning and mathematics instruction. Journal for Research in Mathematics Education, 37(5), 388-420.

Ross, K. A. (1980). Elementary analysis. New York: Springer-Verlag.

Rudin, W. (1964). Principles of mathematical analysis. McGraw-Hill New York. Sierpińska, A. (1987). Humanities students and epistemological obstacles related 
to limits. Educational Studies in Mathematics, 18(4), 371-397.

Stewart, J. (2003). Calculus: Early Transcendentals (5th ed.). Belmont, CA: Brooks/Cole.

Strand, S. R. (in preparation). Completeness and sequence convergence: Interdependent development in the context of proving the intermediate value theorem.

Swinyard, C., \& Larsen, S. (2012). Coming to understand the formal definition of limit: Insights gained from engaging students in reinvention. Journal for Research in Mathematics Education, 43(4), 465-493.

Szydlik, J. E. (2000). Mathematical beliefs and conceptual understanding of the limit of a function. Journal for Research in Mathematics Education, 31(3), 258-276.

Tall, D., \& Schwarzenberger, R. L. E. (1978). Conflicts in the learning of real numbers and limits. Mathematics Teaching, Vol.82, 44-49.

Tall, D., \& Vinner, S. (1981). Concept image and concept definition in mathematics with particular reference to limits and continuity. Educational Studies in Mathematics, 12(2), 151-169.

Wade, W. R. (2004). An introduction to analysis. Prentice Hall.

Wawro, M., Rasmussen, C., Zandieh, M., Sweeney, G. F., \& Larson, C. (2012). An Inquiry-oriented approach to span and linear independence: The Case of the magic carpet ride sequence. PRIMUS, 22(8), 577-599. http://doi.org/10.1080/10511970.2012.667516

Williams, S. R. (1991). Models of limit held by college calculus students. Journal for Research in Mathematics Education, 22(3), 219-236.

Zandieh, M., \& Rasmussen, C. (2010). Defining as a mathematical activity: A framework for characterizing progress from informal to more formal ways of reasoning. The Journal of Mathematical Behavior, 29(2), 57-75. 


\section{Appendix A}

List of Relevant Mathematical Definitions and Theorems

\section{Cauchy Sequence}

A sequence of real numbers $\{a n\}$ is called a cauchy sequence provided that for any $\varepsilon$ greater than 0 there exists an $N$ in the Naturals so that for any $m>=n>N$, $\left|a_{m}-a_{n}\right|<\varepsilon$.

\section{Dedekind Completeness}

Let $A$ and $B$ be sets of real numbers such that:

1. Every real number is either in $A$ or in $B$;

2. No real number is in $A$ and in $B$;

3. Neither $A$ nor $B$ is vacuous;

4. If $\alpha$ is in $A$ and $\beta$ is in $B$ then $\alpha<\beta$.

Then there is one (and only one) real number $\gamma$ such that $\alpha \leq y$ for all $\alpha$ in $A$, and $\gamma \leq \beta$ for all $\beta$ in $B$. (Rudin, 1953)

\section{The Intermediate Value Theorem (Bolzano's Theorem)}

Suppose that $f$ is continuous on $[a, b]$ and that $\operatorname{sign}(f(a))=-\operatorname{sign}(f(b))$. Then there exists a $c$ in $(a, b)$ so that $f(c)=0$.

\section{The Least-Upper Bound Property}

A non-empty, bounded set of real numbers has a least upper bound.

\section{The Limit of a Function at a Point}

$\lim _{x \rightarrow a} f(x)=L$ provided that for any $\varepsilon$ greater than zero there exists a $\delta$ greater than zero such that if $|x-a|<\delta$ then $|f(x)-f(a)|<\varepsilon$.

\section{The Monotone Convergence Theorem}

If a sequence $\left\{a_{n}\right\}$ is monotonic and bounded (i.e. there exists an $M$ so that $|a n|<$ $M$ for all $n$ ), then the sequence converges.

\section{The Nested Interval Property}

The intersection of any sequence of closed, bounded, nested intervals is nonempty. 


\section{Appendix B}

\section{Sample Data Analysis}

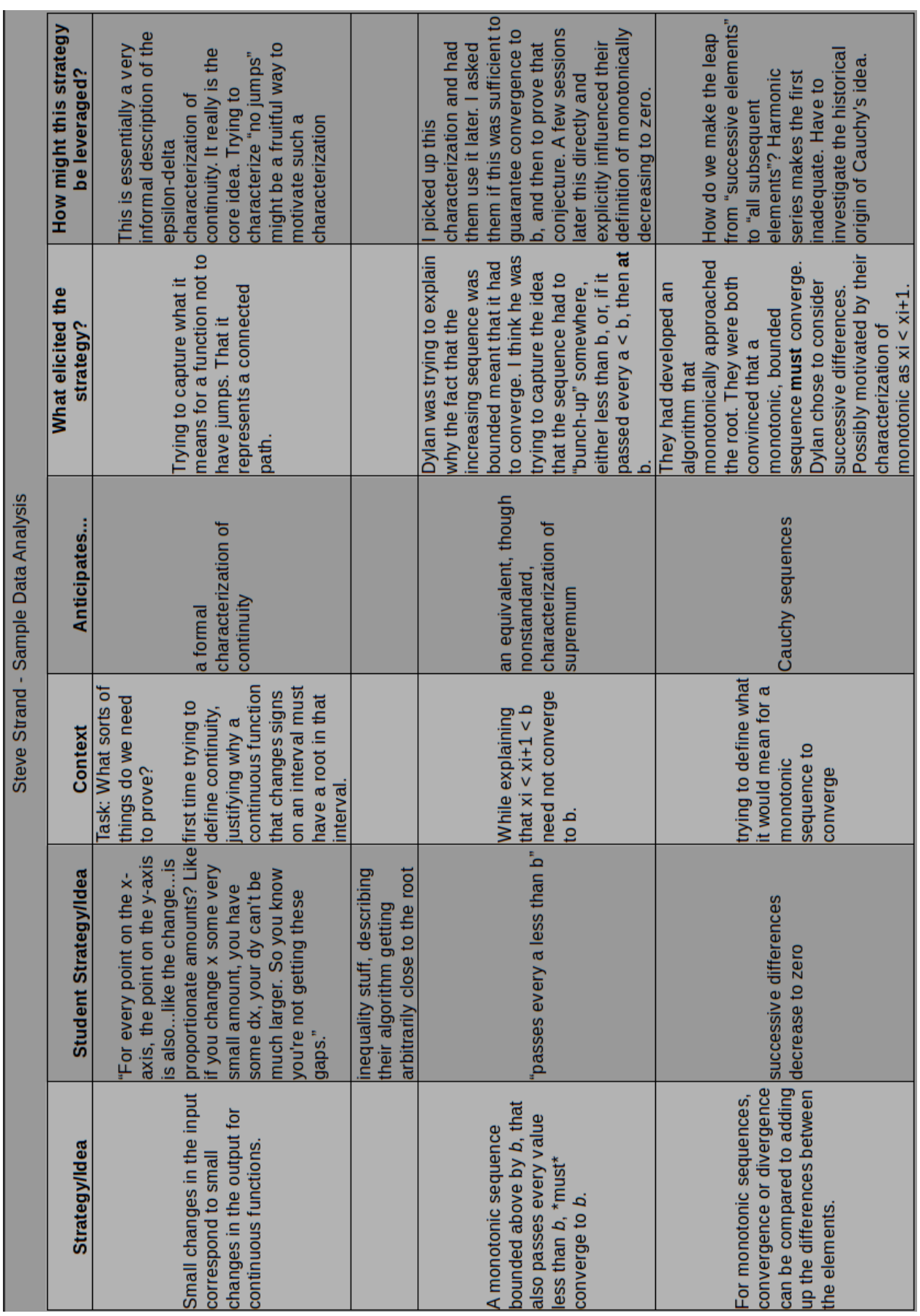

